\title{
SERRE'S TENSOR CONSTRUCTION AND MODULI OF ABELIAN SCHEMES
}

\author{
ZAVOSH AMIR-KHOSRAVI
}

\begin{abstract}
Given a polarized abelian scheme with action by a ring, and a projective finitely presented module over that ring, Serre's tensor construction produces a new abelian scheme. We show that to equip these abelian schemes with polarizations it's enough to equip the projective modules with non-degenerate positive-definite hermitian forms. As an application, we relate certain moduli spaces of principally polarized abelian schemes with action by the ring of integers of a CM field. More specifically, we consider integral models of zero-dimensional Shimura varieties associated to compact unitary groups. We show that all abelian schemes in such moduli spaces are, étale locally over their base schemes, Serre constructions of CM abelian schemes with positive-definite hermitian modules. We also describe the morphisms between such objects in terms of morphisms between the constituent data, and formulate these relations as an isomorphism of algebraic stacks.
\end{abstract}

\section{INTRODUCTION}

Let $R$ be a ring, possibly non-commutative, and free of finite rank over $\mathbb{Z}$. Let $(A, \iota)$ be an abelian scheme $A$ over a base $S$, with an injective ring homomorphism $\iota: R \hookrightarrow \operatorname{End}_{S}(A)$ giving an $R$-action on $A$. Take $M$ to be a projective finitely presented right $R$-module. Serre's tensor construction associates to this data a new abelian scheme $M \otimes_{R} A$ over $S$, which is characterized by its functor of points $\mathrm{Sch}_{/ S} \rightarrow \mathrm{Ab}, T \mapsto M \otimes_{R} A(T)$ (Definition 1). The map $A \mapsto M \otimes_{R} A$ is functorial in $A$ and $M$, and preserves many desirable properties of $A$. This suggests the possibility of using it to relate families of abelian schemes. In order to do this, we first need to equip $M \otimes_{R} A$ with extra structures, in particular a polarization [20, 6] that is compatible with the $R$-action in the following sense.

Assume $R$ is equipped with a positive involution $r \mapsto r^{*}$ (Definition 44). Then the pair $(A, \iota)$ has a dual $\left(A^{\vee}, \iota^{\vee}\right)$, where $A^{\vee}$ is the dual abelian scheme of $A$, and $\iota^{\vee}(r)=\iota\left(r^{*}\right)^{\vee}$, for $r \in R$. A polarization $\lambda: A \rightarrow A^{\vee}$ is said to be $R$-linear if $\lambda \circ \iota(r)=\iota^{\vee}(r) \circ \lambda$.

Assume $M_{\mathbb{Q}}$ is free over $R_{\mathbb{Q}}$. The dual module $M^{\vee}=\operatorname{Hom}_{R}(M, R)$ has a natural right $R$-module structure, with $r \in R$ acting on $f \in M^{\vee}$ by $(f \cdot r)(m)=r^{*} f(m)$. Then $R$-linear maps $h: M \rightarrow M^{\vee}$ may be identified with sesquilinear forms $H: M \times M \rightarrow R$ via $H\left(m, m^{\prime}\right)=h(m)\left(m^{\prime}\right)$. Such a map $h$ is called hermitian if $H\left(m, m^{\prime}\right)=H\left(m^{\prime}, m\right)^{*}$, and non-degenerate if it's an isomorphism. Since $M_{\mathbb{Q}} \simeq R_{\mathbb{Q}}^{n}$, we may identify $h$ with an element of $H_{n}\left(R_{\mathbb{Q}}\right)$, the set of $n \times n$ hermitian matrices with entries in $R_{\mathbb{Q}}$. Then $H_{n}\left(R_{\mathbb{Q}}\right) \otimes \mathbb{R}$ is a formally real Jordan algebra (Definition $\mathbb{8}$ ) over $\mathbb{R}$. We say $h$ is positive-definite if its image under $H_{n}\left(R_{\mathbb{Q}}\right) \subset H_{n}\left(R_{\mathbb{Q}}\right) \otimes \mathbb{R}$ is positive (Definition [15). This notion does not depend on the choice of isomorphism $M_{\mathbb{Q}} \simeq R_{\mathbb{Q}}^{n}$ (Lemma 16).

Theorem A. Suppose $(A, \iota, \lambda)$ consists of an abelian scheme $A / S$, an $R$-action $\iota: R \hookrightarrow \operatorname{End}(A)$, and $R$-linear polarization $\lambda: A \rightarrow A^{\vee}$. Let $h: M \rightarrow M^{\vee}$ be R-linear. The map $h \otimes \lambda: M \otimes_{R} A \rightarrow$ $M^{\vee} \otimes_{R} A^{\vee}$ is a polarization on $M \otimes_{R} A$ if and only if $h$ is a positive definite $R$-valued hermitian form.

The above is Theorem 17 in the main text. That the abelian scheme dual to $M \otimes_{R} A$ is $M^{\vee} \otimes_{R} A^{\vee}$ is proved in Proposition 5. A special case of the theorem is due to Serre [16, appx], where $A$ is a specific elliptic curve in characteristic $p$. We also show that under some extra assumptions on $A$,

Department of Mathematics, California Institute of Technology, Pasadena, California

E-mail address: zavosh@caltech.edu. 
if $\lambda$ is principal, then $h \otimes \lambda$ is principal if and only if $h$ is non-degenerate (Proposition 18). For instance it's enough to assume $\operatorname{End}_{S}(A)$ is free over $R$.

We then apply the above result to the following moduli problem. Let $K$ be a CM-field of degree $2 g$ over $\mathbb{Q}, \Phi$ a CM-type for $K$, and $n$ a positive integer. Let $L \subset \mathbb{C}$ be the reflex field of $(K, \Phi)$. To every locally noetherian scheme $S$ over $\operatorname{Spec} \mathcal{O}_{L}$ we associate the category $\mathcal{M}_{\Phi}^{n}(S)$ of triples $(A, \iota, \lambda)$ consisting of an abelian scheme $A$ of relative dimension $n g$ over $S$, an injective $\mathcal{O}_{K}$-action $\iota$, and an $\mathcal{O}_{K}$-linear principal polarization $\lambda$. We also require that $(A, \iota, \lambda)$ satisfy the ideal condition $J_{\Phi} \operatorname{Lie}_{S}(A)=0$, where $J_{\Phi}$ is the kernel of

$$
\mathcal{O}_{K} \otimes \mathcal{O}_{L} \rightarrow \prod_{\phi \in \Phi} \mathbb{C}^{(\phi)}, \quad(\alpha \otimes \beta) \mapsto(\phi(\alpha) \cdot \beta)_{\phi} .
$$

Morphisms of $\mathcal{M}_{\Phi}^{n}(S)$ are $\mathcal{O}_{K}$-linear isomorphisms of abelian schemes that preserve the polarizations (see Definition 30).

The ideal condition is a refinement of the signature condition. That says for $a \in \mathcal{O}_{K}$, the characteristic polynomial of the induced action of $\iota(a)$ on $\operatorname{Lie}_{S}(A)$ should equal

$$
\prod_{\phi \in \Phi}(T-\phi(a))^{n} \in \mathcal{O}_{L}[T]
$$

viewed as a section of $\mathcal{O}_{S}[T]$ using the structure morphism $\mathcal{O}_{L} \rightarrow \mathcal{O}_{S}$. The ideal condition implies the signature condition (Corollary 33). If $n=1$ or the base $S$ has characteristic zero, the signature condition also implies the ideal condition. The ideal condition ensures that $\mathcal{M}_{\Phi}^{n}$ is proper and smooth of relative dimension 0 over $\mathcal{O}_{L}$ (Theorem [35). When either $n=1$ or $K$ is quadratic imaginary, this is a theorem of $\mathrm{B}$. Howard [10, 11]. The ideal condition $J_{\Phi} \operatorname{Lie}_{S}(A)=0$ allows us to generalize Howard's proof to all CM fields $K$.

We note that $\mathcal{M}_{\Phi}^{1}$ is the moduli stack of abelian schemes with CM by $\mathcal{O}_{K}$ of type $\Phi$. The stacks $\mathcal{M}_{\Phi}^{n}$ are zero-dimensional versions of moduli spaces considered by S. Kudla and M. Rapoport ([12], [13]), which are integral models of Shimura varieties of unitary type.

Using Theorem A, we can apply Serre's construction to the problem of constructing objects in $\mathcal{M}_{\Phi}^{n}$. Let $\operatorname{Herm}_{n}\left(\mathcal{O}_{K}\right)$ denote the category of pairs $(M, h)$ consisting of projective finitely presented $\mathcal{O}_{K}$-modules $M$ of rank $n$, equipped with a positive-definite non-degenerate $\mathcal{O}_{K}$-hermitian structure $h: M \rightarrow M^{\vee}$. Then for $(M, h) \in \operatorname{Herm}_{n}\left(\mathcal{O}_{K}\right)$ and $(A, \iota, \lambda) \in \mathcal{M}_{\Phi}^{1}$, we construct the object

$$
(M, h) \otimes(A, \iota, \lambda)=\left(M \otimes_{R} A, \mathbb{1}_{M} \otimes \iota, h \otimes \lambda\right) \in \mathcal{M}_{\Phi}^{n} .
$$

To describe all such objects in $\mathcal{M}_{\Phi}^{n}$ we define a tensor product of categories, by explicit generators and relations (Definition 21). Then we construct the groupoid $\operatorname{Herm}_{n}\left(\mathcal{O}_{K}\right) \otimes_{\mathrm{Herm}_{1}\left(\mathcal{O}_{K}\right)} \mathcal{M}_{\Phi}^{1}(S)$ for each $S$. This is carried out in $\S 2$. We suggest the reader skip details of the otherwise intuitive abstract constructions in $\S 2$, and consult the section as needed. Aside from the definitions the main result in $\S 2$ is Proposition 24, which by a combinatorial argument gives a concise presentation of a general morphism in the tensor product groupoid.

Serre's construction then induces a functor

$$
\Sigma_{S}: \operatorname{Herm}_{n}\left(\mathcal{O}_{K}\right) \otimes_{\operatorname{Herm}_{1}\left(\mathcal{O}_{K}\right)} \mathcal{M}_{\Phi}^{1}(S) \rightarrow \mathcal{M}_{\Phi}^{n}(S)
$$

For $\Sigma_{S}$ to have any significance, $\mathcal{M}^{1}(S)$ must be non-empty. We can assume this is the case after replacing $L$ by some finite extension, as long as $\mathcal{M}_{\Phi}^{1}(\mathbb{C}) \neq \emptyset$. We show $\mathcal{M}_{\Phi}^{1}(\mathbb{C}) \neq \emptyset$ if $K / F$ is ramified at any finite prime (Theorem 46$)$. Also if $\mathcal{M}_{\Phi}^{n}(\mathbb{C}) \neq \emptyset$ and $n$ is odd, we have $\mathcal{M}_{\Phi}^{1}(\mathbb{C}) \neq \emptyset$ (Proposition 47). In general, we assume $\mathcal{M}_{\Phi}^{1}(\mathbb{C}) \neq \emptyset$.

The functor $S \mapsto \operatorname{Herm}_{n}\left(\mathcal{O}_{K}\right) \otimes_{\operatorname{Herm}_{1}\left(\mathcal{O}_{K}\right)} \mathcal{M}_{\Phi}^{1}(S)$ defines a separated presheaf on the big étale site over $\operatorname{Spec} \mathcal{O}_{L}$. Letting $\operatorname{Herm}_{n}\left(\mathcal{O}_{K}\right) \otimes \mathcal{M}_{\Phi}^{1}$ denote the associated stack, Serre's construction induces a morphism

$$
\Sigma: \operatorname{Herm}_{n}\left(\mathcal{O}_{K}\right) \otimes \mathcal{M}_{\Phi}^{1} \rightarrow \mathcal{M}_{\Phi}^{n}
$$


Theorem B. Assume that $\mathcal{M}_{\Phi}^{1}(\mathbb{C}) \neq \emptyset$. Then:

(1) $\Sigma$ is an isomorphism of $\mathcal{O}_{L}$-stacks.

(2) If $S=\operatorname{Spec}(k)$, for $k$ an algebraically closed field, then $\Sigma_{S}$ is an equivalence of categories.

(3) If $S$ is locally noetherian over Spec $\mathcal{O}_{L}$, each object of $\mathcal{M}_{\Phi}^{n}(S)$ is étale locally on $S$ in the image of $\Sigma_{S}$ from (0.2).

The above is Theorem 52 in the paper. The proof begins by showing the functor $\Sigma_{S}$ is fully faithful (Proposition 36). This is done by comparing general forms of morphisms in $\mathcal{M}_{\Phi}^{n}$ and the tensor product. Essential surjectivity of $\Sigma$ is proved on the stalks of geometric points, first in characteristic zero by explicit construction, then extended to characteristic $p$ by smoothness of $\mathcal{M}_{\Phi}^{n}$ over $\operatorname{Spec} \mathcal{O}_{L}$.

For a restatement of the content of Theorem $B$ without the language of higher categories see Theorem 53 in the text.

Acknowledgements. Much of the content in this article is part of the author's thesis, written at the University of Toronto under the supervision of Stephen S. Kudla. I thank Professor Kudla for suggesting the problem and for subsequent encouragements. I thank Brian Conrad and Florian Herzig for looking over an early draft, and offering helpful comments and suggestions.

I also thank Ben Howard, who read the first version carefully, spotting mistakes and suggesting improvements. In particular the ideal condition $J_{\Phi} \operatorname{Lie}_{S}(A)=0$ in the definition of $\mathcal{M}_{\Phi}^{n}$ was suggested by him. I express gratitude to the anonymous referee for patiently reading the article, uncovering many mistakes, and suggesting significant improvements and simplifications, especially in the proofs of the final sections.

\section{Contents}

Introduction

Acknowledgements

1. Serre's Construction

1.1. Homomorphisms

1.2. Lie algebra and Tate module.

1.3. The dual abelian scheme

1.4. Polarizations

2. Tensor Product of Categories

2.1. Definitions

2.2. Tensor product over a 2-group

3. Application to Moduli Spaces of Abelian Schemes.

3.1. The moduli space $\mathcal{M}_{\Phi}^{n}$

3.2. Serre construction of $\mathcal{M}_{\Phi}^{n}$ : the morphisms

3.3. Serre construction of $\mathcal{M}_{\Phi}^{n}$ : the objects

3.4. Stackification

3.5. A simple example

Final Remarks

References

\section{Serre's Construction}

In this section we recall the Serre tensor construction, then establish some basic properties of the abelian schemes arising from it, such as the possible homomorphisms between them, their Tate modules, Lie algebras, and their dual abelian schemes. We then study the polarizations on such abelian schemes. 
Let $R$ be a ring, possibly non-commutative, and free of finite rank over $\mathbb{Z}$. An abelian scheme with an $R$-action is a pair $(A, \iota)$, where $A$ is an abelian scheme over some base $S$, and $\iota: R \rightarrow \operatorname{End}_{S}(A)$ is a ring homomorphism. In subsequent sections we will further assume that $\iota$ is injective.

Definition 1. Let $(A, \iota)$ be an abelian scheme with an $R$-action, and $M$ a projective finitely presented right $R$-module. The Serre tensor construction is the abelian scheme $M \otimes_{R} A$ that represents $([4, \S 7])$ the group-functor

$$
M \otimes_{R} A: \mathrm{Sch}_{/ S} \longrightarrow \mathrm{Ab}, \quad T \mapsto M \otimes_{R} A(T) .
$$

Suppose $P$ is another ring, free of finite rank over $\mathbb{Z}$, and the right $R$-module $M$ is also a left $P$-module, such that the actions of $P$ and $R$ commute. Then $P$ acts on $M \otimes_{R} A$ via the $M$ factor. In particular if $R$ is commutative then $M \otimes_{R} A$ has an $R$-action.

Let $(A, \iota),(B, \jmath)$ be abelian schemes with $R$-actions. An $R$-linear homomorphism $\phi: A \rightarrow B$ is a homomorphism of abelian schemes satisfying $\phi \circ \iota(r)=\jmath(r) \circ \phi$ for all $r \in R$. If $f: M \rightarrow N$ is a homomorphism of projective finitely presented right $R$-modules, and $\phi: A \rightarrow B$ an $R$-linear homomorphism of abelian schemes, by $f \otimes \phi: M \otimes_{R} A \rightarrow N \otimes_{R} B$ we denote the map given on $T$-valued points by

$$
(f \otimes \phi)_{T}: M \otimes_{R} A(T) \rightarrow N \otimes_{R} B(T), \quad m \otimes a \mapsto f(m) \otimes \phi(a), \quad T \in \mathrm{Sch}_{/ S} .
$$

We often hide canonical isomorphisms, e.g. we write $A^{n}=R^{n} \otimes_{R} A$. We also write $f_{A}$ for $f \otimes \mathbb{1}_{A}$.

1.1. Homomorphisms. The key proposition is the following.

Proposition 2. Let $A$ be an abelian scheme over $S$, with action by a ring $R$, and suppose $M$ is a projective finitely presented right $R$-module. Let $B$ be another abelian scheme over $S$, with action by a ring $P$, and $N$ a projective and finitely presented right $P$-module.

(a) There is a canonical isomorphism of abelian groups

$$
\Psi: N \otimes_{P} \operatorname{Hom}_{S}(A, B) \otimes_{R} M^{\vee} \cong \operatorname{Hom}_{S}\left(M \otimes_{R} A, N \otimes_{P} B\right),
$$

mapping a pure tensor $n \otimes \phi \otimes f$ to the morphism given on $T$-valued points by

$\Psi(n \otimes \phi \otimes f)_{T}: M \otimes_{R} A(T) \rightarrow N \otimes_{P} B(T), \quad m \otimes a \mapsto n \otimes \phi(f(m) a), \quad T \in \mathrm{Sch}_{/ S}$.

(b) Suppose $P=R$ and $M, N$ are $R$-bimodules, so that $M \otimes_{R} A, N \otimes_{R} B$ acquire $R$-actions. The above isomorphism, restricted to $R$-linear homomorphisms, gives a canonical isomorphism

$$
\Psi: N \otimes_{R} \operatorname{Hom}_{R}(A, B) \otimes_{R} M^{\vee} \cong \operatorname{Hom}_{R}\left(M \otimes_{R} A, N \otimes_{R} B\right) .
$$

(c) With $M, N, R$ as in (b), suppose $R$ is moreover commutative. Then there's a canonical isomorphism of $R$-modules

$$
\operatorname{Hom}_{R}(M, N) \otimes_{R} \operatorname{Hom}_{R}(A, B) \cong \operatorname{Hom}_{R}\left(M \otimes_{R} A, N \otimes_{R} B\right),
$$

mapping $h \otimes \phi$ to the morphism given on $T$-valued points by $h \otimes \phi_{T}$, for $T \in \mathrm{Sch}_{/ S}$.

Proof. For part (a), the statement is obvious if $M$ and $N$ are free. For the general case, pick a finitely presented projective right $R$-module $M^{\prime}$, resp. $P$-module $N^{\prime}$, so that $M_{0}=M \oplus M^{\prime}$ and $N_{0}=N \oplus N^{\prime}$ are free of finite rank. Then the isomorphism

$$
N_{0} \otimes_{P} \operatorname{Hom}_{S}(A, B) \otimes_{R} M_{0}^{\vee} \cong \operatorname{Hom}_{S}\left(M_{0} \otimes_{R} A, N_{0} \otimes_{P} A\right)
$$

decomposes into a direct sum of four morphisms of abelian groups, which all must be isomorphisms, one of which coincides with the morphism in the statement. The explicit form of the map may be checked by following through the canonical isomorphisms involved.

The other parts are similar. For $(b)$, first assume $M \simeq R^{m}$ and $N \simeq R^{n}$, so that $\operatorname{Hom}\left(M \otimes_{R}\right.$ $\left.A, N \otimes_{R} B\right)$ may be identified with $M_{n}(\operatorname{Hom}(A, B))$, the additive group of $m \times n$ matrices with entries in $\operatorname{Hom}(A, B)$. The claim becomes equivalent to the fact that $R$-linear elements of $M_{n}(\operatorname{Hom}(A, B))$ 
correspond to matrices with $R$-linear entries. The general case may be deduced by picking complementary projective modules as in part (a).

For $(c)$ note that the left and right $R$-actions on $\operatorname{Hom}_{R}(A, B)$ agree by definition of $R$-linearity. The claim then follows from $(b)$ and the associativity of tensor products of $R$-bimodules, plus the fact that the canonical morphism $M^{\vee} \otimes_{R} N \rightarrow \operatorname{Hom}_{R}(M, N)$ is an isomorphism since $M$ and $N$ are finitely presented and projective.

1.2. Lie algebra and Tate module. The following lemma says taking the Tate module or Lie algebra of an abelian scheme commutes with applying Serre's construction.

Lemma 3. Let $A$ be an abelian scheme over a base $S$, equipped with an action $\iota: R \rightarrow \operatorname{End}_{S}(A)$ by a ring $R$. Suppose $M$ is a projective finitely presented right $R$-module. There is then a canonical isomorphism of group schemes

$$
T_{l}\left(M \otimes_{R} A\right) \cong M \otimes_{R} T_{l}(A),
$$

as well as a canonical isomorphism of $\mathcal{O}_{S}$-modules

$$
\operatorname{Lie}_{S}\left(M \otimes_{R} A\right) \cong M \otimes_{R} \operatorname{Lie}_{S}(A) .
$$

Proof. For any positive integer $N$, the sequence

$$
0 \rightarrow M \otimes_{R} A[N] \rightarrow M \otimes_{R} A \stackrel{N}{\rightarrow} M \otimes_{R} A \rightarrow 0
$$

is exact since $M$ is a flat $R$-module. The first claim follows by passing to the limit.

For the second assertion we use the functorial description of $\operatorname{Lie}_{S}(A)$ given by

$$
\operatorname{Lie}_{S}(A)(U)=\operatorname{ker}(A(U[\epsilon]) \rightarrow A(U)),
$$

for $U \subset S$ [8, Exp II, 3.9]. Here $A(U[\epsilon]) \rightarrow A(U)$ is induced by $U \rightarrow U[\epsilon]$, which is constructed as follows. $U[\epsilon]=U \times \operatorname{Spec}(\mathbb{Z}) \mathbb{Z}[\epsilon]$, where $\mathbb{Z}[\epsilon]$ is the ring of dual numbers. The map $U \rightarrow U[\epsilon]$ comes from applying the fibre product functor $U \times \operatorname{Spec} \mathbb{Z}-$ to the morphism $\operatorname{Spec} \mathbb{Z} \rightarrow \operatorname{Spec} \mathbb{Z}[\epsilon]$, and the latter corresponds to the ring homomorphism $\mathbb{Z}[\epsilon] \rightarrow \mathbb{Z}$ that sends $\epsilon$ to 0 . The claim follows again from the fact that $M \otimes_{R}$ - preserves kernels by flatness.

1.3. The dual abelian scheme. Recall that the dual $M^{\vee}=\operatorname{Hom}_{R}(M, R)$ of a right $R$-module $M$ is naturally a left $R$-module, with $(r \cdot f)(m)=r \cdot f(m), r \in R, f \in M^{\vee}, m \in M$.

Definition 4. A positive involution ring $(R, *)$ is a $\operatorname{ring} R$, free of finite rank over $\mathbb{Z}$, equipped with an involution $r \mapsto r^{*}$ such that $(a, b) \mapsto \operatorname{Tr}_{R_{\mathbb{Q}} / \mathbb{Q}}\left(a b^{*}\right)$ is positive-definite on $R_{\mathbb{Q}}$.

We assume $(R, *)$ is a positive involution ring. Then $M^{\vee}$ is a right $R$-module via

$$
(f \cdot r)(m)=r^{*} f(m) .
$$

By $f^{\vee}$ we denote the dual of $f: M \rightarrow N$. The map $f \mapsto f(1)^{*}$ defines a canonical isomorphism of left $R$-modules $R^{\vee} \rightarrow R$.

Let $\mathscr{P}=\mathscr{P}_{R}$ denote the category of projective finitely presented right $R$-modules. The map $M \mapsto M^{\vee}$ defines a contravariant functor from $\mathscr{P}$ to itself. We write $\left(M^{\vee}\right)^{\vee}=M$ by abuse of notation.

Fixing a base scheme $S$, by $\mathscr{A}=\mathscr{A}(S)$ we denote the category of abelian schemes and group scheme homomorphisms over $S$. The map $A \mapsto A^{\vee}$ is a contravariant functor from $\mathscr{A}$ to itself. We write $\left(A^{\vee}\right)^{\vee}=A$ by abuse.

Let $\mathscr{A}_{R}=\mathscr{A}_{R}(S)$ denote the category of pairs $(A, \iota)$, where $A \in \mathscr{A}$ is equipped with an $R$ action. The morphisms in $\mathscr{A}_{R}$ are required to be $R$-linear. Each $(A, \iota)$ has a dual $\left(A^{\vee}, \iota^{\vee}\right)$ with $\iota^{\vee}(r)=\iota\left(r^{*}\right)^{\vee}$, and $(A, \iota) \mapsto\left(A^{\vee}, \iota^{\vee}\right)$ is a contravariant functor from $\mathscr{A}_{R}$ to itself.

Let $\mathcal{S}: \mathscr{P} \times \mathscr{A}_{R} \rightarrow \mathscr{A}$ be the functor induced by Serre's construction sending $(M, A)$ to $M \otimes_{R} A$. 
Proposition 5. The following diagram commutes up to canonical isomorphism:

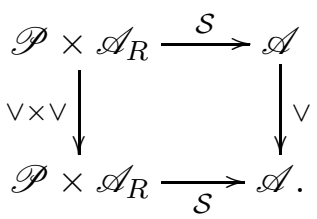

In other words, for $M \in \mathscr{P},(A, \iota) \in \mathscr{A}_{R}$, and $f \in \operatorname{Mor}(\mathscr{P}), \phi \in \operatorname{Mor}\left(\mathscr{A}_{R}\right)$, we have

$$
\left(M \otimes_{R} A\right)^{\vee} \cong M^{\vee} \otimes_{R} A^{\vee}, \quad(f \otimes \phi)^{\vee} \cong f^{\vee} \otimes \phi^{\vee} .
$$

The isomorphism $\Phi=\Phi_{M, A}: M^{\vee} \otimes_{R} A^{\vee} \rightarrow\left(M \otimes_{R} A\right)^{\vee}$ is characterized as follows. For $T \in \mathscr{A}$, and $g \otimes t \in M^{\vee} \otimes_{R} \operatorname{Hom}_{S}\left(T, A^{\vee}\right)$, the map $\Phi_{T}(g \otimes t) \in \operatorname{Hom}_{S}\left(T,\left(M \otimes_{R} A\right)^{\vee}\right)$ is the dual of the homomorphism $M \otimes_{R} A \rightarrow T^{\vee}$ given on $U$-valued points by

$$
M \otimes_{R} A(U) \rightarrow T^{\vee}(U), \quad m \otimes u \mapsto t^{\vee} \circ \iota(g(m)) \circ u, \quad U \in \mathrm{Sch}_{/ S} .
$$

Proof. Let $T$ be an abelian scheme over $S$. We have canonical isomorphisms:

$$
\begin{aligned}
\operatorname{Hom}_{S}\left(T, M^{\vee} \otimes_{R} A^{\vee}\right) & \cong M^{\vee} \otimes_{R} \operatorname{Hom}_{S}\left(T, A^{\vee}\right) & & (\text { by Proposition 2(a)) } \\
& \cong \operatorname{Hom}_{S}\left(A, T^{\vee}\right) \otimes_{R} M^{\vee} & & \left(f \otimes \phi \mapsto \phi^{\vee} \otimes f\right) \\
& \cong \operatorname{Hom}_{S}\left(M \otimes_{R} A, T^{\vee}\right) & & \text { (by Proposition 2(a)) } \\
& \cong \operatorname{Hom}_{S}\left(T,\left(M \otimes_{R} A\right)^{\vee}\right) . & & \text { (duality) }
\end{aligned}
$$

Letting $T=M^{\vee} \otimes_{R} A^{\vee}$, the canonical morphism $\Phi: M^{\vee} \otimes_{R} A^{\vee} \rightarrow\left(M \otimes_{R} A\right)^{\vee}$ corresponds to the identity element in

$$
\operatorname{Hom}_{S}\left(M^{\vee} \otimes_{R} A^{\vee}, M^{\vee} \otimes_{R} A^{\vee}\right) \cong \operatorname{Hom}_{S}\left(M^{\vee} \otimes_{R} A^{\vee},\left(M \otimes_{R} A\right)^{\vee}\right),
$$

and its inverse $\left(M \otimes_{R} A\right)^{\vee} \rightarrow M^{\vee} \otimes_{R} A^{\vee}$ corresponds to the identity in

$$
\operatorname{Hom}_{S}\left(\left(M \otimes_{R} A\right)^{\vee}, M^{\vee} \otimes_{R} A^{\vee}\right) \cong \operatorname{Hom}_{S}\left(\left(M \otimes_{R} A\right)^{\vee},\left(M \otimes_{R} A\right)^{\vee}\right),
$$

which comes from setting $T=\left(M \otimes_{R} A\right)^{\vee}$.

The explicit form of $\Phi$, as well as the relation $(f \otimes \phi)^{\vee}=f^{\vee} \otimes \phi^{\vee}$, may be checked by following through these isomorphisms carefully.

1.4. Polarizations. We recall some basic definitions and facts about polarizations of abelian schemes. Let $A$ be an abelian scheme over a base $S$. The Poincaré correspondence $\mathscr{P}_{A}$, is a universal line bundle on $A \times{ }_{S} A^{\vee}$ that induces, for any abelian scheme $B / S$, a canonical isomorphism of groups

$$
\operatorname{Hom}_{S}\left(B, A^{\vee}\right) \cong \operatorname{Corr}_{S}(A, B), \quad\left(\phi: B \rightarrow A^{\vee}\right) \mapsto\left(\mathbb{1}_{A} \times \phi\right)^{*}\left(\mathscr{P}_{A}\right) .
$$

Here $\operatorname{Corr}_{S}(A, B)$ denotes the group of correspondences on $A \times_{S} B$ [6, I.1.7].

Let $\Delta: A \rightarrow A \times{ }_{S} A$ be the diagonal. For a morphism $f: A \rightarrow A^{\vee}$ of abelian schemes, let $\mathcal{L}_{f}$ denote the correspondence $(1 \times f)^{*} \mathscr{P}_{A}$ on $A \times_{S} A$, and $\mathscr{L}_{f}=\Delta^{*}\left(\mathcal{L}_{f}\right)$ the associated line bundle on $A$. If $g: B \rightarrow A$ is a homomorphism of abelian schemes, then the pullbacks of $\mathscr{L}_{f}$ and $\mathcal{L}_{f}$ under $g$ and $g \times g$ correspond to the map $g^{\vee} \circ f \circ g: B \rightarrow B^{\vee}$. In other words:

$$
g^{*} \mathscr{L}_{f}=\mathscr{L}_{g \vee \circ f \circ g}, \quad(g \times g)^{*} \mathcal{L}_{f}=\mathcal{L}_{g \vee \circ f \circ g} .
$$

If $\mathcal{L}$ is a correspondence on $A \times_{S} A$, its associated map $\lambda: A \rightarrow A^{\vee}$ is symmetric if and only if $\mathcal{L}$ is a symmetric correspondence, meaning $s^{*}(\mathcal{L}) \simeq \mathcal{L}$ with $s: A \times A \rightarrow A \times A$ the coordinate flip map.

A polarization on an abelian variety $A_{0}$ is a symmetric homomorphism $\lambda: A_{0} \rightarrow A_{0}^{\vee}$ associated to a correspondence $\mathcal{L}_{\lambda}$ as above, such that the line bundle $\mathscr{L}_{\lambda}=\Delta^{*}\left(\mathcal{L}_{\lambda}\right)$ on $A_{0}$ is ample. A polarization on the abelian scheme $A$ is a symmetric homomorphism $\lambda: A \rightarrow A^{\vee}$ such that for 
every geometric point $\bar{s} \rightarrow S, \lambda_{\bar{s}}: A_{\bar{s}} \rightarrow A_{\bar{s}} \vee$ is a polarization of abelian varieties. A principal polarization is one that is an isomorphism.

The choice of a polarization on an abelian scheme $A$ induces a Rosati involution $\phi \mapsto \rho(\phi)$ on $\operatorname{End}^{0}(A)=\operatorname{End}(A) \otimes \mathbb{Q}$, determined by the commutativity of the following diagram in the isogeny category:

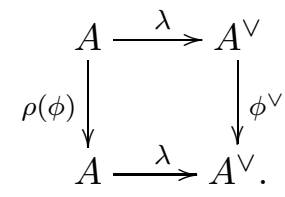

Let $(R, *)$ be a ring with involution, and $(A, \iota)$ an abelian scheme with $R$-action. Then the dual $A^{\vee}$ has an $R$-action $\iota^{\vee}$ given by $\iota^{\vee}(r)=\iota\left(r^{*}\right)^{\vee}$. A polarization $\lambda: A \rightarrow A^{\vee}$ is $R$-linear if and only if $\lambda \circ \iota(r)=\iota\left(r^{*}\right)^{\vee} \circ \lambda$ for all $r \in R$. By the above diagram, this is equivalent to $\iota\left(r^{*}\right)=\rho(\iota(r))$, so that $\lambda$ is $R$-linear if and only if $\iota$ maps $*$ to the Rosati $\rho$.

Let $(A, \iota, \lambda)$ be as above, with $\lambda$ an $R$-linear polarization, and denote by $\mathcal{L}=\mathcal{L}_{\lambda}$ the correspondence associated to it. The behaviour of the pullback of $\mathcal{L}$ under the product $f \times g$ of various maps $f, g \in \operatorname{End}(A)$ is described as follows.

Proposition 6. The map $l: \operatorname{End}(A) \times \operatorname{End}(A) \rightarrow \operatorname{Corr}(A, A)$ given by $l(x, y)=(x \times y)^{*} \mathcal{L}$ satisfies the linearity relations

$$
\begin{aligned}
& l(x+y, z)=l(x, z) \otimes l(y, z) \\
& l(x, y+z)=l(x, y) \otimes l(x, z) \\
& l(x, y \circ \iota(r))=l\left(x \circ \iota\left(r^{*}\right), y\right),
\end{aligned}
$$

for all $x, y, z \in \operatorname{End}(A)$, and $r \in R$.

Proof. A corollary of the theorem of the cube [21, p.59] states that if $f, g, h: X \rightarrow Y$ are maps of abelian varieties, and $\mathscr{L}$ is a line bundle on $B$, then:

$$
(f+g+h)^{*} \mathscr{L} \cong(f+g)^{*} \mathscr{L} \otimes(g+h)^{*} \mathscr{L} \otimes(f+h)^{*} \mathscr{L} \otimes f^{*} \mathscr{L}^{-1} \otimes g^{*} \mathscr{L}^{-1} \otimes h^{*} \mathscr{L}^{-1}
$$

The first property follows from the above applied to $f=(x \times 0), g=(y \times 0), h=(0 \times z)$, and $\mathscr{L}=\mathcal{L}_{\lambda}$, with the second being similar. The third property follows from the $R$-linearity of $\lambda$.

Let $(R, *)$ be a ring with an involution, and $M$ a projective finitely presented right $R$-module. A $\mathbb{Z}$-bilinear form $F: M \times M \rightarrow R$ is called sesquilinear if $F\left(m r, n r^{\prime}\right)=r^{*} F(m, n) r^{\prime}$ for all $m, n \in M$, and $r, r^{\prime} \in R$. By the tensor-hom adjunction formula, sesquilinear forms $F$ are in bijection with linear maps $f \in \operatorname{Hom}_{R}\left(M, M^{\vee}\right)$ via $f(m)(n)=F(m, n)$. A sesquilinear form $F$ is called hermitian if $F(m, n)^{*}=F(n, m)$ for all $m, n \in M$.

Let $p: R^{n} \rightarrow M$ be a fixed presentation of $M$, and $e_{1}, \cdots, e_{n}$ the image of the standard basis elements. A sesquilinear form $F$ on $M$ is hermitian if and only if the $n \times n$ matrix with entries $F\left(e_{i}, e_{j}\right)$ is hermitian. That is, if and only if $F\left(e_{i}, e_{j}\right)=F\left(e_{j}, e_{i}\right)^{*}$ for $1 \leq i, j \leq n$.

If $p_{A}=p \otimes \mathbb{1}_{A}: A^{n} \rightarrow M \otimes_{R} A^{n}$, then $\psi=p_{A}^{\vee}(f \otimes \lambda) \circ p_{A} \in \operatorname{End}_{S}\left(A^{n},\left(A^{\vee}\right)^{n}\right)$. The map $\psi$ is determined by an $n \times n$ matrix of morphisms $\psi_{i j}: A \rightarrow A^{\vee}$.

Proposition 7. With $f \otimes \lambda$ and $\psi$ as above, the following are equivalent:

(1) $f \otimes \lambda$ is symmetric.

(2) $\psi$ is symmetric.

(3) $\psi_{i j}=\left(\psi_{j i}\right)^{\vee}$ for $1 \leq i, j \leq n$.

(4) $f$ is hermitian.

Proof. This is left to the reader as an exercise in linear algebra. 
Now we recall Jordan algebras and the notion of positivity in a formally real Jordan algebra over $\mathbb{R}$. We relate positivity in certain matrix Jordan algebras with the usual notion of a positive-definite matrix. Then we define positive-definite $R$-hermitian structures $h: M \rightarrow M^{\vee}$, which correspond to polarizations on $M \otimes_{R} A$. The reference for this material is [1] and [18].

Definition 8. Let $k$ be a field, with $\operatorname{char}(k) \neq 2$, and let $R$ be an algebra over $k$, not necessarily associative, with multiplication denoted by $\circ$. Then $(R, \circ)$ is called a Jordan algebra if it is commutative, and

$$
(u \circ u) \circ(u \circ v)=u \circ((u \circ u) \circ v), \quad \forall u, v \in R .
$$

A Jordan algebra $R$ is called formally real if for all $u, v \in R$,

$$
u \circ u+v \circ v=0 \Longleftrightarrow u=v=0 .
$$

Any associative $k$-algebra $R$ can be turned into a Jordan algebra $(R, \circ)$ by setting

$$
x \circ y=\frac{1}{2}(x y+y x) .
$$

We restrict to the case where $k$ is subfield of $\mathbb{R}$, so that it makes sense to speak of positive elements of $k$. Let $R$ be a finite associative $k$-algebra equipped with a positive involution $r \mapsto r^{*}$, so that $(x, y) \mapsto \operatorname{Tr}_{R / k}\left(y^{*} x\right)$ is positive definite. The elements of $R$ fixed by the involution are called symmetric. The positivity of the involution implies that the subalgebra of symmetric elements $S \subset R$ is formally real. For any $n>0$, the matrix algebra $M_{n}(R)$ inherits a positive involution $X \mapsto X^{*}$ from $R$, given by $\left(X_{i j}\right) \mapsto\left(X_{j i}^{*}\right)$. Its symmetric elements are the formally real Jordan algebra $H_{n}(R)$ of $n \times n$ hermitian matrices.

Definition 9. An element $u$ of a formally real Jordan algebra over the real numbers $\mathbb{R}$ is called positive, and denoted $u>0$, if all the eigenvalues of the $\mathbb{R}$-linear map $L_{u}(v)=u \circ v$ are positive.

If $R$ is any one of: the real numbers $\mathbb{R}$, complex numbers $\mathbb{C}$, or the standard quaternions $\mathbb{H}$, it can be considered as a Jordan algebra over $\mathbb{R}$, with a positive involution given by the identity map on $\mathbb{R}$, complex conjugation on $\mathbb{C}$, and the standard involution on $\mathbb{H}$. In all three cases, the matrices in $H_{n}(R)$ are unitarily diagonalizable. For a matrix $X \in H_{n}(R)$, the eigenvalues of the operator $L_{X}: H_{n}(R) \rightarrow H_{n}(R), L_{X}(Y)=X \circ Y=\frac{1}{2}(X Y+Y X)$ are $\frac{1}{2}\left(d_{i}+d_{j}\right)$ where $d_{i}$ are the ordinary eigenvalues of $X$ as a matrix. It follows that $X>0$ in $H_{n}(R)$ if and only if $X$ is a positive definite matrix in the usual sense.

Now suppose $R=K$ is a CM field, with maximal totally real subfield $F$. Then $K$ is an algebra over $F$, with complex conjugation defining a positive involution. The formally real Jordan algebra $H_{n}(K) \otimes \mathbb{R}$ over $\mathbb{R}$ is isomorphic to a product of algebras $H_{n}(\mathbb{R})$ and $H_{n}(\mathbb{C})$, one for each embedding of $F$ in $\mathbb{R}$. A matrix $X \in H_{n}(K)$ is positive in $H_{n}(K) \otimes \mathbb{R}$ if and only if it is positive in each factor of the product. It follows that $X>0$ if and only if the eigenvalues of $X$ are totally positive algebraic numbers.

If $A$ is an abelian variety with a polarization, $\operatorname{End}(A)_{\mathbb{Q}}$ is equipped with a Rosati involution. By a symmetric element of $\operatorname{End}(A)$, resp. $\operatorname{End}(A)_{\mathbb{Q}}$, we mean one that is fixed by the Rosati involution. We denote such elements by $\operatorname{End}(A)^{\mathrm{sym}}, \operatorname{resp} . \operatorname{End}(A)_{\mathbb{Q}}^{\mathrm{sym}}$.

We recall a result characterizing ample line bundles on abelian varieties. The following theorem is quoted from [21, p.208], where the term totally positive is used for what we call positive.

Theorem 10 (Ampleness Criterion). For $r \in \operatorname{End}(A)^{\mathrm{sym}}$, the line bundle $\mathscr{L}_{\lambda \circ \mathrm{r}} \in \operatorname{Pic}(A)$ is ample if and only if $r$ is positive in the formally real Jordan algebra $\operatorname{End}(A) \otimes \mathbb{R}$.

Corollary 11. Let $S$ be a connected scheme, $A$ an abelian scheme over $S, \lambda: A \rightarrow A^{\vee}$ a polarization, and $r \in \operatorname{End}_{S}(A)$. Then $\lambda \circ r$ is a polarization if and only if $r$ is symmetric and positive in the formally real Jordan algebra $\operatorname{End}_{S}(A) \otimes \mathbb{R}$. 
Proof. Using the rigidity lemma of [20, Ch. 6], one easily reduces to the case where $S$ is the spectrum of an algebraically closed field. Let $r \mapsto r^{*}$ denote the Rosati involution of $\lambda$. For $r \in \operatorname{End}_{S}(A)$, $\lambda \circ r$ is symmetric if and only if $r$ is symmetric: this follows from $(\lambda \circ r)^{\vee}=r^{\vee} \circ \lambda=\lambda \circ r^{*}$, and the fact that $\lambda$ is an isogeny. Now suppose $r$ is symmetric. By Mumford's ampleness criterion, the line bundle $\mathscr{L}_{\lambda \circ r}$ corresponding to $\lambda \circ r$ is ample if and only if $r$ is positive in $\operatorname{End}(A) \otimes \mathbb{R}$. Hence $r$ is symmetric and positive in $\operatorname{End}_{S}(A) \otimes \mathbb{R}$, if and only if $\lambda \circ r$ is symmetric and $\mathscr{L}_{\lambda \circ r}$ is ample.

Definition 12. A positive involution $\operatorname{ring}(R, *)$ is said to satisfy property $(\mathbf{P})$, if for every matrix $Q$ in $G L_{n}\left(R_{\mathbb{Q}}\right)$ the hermitian matrix $Q^{*} Q \in H_{n}\left(R_{\mathbb{Q}}\right)$ is positive in the formally real Jordan algebra $H_{n}(R) \otimes \mathbb{R}$.

The following lemma shows that property $(\mathrm{P})$ holds in all cases of interest.

Lemma 13. Let $(R, *)$ be a positive involution ring. Suppose an abelian scheme $A / S$ exists that admits an $R$-action, with an $R$-linear polarization $\lambda$. Then $R$ necessarily satisfies property $(P)$.

Proof. Since for any point $s$ of the base scheme $S$ the map $\operatorname{End}_{S}(A) \rightarrow \operatorname{End}_{k(s)}\left(A_{s}\right)$ is injective, we can assume $A$ is an abelian variety. Let $Q \in G L_{n}\left(R_{\mathbb{Q}}\right)$. After multiplying by a positive integer we can assume $Q$ has entries in $R$, and so defines an $R$-linear isogeny $\phi: A^{n} \rightarrow A^{n}$. Since the $R$-linear map $\lambda^{n}: A^{n} \rightarrow\left(A^{\vee}\right)^{n}$ is a polarization on $A^{n}$, the line bundle $\mathscr{L}_{\lambda^{n}}$ is ample, therefore the line bundle $\mathscr{L}_{\phi^{\vee} \circ \lambda^{n} \circ \phi}=\phi^{*} \mathscr{L}_{\lambda^{n}}$ is also ample, as $\phi$ is an isogeny. Since $\lambda$ is $R$-linear, $\phi^{\vee} \circ \lambda^{n} \circ \phi=\lambda^{n} \circ \psi$, where $\psi: A^{n} \rightarrow A^{n}$ is given by the hermitian matrix $Q^{*} Q$ with coefficients in $R$. Then by the ampleness criterion, the symmetric element $\psi$ is positive in the formally real Jordan algebra $H_{n}\left(R_{\mathbb{Q}}\right) \otimes \mathbb{R} \subset \operatorname{End}\left(A^{n}\right) \otimes \mathbb{R}$, where it is identified with $Q^{*} Q$.

A projective finitely presented module $M$ over a Dedekind domain is automatically a lattice in $M_{\mathbb{Q}}$. For general rings $R$, we make the following definition.

Definition 14. A lattice $M$ over $R$ is a projective finitely presented right $R$-module $M$ such that $M_{\mathbb{Q}}$ is free over $R_{\mathbb{Q}}$.

The key positivity notion is as follows.

Definition 15. Suppose $(R, *)$ satisfies property $(\mathrm{P})$. A hermitian lattice $(M, h)$ is positive definite if for some isomorphism $\eta: R_{\mathbb{Q}}^{n} \rightarrow M_{\mathbb{Q}}$, the standard matrix $T \in H_{n}\left(R_{\mathbb{Q}}\right)$ of the map $\eta^{\vee} \circ h_{\mathbb{Q}} \circ \eta$ is positive in the formally real Jordan algebra $H_{n}(R) \otimes \mathbb{R}$.

The following lemma shows that the above definition does not depend on the choice of $\eta$.

Lemma 16. Let $(R, *)$ be a positive involution ring with property $(P)$. Let $Q \in G L_{n}\left(R_{\mathbb{Q}}\right)$ and $T \in H_{n}\left(R_{\mathbb{Q}}\right)$. Then $T>0$ in $H_{n}\left(R_{\mathbb{Q}}\right) \otimes \mathbb{R}$ if and only if $Q T Q^{*}>0$.

Proof. Let $J_{1}$ denote the Jordan algebra $H_{n}\left(R_{\mathbb{Q}}\right)$, and $J_{Q Q^{*}}$ the algebra with the same underlying abelian group as $J_{1}$ and product defined by

$$
X \circ_{Q Q^{*}} Y=\frac{1}{2}\left(X\left(Q Q^{*}\right)^{-1} Y+Y\left(Q Q^{*}\right)^{-1} X\right) .
$$

Then $J_{Q Q^{*}}$ is a formally real Jordan algebra with unit element $Q Q^{*}$, and the map $T \mapsto Q T Q^{*}$ is an isotopy of Jordan algebras $J_{1} \rightarrow J_{Q Q^{*}}[18$, p.14]. It can be extended $\mathbb{R}$-linearly to an isotopy $J_{1} \otimes \mathbb{R} \rightarrow J_{Q Q^{*}} \otimes \mathbb{R}$ of formally real Jordan algebras over $\mathbb{R}$.

The set of positive elements of a formally real Jordan algebra $J$ over $\mathbb{R}$ is an open convex cone, identified with the connected component of the identity in the units $J^{\times}$of $J$ [18, p.18]. An isotopy of Jordan algebras preserves the positive cone.

The positive cone of $J_{Q Q^{*}} \otimes \mathbb{R}$, which is the same topological space as $J_{1} \otimes \mathbb{R}$, is the connected component of its identity element $Q Q^{*}$. Since $(R, *)$ satisfies property $(\mathrm{P}), Q Q^{*}$ is positive in $J_{1} \otimes \mathbb{R}$, therefore it lies in the same connected component as the identity element of $J_{1} \otimes \mathbb{R}$. Thus 
the isotopy $T \mapsto Q T Q^{*}$ maps the positive cone of $J_{1} \otimes \mathbb{R}$ to itself, and so $T>0$ if and only if $Q T Q^{*}>0$.

The main result of this section (Theorem A from the introduction), is as follows.

Theorem 17. Let $(R, *)$ be a positive involution ring, $(A, \iota)$ an abelian scheme with an $R$-action, and $M$ a lattice over $R$. Suppose $\lambda: A \rightarrow A^{\vee}$ is an R-linear polarization and $h: M \rightarrow M^{\vee}$ is an R-linear map. Then $h \otimes \lambda: M \otimes_{R} A \rightarrow M^{\vee} \otimes_{R} A^{\vee}$ is a polarization if and only if $(M, h)$ is hermitian and positive definite.

Proof. By Proposition 7, $h \otimes \lambda$ is symmetric if and only if $h$ is hermitian, so we can assume this is the case. Such a symmetric map is by definition a polarization if and only if it is a polarization of abelian varieties on geometric fibres. Since Serre's construction commutes with fibre products, $h \otimes \lambda$ is a polarization if and only if $(h \otimes \lambda)_{s} \cong h \otimes \lambda_{s}$ is a polarization for all geometric points $s \rightarrow S$. Thus we can assume $A$ is an abelian variety over an algebraically closed field.

We fix an isomorphism $\eta: R_{\mathbb{Q}}^{n} \rightarrow M_{\mathbb{Q}}$, through which we identify $M \subset M_{\mathbb{Q}}$ with its pre-image in $R_{\mathbb{Q}}^{n}$. Let $T \in H_{n}\left(R_{\mathbb{Q}}\right)$ be the matrix of $\eta^{\vee} \circ h_{\mathbb{Q}} \circ \eta$ as in Definition 15. Since the inclusion $M \subset R_{\mathbb{Q}}^{n}$ becomes an isomorphism after tensoring with $\mathbb{Q}$, there is a positive integer $k$ such that $k R^{n} \subset M$. Let $\kappa: R^{n} \rightarrow M$ denote multiplication by $k$. Using the identification $A^{n}=R^{n} \otimes_{R} A$, we obtain an isogeny $\kappa_{A}=\kappa \otimes \mathbb{1}_{A}: A^{n} \rightarrow M \otimes_{R} A$. Now let $f=\kappa^{\vee} \circ h \circ \kappa$ and consider the map $f \otimes \lambda: A^{n} \rightarrow\left(A^{\vee}\right)^{n}$. Since $h \otimes \lambda$ is symmetric, so is $f \otimes \lambda=\kappa_{A}^{\vee} \circ(h \otimes \lambda) \circ \kappa_{A}$. The matrix of $f$ is a positive integer multiple of $T$, so $\left(R^{n}, f\right)$ is positive-definite if and only if $(M, h)$ is. Since $\kappa_{A}$ is an isogeny, the line bundle $\kappa_{A}^{*}\left(\mathscr{L}_{h \otimes \lambda}\right)=\mathscr{L}_{f \otimes \lambda}$ is ample if and only if $\mathscr{L}_{h \otimes \lambda}$ is ample. It follows that it's enough to prove the theorem for $\left(R^{n}, f\right)$ in place of $(M, h)$.

Now $f \otimes \lambda: A^{n} \rightarrow\left(A^{\vee}\right)^{n}$ factors as $\lambda^{n} \circ f_{A}$, where $\lambda^{n}: A^{n} \rightarrow\left(A^{\vee}\right)^{n}$ is the product polarization of $A^{n}$ obtained from $\lambda$. By Corollary 11, $\lambda^{n} \circ f_{A}$ is a polarization if and only if $f_{A} \in \operatorname{End}\left(A^{n}\right)$ is positive in the formally real Jordan algebra $\operatorname{End}\left(A^{n}\right) \otimes \mathbb{R}$. It remains to show this is the case if and only if $f$ is positive definite.

Let us use $*$ again to denote the Rosati involution on $\operatorname{End}(A) \otimes \mathbb{Q}$ induced by $\lambda$. The algebra isomorphism $\left.M_{n}(\operatorname{End}(A) \otimes \mathbb{Q})\right) \cong \operatorname{End}\left(A^{n}\right) \otimes \mathbb{Q}$ identifies the Rosati involution induced by $\lambda^{n}$ on $\operatorname{End}\left(A^{n}\right) \otimes \mathbb{Q}$ with the positive involution $\left(X_{i j}\right) \rightarrow\left(X_{j i}^{*}\right)$ on $M_{n}(\operatorname{End}(A) \otimes \mathbb{Q})$. Thus the symmetric elements in $\operatorname{End}\left(A^{n}\right) \otimes \mathbb{Q}$ are identified with hermitian matrices $H_{n}(\operatorname{End}(A) \otimes \mathbb{Q})$. The map $f \mapsto f_{A}$ is a morphism of formally real Jordan algebras $H_{n}\left(R_{\mathbb{Q}}\right) \rightarrow H_{n}(\operatorname{End}(A) \otimes \mathbb{Q})$. It is the same as the map induced by the $R$-action $\iota: R \hookrightarrow \operatorname{End}(A)$, so it is in particular injective. Now considering $H_{n}\left(R_{\mathbb{Q}}\right)$ as a subalgebra of $\operatorname{End}\left(A^{n}\right) \otimes \mathbb{Q}$, it follows that $f_{A}>0$ in $\operatorname{End}\left(A^{n}\right) \otimes \mathbb{R}$ if and only if $f>0$ in $H_{n}(R) \otimes \mathbb{R}$. In other words, $f \otimes \lambda$ is a polarization if and only if $f$ is positive-definite, and so the same holds for $h$.

Under some extra assumptions, we can extend this theorem further to characterize principal polarizations. Recall that a hermitian form $h: M \rightarrow M^{\vee}$ is called non-degenerate if it's an isomorphism.

Proposition 18. Under the conditions of Theorem [17, suppose that furthermore $\lambda: A \rightarrow A^{\vee}$ is principal, and that either:

(i) The (left) $R$-module of endomorphisms $\operatorname{End}_{S}(A)$ is faithfully flat, or

(ii) $R$ is commutative, and the $R$-module of $R$-linear endomorphisms $\operatorname{End}_{R}(A)$ is faithfully flat.

Then $h \otimes \lambda$ is a principal polarization if and only if $h$ is a non-degenerate positive definite hermitian form.

Proof. It's clear that if $h$ is non-degenerate and $\lambda$ is principal, $h \otimes \lambda$ is an isomorphism. Conversely, suppose $h \otimes \lambda$ is an isomorphism. Since $h \otimes \lambda$ factors as a composition of the isomorphism $\mathbb{1}_{M} \otimes \lambda$ : 
$M \otimes_{R} A \rightarrow M \otimes_{R} A^{\vee}$ with the map $h \otimes_{A}: M \otimes_{R} A \rightarrow M^{\vee} \otimes_{R} A$, the latter is also an isomorphism. Now consider this isomorphism on the $A$-valued points of $M \otimes_{R} A$ :

$$
\left(h \otimes \mathbb{1}_{A}\right)_{A}: M \otimes_{R} \operatorname{End}_{S}(A) \stackrel{\sim}{\longrightarrow} M^{\vee} \otimes_{R} \operatorname{End}_{S}(A), \quad m \otimes \phi \mapsto h(m) \otimes \phi .
$$

Evidently, this is the tensor product of the map of right $R$-modules $h: M \rightarrow M^{\vee}$ with the left $R$-module $\operatorname{End}_{S}(A)$. If the latter is faithfully flat, $h$ is an isomorphism.

Now suppose $R$ is commutative. Then $M$ is a bimodule, $M \otimes_{R} A$ and $M^{\vee} \otimes_{R} A^{\vee}$ inherit $R$ actions, and the isomorphisms $h \otimes \lambda, h \otimes \mathbb{1}_{A}$ and $\mathbb{1}_{M} \otimes \lambda$ are all $R$-linear. Consider the commutative diagram

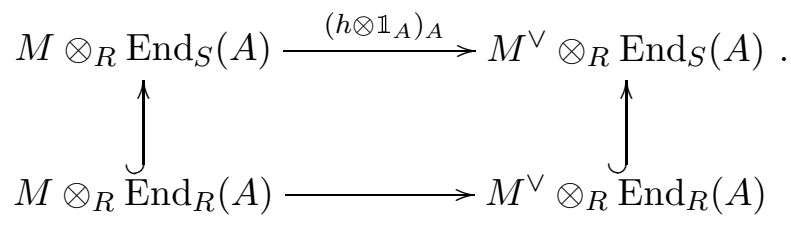

Knowing the top arrow is an isomorphism, we claim the bottom one is also one. Injectivity is clear from the diagram, so we must show surjectivity.

Let $\Psi: M^{\vee} \otimes_{R} A \rightarrow M \otimes_{R} A$ be the inverse of $h \otimes \mathbb{1}_{A}$. Since $h \otimes \mathbb{1}_{A}$ is $R$-linear, so is $\Psi$. By Proposition 2(c) it corresponds to an element of $\operatorname{Hom}_{R}\left(M^{\vee}, M\right) \otimes_{R} \operatorname{End}_{R}(A)$, which is of the form $\sum_{i} \alpha_{i} \otimes s_{i}$, for $R$-linear $\alpha_{i}: M^{\vee} \rightarrow M$ and $s_{i} \in \operatorname{End}_{R}(A)$. Then on $T$-valued points of $M^{\vee} \otimes_{R} A$, $\Psi$ is given by:

$$
\Psi_{T}: M^{\vee} \otimes_{R} A(T) \rightarrow M \otimes_{R} A(T), \quad f \otimes t \mapsto \sum_{i} \alpha_{i}(f) \otimes\left(s_{i} \circ t\right) .
$$

Letting $T=A$, we consider the map $\Psi_{A}$ restricted to $M^{\vee} \otimes_{R} \operatorname{End}_{R}(A) \subset M^{\vee} \otimes_{R} \operatorname{End}(A)$. If $t \in$ $\operatorname{End}_{R}(A)$, we also have $s_{i} \circ t \in \operatorname{End}_{R}(A)$, and so $\Psi(f \otimes t)=\sum_{i} \alpha_{i}(f) \otimes\left(s_{i} \circ t\right) \in M \otimes_{R} \operatorname{End}_{R}(A)$. This shows the inverse of $\left(h \otimes \mathbb{1}_{A}\right)_{A}$, when restricted to $M^{\vee} \otimes_{R} \operatorname{End}_{R}(A)$, takes values in $M \otimes_{R} \operatorname{End}_{R}(A)$. In other words $\left(h \otimes \mathbb{1}_{A}\right)_{A}$ restricted to $M \otimes_{R} \operatorname{End}_{R}(A)$ is surjective onto $M^{\vee} \otimes_{R} \operatorname{End}_{R}(A)$. This proves the map

$$
\left(h \otimes \mathbb{1}_{A}\right)_{A}: M \otimes_{R} \operatorname{End}_{R}(A) \longrightarrow M^{\vee} \otimes_{R} \operatorname{End}_{R}(A), \quad m \otimes s \mapsto h(m) \otimes s
$$

is an isomorphism. Now, the above map is just the tensor product of $h$ with the identity map of the $R$-module $\operatorname{End}_{R}(A)$. Thus if the latter is faithfully flat, $h$ is an isomorphism.

We note that in particular the proposition applies when $A$ is an abelian scheme with CM by the ring of integers $R=\mathcal{O}_{K}$ of a CM field $K$, since then $\operatorname{End}_{R}(A)=R$.

\section{Tensor Product of Categories}

In this section we define the action of a monoidal category on another category, and the tensor product of two categories with such actions. Then we assume the monoidal category is a 2-group, which is to say its objects are invertible with respect to the monoidal product, and we show the morphisms of the tensor product in this case have a concise form. With the application to moduli spaces in mind, we assume one of the tensor factors is a groupoid and the other is fibred in groupoids over some base category. Then we show under certain conditions the resulting tensor product is also fibred in groupoids over the same base.

2.1. Definitions. A monoidal category is a category equipped with a product on objects resembling the tensor product of modules. We recall the definition from [17, p. 162]. 
Definition 19. A monoidal category is a category $\mathcal{C}$ equipped with the following data: a bifunctor $\square: \mathcal{C} \times \mathcal{C} \rightarrow \mathcal{C}$, an identity object $e \in \mathcal{C}$, and for all $a, b, c \in \mathcal{C}$ a canonical associator isomorphism

along with left and right unitor isomorphisms

$$
\alpha_{a, b, c}:(a \square b) \square c \stackrel{\sim}{\longrightarrow} a \square(b \square c),
$$

$$
\lambda_{a}: e \square a \stackrel{\sim}{\longrightarrow} a, \quad \rho_{a}: a \square e \stackrel{\sim}{\longrightarrow} a .
$$

The isomorphisms are required to satisfy the following pentagon and triangle relations:
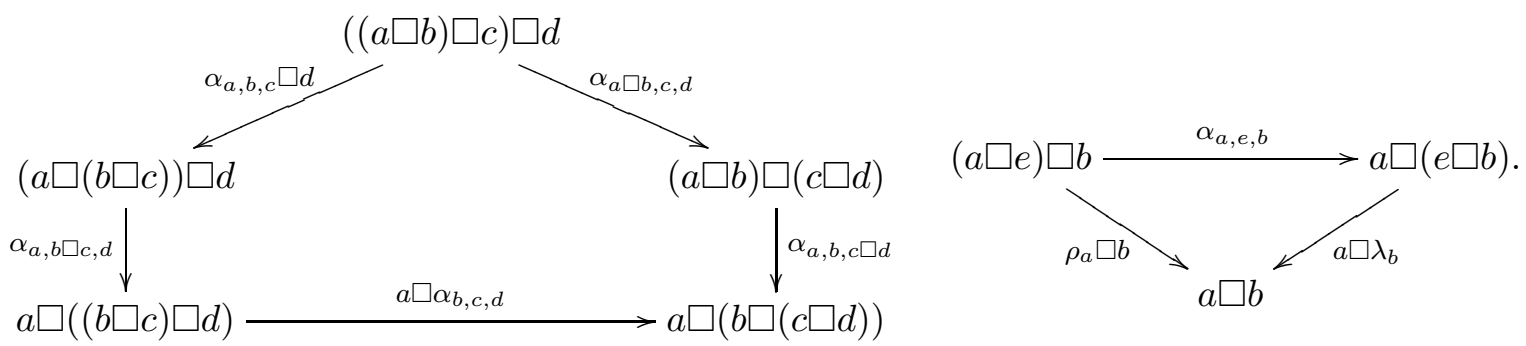

We will omit the symbol $\square$ and write $a b$ instead of $a \square b$ for short.

Definition 20. A left action of a monoidal category $\mathcal{C}$ on a category $\mathcal{X}$ is the data consisting of: a bifunctor $\mathcal{C} \times \mathcal{X} \rightarrow \mathcal{X}:(a, X) \mapsto a X$, and for all $a, b \in C, X \in \mathcal{X}$, canonical associator and left unitor isomorphisms

$$
\alpha_{a, b, X}:(a b) X \stackrel{\sim}{\longrightarrow} a(b X), \quad \lambda_{X}: e X \stackrel{\sim}{\longrightarrow} X
$$

satisfying the pentagon and triangle relations

$$
\alpha_{a, b, c X} \circ \alpha_{a b, c, X}=a \alpha_{b, c, X} \circ \alpha_{a, b c, X} \circ \alpha_{a, b, c} X, \quad a \lambda_{X} \circ \alpha_{a, e, X}=\rho_{a} X .
$$

A right action of a monoidal category $\mathcal{C}$ on a category $\mathcal{Y}$ is defined similarly, as a bifunctor $\mathcal{Y} \times \mathcal{C} \rightarrow \mathcal{Y}:(Y, a) \mapsto Y a$, with canonical associator isomorphisms $\alpha_{Y, a, b}:(Y a) b \stackrel{\sim}{\longrightarrow} Y(a b)$, and right unitors $\rho_{Y}: Y e \stackrel{\sim}{\longrightarrow} Y$, satisfying the analogous pentagon and triangle relations.

By the coherence theorem of Mac Lane [17, p.165], the pentagon and triangle relations are enough to ensure that all other expected associativity relations hold up to canonical isomorphisms.

We want to a define a tensor product of categories over a monoidal category. Such tensor products are usually defined for additive categories [24, 5]. For example, for $k$-linear categories where $k$ is a field, they have explicit constructions via generators and relations [27. We require a similar notion, but for categories with no enriched structure. For this purpose we imitate the explicit construction in [27, but leave out the additive features.

Definition 21. Let $\mathcal{C}$ be a monoidal category, and let $\mathcal{X}$ (resp. $\mathcal{Y}$ ) be a category with a right (resp. left) action of $\mathcal{C}$. The tensor product $\mathcal{X} \otimes_{\mathcal{C}} \mathcal{Y}$ is defined as the following category. The objects consist of symbols $X \otimes Y$, for $X \in \mathrm{Ob}(\mathcal{X})$ and $Y \in \mathrm{Ob}(\mathcal{Y})$. The morphisms are words, modulo relations, in the following families of generator symbols:

I. Symbols of the form

$$
\phi \otimes \psi: X \otimes Y \rightarrow X^{\prime} \otimes Y^{\prime}
$$

for all $\left(\phi: X \rightarrow X^{\prime}\right) \in \operatorname{Mor}(\mathcal{X}),\left(\psi: Y \rightarrow Y^{\prime}\right) \in \operatorname{Mor}(\mathcal{Y})$.

II. Associators symbols

$$
\alpha_{X, a, Y}: X a \otimes Y \rightarrow X \otimes a Y
$$

and their inverses

$$
\alpha_{X, a, Y}^{\prime}: X \otimes a Y \rightarrow X a \otimes Y
$$

for all $X \in \mathcal{X}, Y \in \mathcal{Y}, a \in \mathcal{C}$.

The relations imposed are: 
I. Functoriality of $\otimes$ :

$\mathbb{1}_{X} \otimes \mathbb{1}_{Y}=\mathbb{1}_{X \otimes Y}, \quad(\phi \otimes \psi) \circ\left(\phi^{\prime} \otimes \psi^{\prime}\right)=\left(\phi \circ \phi^{\prime}\right) \otimes\left(\psi \circ \psi^{\prime}\right), \quad \phi \in \operatorname{Mor}(\mathcal{X}), \quad \psi \in \operatorname{Mor}(\mathcal{Y})$.

II. Naturality of associators: commutativity of the diagram

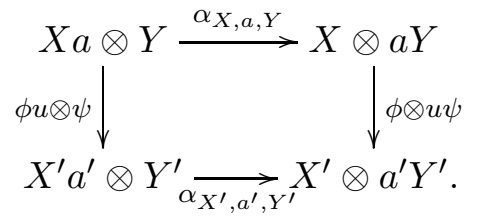

for all $\left(\phi: X \rightarrow X^{\prime}\right) \in \operatorname{Mor}(\mathcal{X}),\left(\psi: Y \rightarrow Y^{\prime}\right) \in \operatorname{Mor}(\mathcal{Y}),\left(u: a \rightarrow a^{\prime}\right) \in \operatorname{Mor}(\mathcal{C})$.

III. Isomorphic property of associators:

$\alpha_{X, a, Y} \circ \alpha_{X, a, Y}^{\prime}=\mathbb{1}_{X \otimes a Y}, \quad \alpha_{X, a, Y}^{\prime} \circ \alpha_{X, a, Y}=\mathbb{1}_{X a \otimes Y}, \quad$ for all $X \in \mathcal{X}, Y \in \mathcal{Y}, a \in \mathcal{C}$.

IV. The pentagon and triangle relations:

$\alpha_{X, a, b Y} \circ \alpha_{X a, b, Y}=\left(\mathbb{1}_{X} \otimes \alpha_{a, b, Y}\right) \circ \alpha_{X, a b, Y} \circ\left(\alpha_{X, a, b} \otimes \mathbb{1}_{Y}\right), \quad\left(\mathbb{1}_{X} \otimes \lambda_{Y}\right) \circ \alpha_{X, e, Y}=\rho_{X} \otimes \mathbb{1}_{Y}$.

Again by Mac Lane's coherence theorem [17, p. 165] the pentagon and triangle relations above, together with their counterparts in the definitions of the monoidal category $C$, and the actions of $C$ on $\mathcal{X}$ and $\mathcal{Y}$, imply that all expected associativity relations hold up to canonical isomorphism. Thus for instance, up to canonical isomorphism, the object $X a_{1} a_{2} . . a_{r} \otimes b_{1} b_{2} \ldots b_{s} Y \in \mathcal{X} \otimes_{\mathcal{C}} \mathcal{Y}$ with $a_{i}, b_{j} \in \mathcal{C}$ is well-defined.

2.2. Tensor product over a 2-group. A more concise representation of the morphisms just defined is possible when the objects in the monoidal category are invertible with respect to the monoidal product. This is the case for our application in $\S 3$.

Definition 22. A 2-group is a monoidal category $\mathcal{C}$ such that for each $a \in \mathcal{C}$ there exists another object $a^{-1} \in \mathcal{C}$, and an isomorphism

$$
I_{a}: a \square a^{-1} \stackrel{\sim}{\longrightarrow} e
$$

where $e \in \mathcal{C}$ is the identity object.

Note that the object $a^{-1}$ is not necessarily unique, and neither is $I_{a}: a \square a^{-1} \rightarrow e$, even for a particular choice of $a^{-1}$. In the following, we will assume that the choices satisfy $\left(a^{-1}\right)^{-1}=a$.

The 2-group we will later apply the results of this section to is $\operatorname{Herm}_{1}\left(\mathcal{O}_{K}\right)$, the category of non-degenerate positive-definite rank-one hermitian modules over the ring of integers $\mathcal{O}_{K}$ of a $\mathrm{CM}$ field $K$. The isomorphism classes of $\operatorname{Herm}_{1}\left(\mathcal{O}_{K}\right)$ form the group of classes of hermitian forms, classically denoted $\mathfrak{C}(K)[26, \S 14.5]$.

Let $\mathcal{X}$ and $\mathcal{Y}$ be categories with a right and left action by a 2 -group $\mathcal{C}$, respectively. To prevent the congestion of symbols later on, we define the following auxiliary isomorphisms. For $X \in \mathcal{X}$, $Y \in \mathcal{Y}, a \in \mathcal{C}$, we have

$$
\mu_{X, a}:(X a) a^{-1} \stackrel{\sim}{\longrightarrow} X, \quad \mu_{a, Y}: a^{-1}(a Y) \stackrel{\sim}{\longrightarrow} Y,
$$

given by $\mu_{X, a}=\rho_{X} \circ\left(\mathbb{1}_{X} I_{a}\right) \circ \alpha_{X, a, a^{-1}}$, and $\mu_{a, Y}=\lambda_{Y} \circ\left(I_{a^{-1}} \mathbb{1}_{Y}\right) \circ \alpha_{Y, a^{-1}, a}^{-1}$. Note that these depend on a choice of $a^{-1}, I_{a}$ and $I_{a^{-1}}$.

For each $X \otimes Y \in \mathcal{X} \otimes \mathcal{Y}$, we also have an isomorphism

$$
\omega_{a, X, Y}: X a \otimes a^{-1} Y \stackrel{\sim}{\longrightarrow} X \otimes Y
$$

given by

$$
\omega_{a, X, Y}=\left(\mathbb{1}_{X} \otimes \mu_{a^{-1}, Y}\right) \circ \alpha_{X, a, a^{-1} Y}
$$


In other words, $\omega_{a, X, Y}$ is the diagonal morphism in the diagram

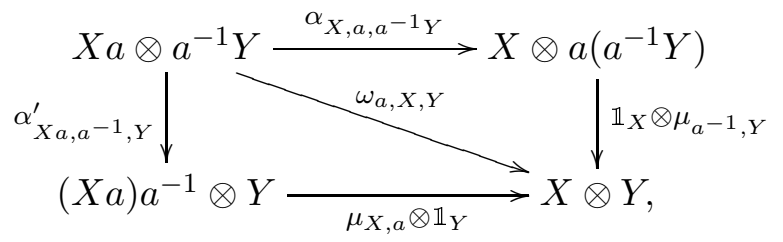

which commutes as a consequence of relations II and IV in Definition 21.

For each $\phi \otimes \psi: X \otimes Y \rightarrow X^{\prime} \otimes Y^{\prime}$ and $a \in \mathcal{C}$, we also have a diagram

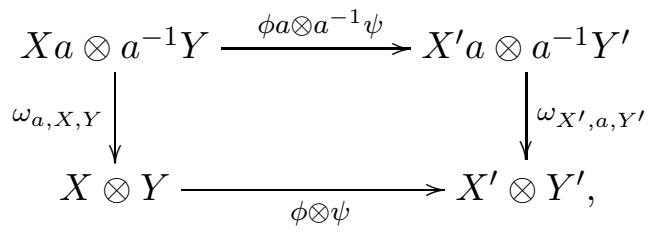

commuting as a consequence of relation II in Definition 21, along with functorial properties of the action of $\mathcal{C}$.

We will show that every morphism $X^{\prime} \otimes Y^{\prime} \rightarrow X \otimes Y$ in $\mathcal{X} \otimes_{\mathcal{C}} \mathcal{Y}$ can be written in the form $\omega_{a, X, Y} \circ(\phi \otimes \psi)$ for some $a \in \mathcal{C}, \phi \in \operatorname{Mor}(\mathcal{Y}), \psi \in \operatorname{Mor}(\mathcal{X})$. The following lemma is the essential reduction step in the proof.

Lemma 23. Suppose $\tau=\alpha_{2} \circ(\phi \otimes \psi) \circ \alpha_{1}$ is a morphism of $\mathcal{X} \otimes_{\mathcal{C}} \mathcal{Y}$, where $\phi \in \operatorname{Mor}(\mathcal{X}), \psi \in \operatorname{Mor}(\mathcal{Y})$ and $\alpha_{1}, \alpha_{2}$ are associator morphisms in $\mathcal{X} \otimes_{\mathcal{C}} \mathcal{Y}$. Then we can also write

$$
\tau=\left(\phi_{1} \otimes \psi_{1}\right) \circ \alpha \circ\left(\phi_{2} \otimes \psi_{2}\right),
$$

where $\alpha$ is another associator, and $\phi_{1}, \phi_{2} \in \operatorname{Mor}(\mathcal{X}), \psi_{1}, \psi_{2} \in \operatorname{Mor}(\mathcal{Y})$.

Proof. The associators $\alpha_{1}$ and $\alpha_{2}$ can each either have the form $\alpha_{X, a, Y}$ or its inverse $\alpha_{X, a, Y}^{\prime}$. Of the four possibilities, we look at the case where $\alpha_{1}$ and $\alpha_{2}$ have the form

$$
\alpha_{1}=\alpha_{X, a, Y}: X a \otimes Y \rightarrow X \otimes a Y, \quad \alpha_{2}=\alpha_{X^{\prime}, b, Y^{\prime}}: X^{\prime} b \otimes Y^{\prime} \rightarrow X^{\prime} \otimes b Y^{\prime},
$$

so that we have $\phi \otimes \psi: X \otimes a Y \rightarrow X^{\prime} b \otimes Y^{\prime}$. The other three cases are similar.

The claim then follows from the commutativity of the diagram

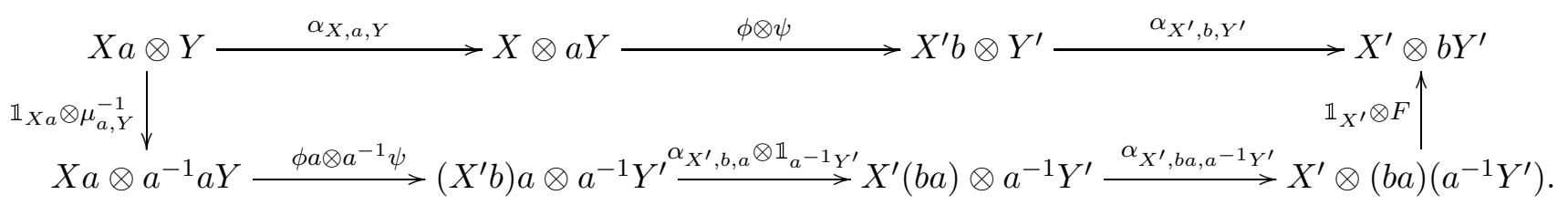

Here the morphism $F$ is the composition

$$
(b a)\left(a^{-1} Y^{\prime}\right) \stackrel{\alpha_{b a, a^{-1}, Y^{\prime}}^{\prime}}{\longrightarrow}\left((b a) a^{-1}\right) Y^{\prime} \stackrel{\left(\alpha_{b, a, a}-1\right) \mathbb{1}_{Y^{\prime}}}{\longrightarrow}\left(b\left(a a^{-1}\right)\right) Y^{\prime} \stackrel{\left(b I_{a}\right) \mathbb{1}_{Y^{\prime}}}{\longrightarrow}(b e) Y^{\prime} \stackrel{\rho_{b} \mathbb{1}_{Y^{\prime}}}{\longrightarrow} b Y^{\prime} .
$$

Checking that this diagram does indeed commute is straight-forward using the axioms of $\mathcal{X} \otimes_{\mathcal{C}} \mathcal{Y}$. In particular, one uses the triangle and pentagon relations and the naturality of associators.

Here is the main result on presentations of morphisms in $\mathcal{X} \otimes_{\mathcal{C}} \mathcal{Y}$.

Proposition 24. Let $\mathcal{X}$ (resp. $\mathcal{Y}$ ) be categories with a right (resp. left) action of a 2-group $\mathcal{C}$. Then every morphism $\tau: X \otimes Y \rightarrow X^{\prime} \otimes Y^{\prime}$ in $\mathcal{X} \otimes_{\mathcal{C}} \mathcal{Y}$ has a presentation as a composition

$$
X \otimes Y \stackrel{\phi \otimes \psi}{\longrightarrow} X^{\prime} a \otimes a^{-1} Y^{\prime} \stackrel{\omega_{a, X^{\prime}, Y^{\prime}}}{\longrightarrow} X^{\prime} \otimes Y^{\prime}
$$


for some object $a \in \mathcal{C}$, and morphisms $\phi, \psi$ in $\mathcal{X}, \mathcal{Y}$, respectively. Alternatively, $\tau$ can also be written as $\left(\phi^{\prime} \otimes \psi^{\prime}\right) \circ \omega_{a^{\prime}, X, Y}^{-1}$, for some other $\phi^{\prime}, \psi^{\prime}, a^{\prime}$.

Proof. By definition, a morphism $\tau$ of $\mathcal{X} \otimes_{\mathcal{C}} \mathcal{Y}$ is a string of symbols, each one of two types: associator morphisms $\alpha$, and tensor morphisms $\phi \otimes \psi$. Since a composition of two tensor morphisms is another tensor morphism, a word representing a general morphism can be reduced until the associators occurring in it are each separated by one tensor morphism (possibly the identity). Then as long as there remain at least two associators in the presentation of $\tau$, Lemma 23 applies, and each time the number of associators can be reduced by one. The process necessarily ends with a presentation of the form $\left(\phi_{1} \otimes \psi_{1}\right) \circ \alpha \circ\left(\phi_{2} \otimes \psi_{2}\right)$.

Then to finish the proof it suffices to show the claim for a morphism of the form $(\phi \otimes \psi) \circ \alpha$. Assuming $\alpha=\alpha_{X, a, Y}$ and $\phi \otimes \psi: X \otimes a Y \rightarrow X^{\prime} \otimes Y^{\prime}$, this follows from the commutativity of the diagram

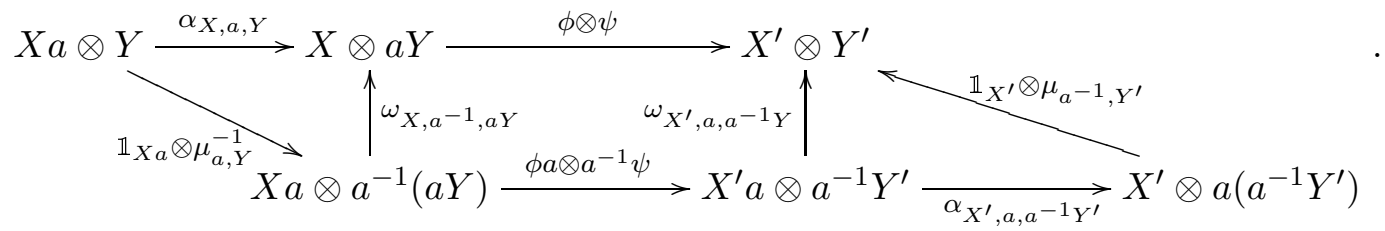

The commutativity of the two triangles on the left and right follow from instances of (2.4). The middle square is itself an instance of (2.5).

In case $\alpha$ is of the form $\alpha_{X, a, Y}^{\prime}$, a similar diagram gives a nearly identical presentation for $(\phi \otimes$ $\psi) \circ \alpha$, wherein $a$ is replaced by $a^{-1}$. The alternative presentation of $\tau$ in the form $\left(\phi^{\prime} \otimes \psi^{\prime}\right) \circ \omega_{a^{\prime}, X, Y}^{-1}$ results from yet other similar diagrams, with directions reversed.

Now we consider the case where $\mathcal{X}$ in $\mathcal{X} \otimes_{\mathcal{C}} \mathcal{Y}$ is a groupoid, and $\mathcal{Y}$ is fibred in groupoids over a base. We show that $\mathcal{X} \otimes_{\mathcal{C}} \mathcal{Y}$ is also fibred in groupoids, under some general conditions which we now define.

Definition 25. Let $p: \mathcal{Y} \rightarrow \mathcal{S}$ be a functor, and $\mathcal{C}$ a monoidal category acting on $\mathcal{Y}$ on the left. Then $\mathcal{C}$ is said to act fibrewise on $\mathcal{Y}$, if $p$ is a coequalizer in the diagram

$$
\mathcal{C} \times \mathcal{Y} \underset{p_{\mathcal{Y}}}{\stackrel{\square}{\longrightarrow}} \mathcal{Y} \stackrel{p}{\longrightarrow} \mathcal{S}
$$

and if $p$ sends the associators and unitors of the action of $\mathcal{C}$ to identity morphisms of $S$. Here denotes the action of $\mathcal{C}$, and $p \mathcal{y}$ is projection onto the second factor.

Definition 26. The left action of a monoidal category $\mathcal{C}$ on a category $\mathcal{Y}$ is said to be free on objects if whenever $a Y \simeq b Y$ in $\mathcal{Y}$ for some $Y \in \mathcal{Y}$, and $a, b \in \mathcal{C}$, then $a \simeq b$ in $\mathcal{C}$.

One can define a free action on the right analogously. These definitions appear essentially in [7] and [28, pp. 339-340], though neither spell out the behaviour on associators and unitors for a fibrewise action.

Recall that a category is called left-cancellative if all its morphisms are monic. We introduce the following relative version.

Definition 27. A category $\mathcal{Y}$ lying over $\mathcal{S}$ via $\pi: \mathcal{Y} \rightarrow \mathcal{S}$ is called left-cancellative over $\mathcal{S}$, if for any morphism $h: Z \rightarrow X$ in $\mathcal{Y}$, and any pair of morphisms $f: X \rightarrow Y$ and $g: X \rightarrow Y$ such that $\pi(f)=\pi(g)$, we have $f=g$ whenever $h \circ f=h \circ g$.

Lemma 28. A category fibred in groupoids $\mathcal{Y} \rightarrow \mathcal{S}$ is left-cancellative over $\mathcal{S}$.

Proof. Let $f: X \rightarrow Y, g: X \rightarrow Y$, and $h: Z \rightarrow Y$ be morphisms in $\mathcal{Y}$, and suppose $f \circ h=g \circ h$, with $f$ and $g$ lying over the same morphism in $\mathcal{S}$. Since $\mathcal{Y}$ is fibred in groupoids, every morphism 
of $\mathcal{Y}$ is cartesian. In particular $f$ is cartesian, hence there exists a unique morphism $\rho: X \rightarrow X$ lying over $\mathbb{1}_{S}$ such that $g=\rho \circ f$. Then we have $f \circ h=\rho \circ f \circ h$. Since $f \circ h$ is also cartesian, $\rho$

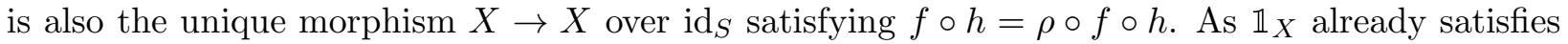
this, $\rho=\mathbb{1}_{X}$, therefore $g=f$.

Proposition 29. Let $\mathcal{X}$ be a groupoid on which a 2-group $\mathcal{C}$ acts on the right, and $p: \mathcal{Y} \rightarrow \mathcal{S}$ a category fibred in groupoids on which $\mathcal{C}$ acts on the left fibrewise and free on objects. Suppose furthermore that $\mathcal{X} \otimes_{\mathcal{C}} \mathcal{Y}$ is left-cancellative over $\mathcal{S}$. Then $\mathcal{X} \otimes_{\mathcal{C}} \mathcal{Y}$ is fibred in groupoids over $\mathcal{S}$ via $\pi: \mathcal{X} \otimes_{\mathcal{C}} \mathcal{Y} \rightarrow \mathcal{S}$ defined by

$$
\pi(X \otimes Y)=p(Y), \quad \pi(\phi \otimes \psi)=p(\psi), \quad \pi\left(\alpha_{X, a, Y}\right)=p\left(\mathbb{1}_{Y}\right) .
$$

Proof. Let $\alpha: T \rightarrow S$ be a morphism in $\mathcal{S}$, and $X \otimes Y$ an object in $\mathcal{X} \otimes_{\mathcal{C}} \mathcal{Y}$ over $S$. We must first show that $\alpha$ lifts to a morphism in $\mathcal{X} \otimes_{\mathcal{C}} \mathcal{Y}$ with target $X \otimes Y$. Now $Y \in \mathcal{Y}$ lies over $S \in \mathcal{S}$. Since $\mathcal{Y}$ is fibred in groupoids over $\mathcal{S}$, there exists an object $Y_{T} \in \mathcal{Y}$ over $T$ and a morphism $\psi: Y_{T} \rightarrow Y$ lifting $\alpha$. Therefore $\mathbb{1}_{X} \otimes \psi: X \otimes Y_{T} \rightarrow X \otimes Y$ is a morphism lifting $\alpha$ to $\mathcal{X} \otimes_{\mathcal{C}} \mathcal{Y}$.

Now suppose $f: X^{\prime} \otimes Y^{\prime} \rightarrow X \otimes Y$ lies over $\alpha: S^{\prime} \rightarrow S$, and $g: X^{\prime \prime} \otimes Y^{\prime \prime} \rightarrow X \otimes Y$ over $\beta: S^{\prime \prime} \rightarrow S$. Suppose $\gamma: S^{\prime} \rightarrow S^{\prime \prime}$ satisfies $\beta \circ \gamma=\alpha$. We must show there exists a unique morphism $h: X^{\prime} \otimes Y^{\prime} \rightarrow X^{\prime \prime} \otimes Y^{\prime \prime}$ lying over $\gamma$, such that $g \circ h=f$.

Using the alternate presentation of a morphism given in Proposition 24, we can write $f=$ $\left(\phi^{\prime} \otimes \psi^{\prime}\right) \circ \omega_{a}$ where $\omega_{a}: X^{\prime} \otimes Y^{\prime} \rightarrow X^{\prime} a \otimes a^{-1} Y^{\prime}$ is a canonical isomorphism for some $a \in \mathcal{C}$, and similarly $g=\left(\phi^{\prime \prime} \otimes \psi^{\prime \prime}\right) \circ \omega_{b}$ for some $b \in \mathcal{C}$. Then $\pi\left(\omega_{a}\right)=\mathbb{1}_{S^{\prime}}$ and $\pi\left(\omega_{b}\right)=\mathbb{1}_{S^{\prime \prime}}$ since $\mathcal{C}$ acts fibrewise on $\mathcal{Y}$, and so $\phi^{\prime} \otimes \psi^{\prime}$ and $\phi^{\prime \prime} \otimes \psi^{\prime \prime}$ are also lifts of $\alpha$ and $\beta$ to $\mathcal{X} \otimes_{\mathcal{C}} \mathcal{Y}$. Since $\omega_{a}$ and $\omega_{b}$ are isomorphisms, we have $g \circ h=f$ if and only if $\left(\phi^{\prime \prime} \otimes \psi^{\prime \prime}\right) \circ h^{\prime}=\left(\phi^{\prime} \otimes \psi^{\prime}\right)$, where $h^{\prime}=\omega_{b} \circ h \circ \omega_{a}^{-1}$. Therefore it's enough to assume $f=\phi^{\prime} \otimes \psi^{\prime}$ and $g=\phi^{\prime \prime} \otimes \psi^{\prime \prime}$, and show there's a unique $h$ lying over $\gamma$ such that $\left(\phi^{\prime \prime} \otimes \psi^{\prime \prime}\right) \circ h=\phi^{\prime} \otimes \psi^{\prime}$.

We have $p\left(\psi^{\prime}\right)=\pi\left(\phi^{\prime} \otimes \psi^{\prime}\right)=\alpha$, and $p\left(\psi^{\prime \prime}\right)=\pi\left(\phi^{\prime \prime} \otimes \psi^{\prime \prime}\right)=\beta$. As $\mathcal{Y}$ is fibred in groupoids over $\mathcal{S}$, there exists a unique lift $\eta: Y^{\prime} \rightarrow Y^{\prime \prime}$ of $\gamma$ to $\mathcal{Y}$, such that $\psi^{\prime \prime} \circ \eta=\psi^{\prime}$. Now, the maps $\phi^{\prime}$ and $\phi^{\prime \prime}$ are isomorphisms since $\mathcal{X}$ is a groupoid. Hence, setting $\xi=\phi^{\prime \prime-1} \circ \phi^{\prime}$, we obtain a map $\xi \otimes \eta: X^{\prime} \otimes Y^{\prime} \rightarrow X^{\prime \prime} \otimes Y^{\prime \prime}$ lifting $\gamma$, which satisfies the desired property $\left(\phi^{\prime \prime} \otimes \psi^{\prime \prime}\right) \circ(\xi \otimes \eta)=\phi^{\prime} \otimes \psi^{\prime}$. If $h: X^{\prime} \otimes Y^{\prime} \rightarrow X^{\prime \prime} \otimes Y^{\prime \prime}$ is any other lift such that $g \circ h=f=g \circ(\xi \otimes \eta)$ we have $h=\xi \otimes \eta$ since $\mathcal{X} \otimes_{\mathcal{C}} \mathcal{Y}$ is left-cancellative over $\mathcal{S}$. This shows the lift we constructed is unique, which finishes the proof that $\mathcal{X} \otimes \mathcal{C} \mathcal{Y}$ is fibred in groupoids over $\mathcal{S}$.

\section{Application to Moduli Spaces of Abelian Schemes.}

Using the results from $\S 1$, we apply the Serre tensor construction to certain moduli spaces of polarized abelian schemes related to PEL Shimura varieties. In the complex case, we show that we can construct all objects of the target moduli space in this way. Over a general base scheme, using deformation theory we show that all abelian schemes in the target family can be constructed étale locally on the base. These results are formulated as an equivalence of categories in the complex case, and an isomorphism of stacks in general.

3.1. The moduli space $\mathcal{M}_{\Phi}^{n}$. Let $K$ be a CM-field of degree $2 g$ over $\mathbb{Q}, \Phi$ a CM-type for $K$, and $n>0$ an integer. Let $L$ be the reflex field of $(K, \Phi)$. By $\mathcal{O}_{K}$, resp. $\mathcal{O}_{L}$, we denote the ring of integers of $K$, resp. $L$. We define a moduli space $\mathcal{M}_{\Phi}^{n}$ over $\operatorname{Spec} \mathcal{O}_{L}$ as follows.

Definition 30. For a locally noetherian scheme $S$ over $\operatorname{Spec} \mathcal{O}_{L}, \mathcal{M}_{\Phi}^{n}(S)$ is the category whose objects are triples $(A, \iota, \lambda)$ where:

- $A$ is an abelian scheme of relative dimension $n g$ over $S$.

- $\iota: \mathcal{O}_{K} \hookrightarrow \operatorname{End}_{S}(A)$ is an injective ring homomorphism taking complex conjugation on $\mathcal{O}_{K}$ to the Rosati involution on $\operatorname{End}_{S}(A)_{\mathbb{Q}}$.

- $\lambda: A \rightarrow A^{\vee}$ is an $\mathcal{O}_{K}$-linear principal polarization. 
In addition, the triple $(A, \iota, \lambda)$ is required to satisfy the following ideal condition. Let the ideal $J_{\Phi}$ be the kernel of the map

$$
\mathcal{O}_{K} \otimes \mathcal{O}_{L} \rightarrow \prod_{\phi \in \Phi} \mathbb{C}^{(\phi)}, \quad(\alpha \otimes \beta) \mapsto(\phi(\alpha) \cdot \beta)_{\phi}
$$

Here $\mathbb{C}^{(\phi)}$ denotes $\mathbb{C}$, considered as a $K$-algebra via $\phi: K \hookrightarrow \mathbb{C}$. We require that the action of $\mathcal{O}_{K} \otimes \mathcal{O}_{L}$ on $\operatorname{Lie}_{S}(A)$ satisfy

$$
J_{\Phi} \operatorname{Lie}_{S}(A)=0 .
$$

The morphisms of $\mathcal{M}_{\Phi}^{n}(S)$ are defined to be $\mathcal{O}_{K}$-linear isomorphisms of abelian schemes preserving the polarizations.

The functor $S \mapsto \mathcal{M}_{\Phi}^{n}(S)$ defines a category fibred in groupoids over the category $\mathrm{Sch}_{/ \mathcal{O}_{L}}$ of locally noetherian $\mathcal{O}_{L}$-schemes. It is representable by a Deligne-Mumford stack over Spec $\mathcal{O}_{L}$, which we also denote by $\mathcal{M}_{\Phi}^{n}$. When $n=1$, it is an integral model of the stack of principally polarized abelian varieties with CM by $(K, \Phi)$.

We first show $\mathcal{M}_{\Phi}^{n}$ is étale and proper over $\operatorname{Spec} \mathcal{O}_{L}$ (Theorem [35), generalizing results of B. Howard for the $n=1$ case [10, Theorem 2.1.3], as well as for $K$ quadratic imaginary [11, Proposition 2.1.2]. Our proof is essentially the same, using the deformation theory of abelian schemes. The key to adapting Howard's proof is the ideal condition, which we now discuss.

In order to obtain a well-behaved moduli space, one typically imposes restrictions on the action of $\iota(a)$ induced on $\operatorname{Lie}_{S}(A)$, for all $a \in \mathcal{O}_{K}$. For instance to obtain integral models of PEL Shimura varieties attached to unitary groups of a certain signature, one may impose a corresponding signature condition by prescribing the characteristic polynomial of $\iota(a)$ acting on $\operatorname{Lie}_{S}(A)$. For us, the relevant abelian schemes are those that are, over $\mathbb{C}$, isogenous to the $n$th power of a $\mathrm{CM}$ abelian variety of type $\Phi$. The corresponding signature condition is then

$$
\operatorname{charpoly}\left(\left.\iota(a)\right|_{\operatorname{Lie}_{S}(A)}, X\right)=\prod_{\phi \in \Phi}(X-\phi(a))^{n},
$$

where the right hand side is identified with its image under the map $\mathcal{O}_{L}[X] \rightarrow \mathcal{O}_{S}[X]$ induced by the structure morphism $S \rightarrow \operatorname{Spec} \mathcal{O}_{L}$.

However, over characteristic $p>0$ for $p$ ramified in $K$, the signature condition is not restrictive enough, since some embeddings $\phi \in \Phi$ may coincide. For example, let $K$ be quadratic imaginary, so that $L=K$ and $\Phi$ consists of a single embedding $\phi: K \hookrightarrow \mathbb{C}$. Let $S$ be a scheme over $\operatorname{Spec} \mathcal{O}_{K}$ of characteristic $p$, where $p$ is ramified in $K$. Then for any $a \in \mathcal{O}_{K}, a$ and $a^{\sigma}$ have the same image under $\mathcal{O}_{K} \rightarrow \mathcal{O}_{S}$, so that $\phi$ and $\phi \sigma$ are indistinguishable using the $\mathcal{O}_{K}$-action on $\mathcal{O}_{S}$, and the signature condition is always satisfied. This is a general defect of the signature condition that causes the moduli space to acquire vertical components over ramified primes $p$, and so fail to be flat over $\operatorname{Spec} \mathcal{O}_{K}$.

For $K$ quadratic imaginary, the wedge condition of G. Pappas [22, formulated using exterior powers of $\iota(a)$ acting on $\operatorname{Lie}_{S}(A)$, is one approach to fixing the defect over ramified primes. The resulting moduli spaces are expected to be flat in general, and this has been verified in important special cases. The wedge condition corresponding to (3.3) is simply $\iota(a)=\phi(a)$, i.e. that the two actions of $\mathcal{O}_{K}$ on $\operatorname{Lie}_{S}(A)$ should coincide. The resulting moduli space is then proper and smooth over $\operatorname{Spec} \mathcal{O}_{K}$, of relative dimension zero [11, 2.1.2]. However, it's not clear how to extend the wedge condition to the general $\mathrm{CM}$ case, since when $L \neq K$ there is no way to directly compare the actions of $\mathcal{O}_{K}$ and $\mathcal{O}_{L}$.

For all CM fields $K$, the ideal condition (3.2) fixes the above defect for the specific signature condition (3.3), which we are interested in. It is equivalent to (3.3) if $n=1$, or if $S$ has characteristic 0. In general it implies (3.3) (see Corollary 33). 
If $A \in \mathcal{M}_{\Phi}^{1}(S)$, so that it satisfies the ideal condition, and $M$ is a projective finitely presented $\mathcal{O}_{K}$-module, then $M \otimes_{\mathcal{O}_{K}} A$ also satisfies the ideal condition (by Lemma 3). Then if we want $\mathcal{M}_{\Phi}^{n}$ to consist of objects arising from the Serre construction (at least étale locally), the ideal condition on $\mathcal{M}_{\Phi}^{n}$ is necessary. On the other hand, we would like $\mathcal{M}_{\Phi}^{n}$ to have desirable properties such as flatness. We show that for this purpose the ideal condition is also sufficient, in the sense that $\mathcal{M}_{\Phi}^{n}$ as defined is étale and proper over $\operatorname{Spec} \mathcal{O}_{L}$ (Theorem 35). This fact is key to the proof of the main theorem in the last section.

First we expose some basic properties of the ideal $J_{\Phi}$. Following [10, let $\mathrm{Lie}_{\Phi}$ be defined by the exactness of the sequence of $\mathcal{O}_{K} \otimes \mathcal{O}_{L}$ modules

$$
0 \longrightarrow J_{\Phi} \longrightarrow \mathcal{O}_{K} \otimes \mathcal{O}_{L} \longrightarrow \operatorname{Lie}_{\Phi} \longrightarrow 0 \text {. }
$$

Since $\operatorname{Lie}_{\Phi}$ may be identified with the image of the map (3.1), it is a projective $\mathcal{O}_{L}$-module. Then the above sequence splits as $\mathcal{O}_{L}$-modules. In particular $J_{\Phi}$ is a direct $\mathcal{O}_{L}$-module summand of $\mathcal{O}_{K} \otimes \mathcal{O}_{L}$

Recall that $\sigma$ denotes complex conjugation on $\mathcal{O}_{K}$. We also use it to denote the induced $\mathcal{O}_{L}$-linear automorphism on $\mathcal{O}_{K} \otimes \mathcal{O}_{L}$.

Lemma 31. The ideal $J_{\Phi}$ satisfies the following properties:

(a) $J_{\Phi} J_{\Phi}^{\sigma}=J_{\Phi} \cap J_{\Phi}^{\sigma}=0$

(b) $J_{\Phi}$ is a projective $\mathcal{O}_{L}$-module of rank $g$ (where $2 g=[K: \mathbb{Q}]$ ).

(c) Suppose $T$ is a local $\mathcal{O}_{L}$-algebra, and $D$ is a free $\left(\mathcal{O}_{K} \otimes T\right)$-module of rank $n$. Then $J_{\Phi} D$ is the unique direct summand of $D$, as a $T$-module, that is $\mathcal{O}_{K}$-stable, has rank ng over $T$, and satisfies $J_{\Phi}(D / M)=0$.

Proof. For an embedding $\phi: \mathcal{O}_{K} \hookrightarrow \mathbb{C}$, let $\phi_{L}: \mathcal{O}_{K} \otimes \mathcal{O}_{L} \rightarrow \mathbb{C}$ denote the ring homomorphism $\alpha \otimes \beta \mapsto \phi(\alpha) \beta$. By definition, an element $x \in J_{\Phi}$ satisfies $\phi_{L}(x)=0$ for all $\phi \in \Phi$. Similarly, for $x \in J_{\Phi}^{\sigma}=J_{\Phi^{\sigma}}$ we have $\phi_{L}(x)=0$ for $\phi \in \Phi \sigma$. It follows that for $x \in J_{\Phi} \cap J_{\Phi}^{\sigma}$, we have $\phi_{L}(x)=0$ for all embeddings $\phi: \mathcal{O}_{K} \hookrightarrow \mathbb{C}$. But the map

$$
\mathcal{O}_{K} \otimes \mathcal{O}_{L} \rightarrow \prod_{\phi: \mathcal{O}_{K} \hookrightarrow \mathbb{C}} \mathbb{C}^{(\phi)}, \quad(\alpha \otimes \beta) \mapsto(\phi(\alpha) \beta)_{\phi}
$$

is injective, so $x=0$, which shows $J_{\Phi} \cap J_{\Phi}^{\sigma}=0$. Since $J_{\Phi} J_{\Phi}^{\sigma} \subseteq J_{\Phi} \cap J_{\Phi}^{\sigma}$, this proves (a).

For (b), we first note that $\mathcal{O}_{K} \otimes \mathcal{O}_{L}$ is free of rank $2 g$ over $\mathcal{O}_{L}$. Since $J_{\Phi}$ is an $\mathcal{O}_{L}$-submodule of $\mathcal{O}_{K} \otimes \mathcal{O}_{L}$, it is torsion-free, and hence projective. The rank can be verified over $\mathbb{C}$ by applying $\otimes_{\mathcal{O}_{L}} \mathbb{C}$ to (3.4), which becomes

$$
0 \longrightarrow \prod_{\phi \in \Phi \sigma} \mathbb{C}^{(\phi)} \longrightarrow \prod_{\phi: K \hookrightarrow \mathbb{C}} \mathbb{C}^{(\phi)} \longrightarrow \prod_{\phi \in \Phi} \mathbb{C}^{(\phi)} \longrightarrow 0 .
$$

For part (c), recall that $J_{\Phi}$ is a direct summand of $\mathcal{O}_{K} \otimes \mathcal{O}_{L}$ as an $\mathcal{O}_{L}$-module, since as such the exact sequence (3.4) is split. It follows that $J_{\Phi} D$ is a direct summand of $D$ as a $T$-module. As it is isomorphic to $J_{\Phi}\left(\mathcal{O}_{K} \otimes T\right)^{n} \cong\left(J_{\Phi} \otimes_{\mathcal{O}_{L}} T\right)^{n}$, by (b) it also has rank $n g$ over $T$, so it satisfies the properties mentioned in part (c). Now suppose $M$ is another such direct summand satisfying these properties. The condition $J_{\Phi}(D / M)=0$ implies $J_{\Phi} D \subset M$. It's an exercise in commutative algebra to show that in a free-module of finite rank, if one direct summand is contained in another one, and both have the same rank, they are equal. Hence $M=J_{\Phi} D$, showing uniqueness.

For an abelian scheme $A$ defined over $S$, we denote the first algebraic de Rham homology $H_{1}^{\mathrm{DR}}(A / S)=\mathcal{H}^{\mathrm{D}} \mathrm{m}_{\mathcal{O}_{S}}\left(H_{\mathrm{DR}}^{1}(A / S), \mathcal{O}_{S}\right)$ by $\mathbb{D}_{A}(S)$. There's a fundamental Hodge filtration, an exact sequence of locally free $\mathcal{O}_{S}$-modules

$$
0 \rightarrow \mathrm{Fil}^{1} \mathbb{D}_{A}(S) \rightarrow \mathbb{D}_{A}(S) \rightarrow \operatorname{Lie}_{S}(A) \rightarrow 0 .
$$


When $S=\operatorname{Spec} T$, the above may be identified with an exact sequence of projective $T$-modules by passing to global sections. We will often make this identification when $S$ is affine.

Proposition 32. Let $T$ be a local $\mathcal{O}_{L}$-algebra with a separable residue field $\mathbb{F}, S=\operatorname{Spec} T$, and $(A, \iota, \lambda) \in \mathcal{M}_{\Phi}^{n}(S)$. Then:

(a) $\mathbb{D}_{A}(S)$ is free of rank $n$ over $\mathcal{O}_{K} \otimes T$.

(b) The choice of an isomorphism $\left(\mathcal{O}_{K} \otimes T\right)^{n} \stackrel{\sim}{\longrightarrow} \mathbb{D}_{A}(S)$ leads to an isomorphism of short exact sequences

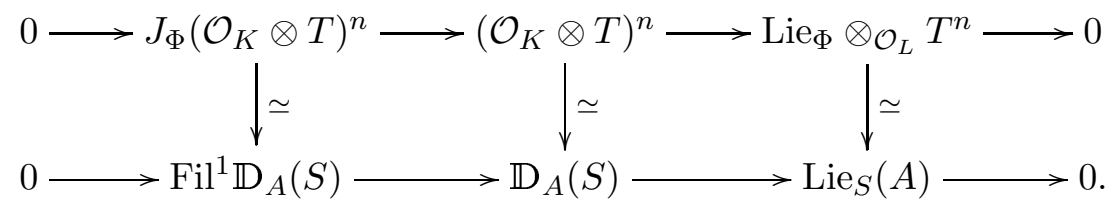

Proof. For (a), we first consider the case $T=\mathbb{F}$. If the characteristic of $\mathbb{F}$ is zero, then $\mathbb{D}_{A}(F)=$ $H_{1}^{\mathrm{DR}}(A / \mathbb{F})$ is free of rank $n$ over $\mathcal{O}_{K} \otimes \mathbb{F}$ by comparison with Betti homology. If the characteristic is $p>0$, one first shows that the covariant Dieudonné module $D(A)$ is free of rank $n$ over $\mathcal{O}_{K} \otimes W(\mathbb{F})$. This is proved in [23, Lemme 1.3], where it is stated in terms of $H_{c r i s}^{1}(A)$. The result then follows by $H_{1}^{\mathrm{DR}}(A / \mathbb{F}) \cong D(A) \otimes_{W(\mathbb{F})} \mathbb{F}$.

Now let $T$ be any local ring with residue field $\mathbb{F}$ and $S=\operatorname{Spec} T$. Let $A_{0}$ denote $A \otimes \mathbb{F}$. We have $\mathbb{D}_{A}(S) \otimes_{T} \mathbb{F} \cong \mathbb{D}_{A_{0}}(\mathbb{F}) \simeq\left(\mathcal{O}_{K} \otimes \mathbb{F}\right)^{n}$. Let $\left\{x_{1}, \cdots, x_{n}\right\}$ be the lift to $\mathbb{D}_{A}(S)$ of an $\left(\mathcal{O}_{K} \otimes \mathbb{F}\right)$-basis for $\mathbb{D}_{A_{0}}(\mathbb{F})$, and let $\left(\mathcal{O}_{K} \otimes T\right)^{n} \rightarrow \mathbb{D}_{A}(S)$ be the $\mathcal{O}_{K} \otimes T$-linear map sending $e_{i}$ to $x_{i}$. By Nakayama's lemma for the local ring $T$, this map is surjective. Let $K$ denote the kernel. Since $\mathbb{D}_{A}(S)$ is projective over $T$, we have $\left(\mathcal{O}_{K} \otimes T\right)^{n} \simeq K \oplus \mathbb{D}_{A}(S)$, which shows $K$ is also projective, hence free. Now applying $-\otimes_{T} \mathbb{F}$ to the isomorphism $\left(\mathcal{O}_{K} \otimes T\right)^{n} \simeq K \oplus \mathbb{D}_{A}(S)$ shows that $K \otimes_{T} \mathbb{F}=0$, which implies $K=0$ by considering rank. Thus the map $\left(\mathcal{O}_{K} \otimes T\right)^{n} \rightarrow \mathbb{D}_{A}(S)$ is also injective, hence an isomorphism.

For part (b), note that since $\left(\mathcal{O}_{K} \otimes T\right)^{n} \cong\left(\mathcal{O}_{K} \otimes \mathcal{O}_{L}\right) \otimes_{\mathcal{O}_{L}} T^{n}$, the first row can be obtained by tensoring (3.4) with $T^{n}$ over $\mathcal{O}_{L}$, so it is exact. The ideal condition $J_{\Phi} \operatorname{Lie}_{S}(A)=0$ and the exactness of the second row together imply that the composition $J_{\Phi}\left(\mathcal{O}_{K} \otimes T\right)^{n} \rightarrow\left(\mathcal{O}_{K} \otimes T\right)^{n} \rightarrow \mathbb{D}_{A}(S)$ lands in $\mathrm{Fil}^{1} \mathbb{D}_{A}(S)$, providing the map on the left. Exactness of the first row then provides the map on the right. As $\operatorname{Lie}_{S}(A)$ is a projective $T$-module of dimension $n g$, Fil ${ }^{1} \mathbb{D}_{A}(S)$ is a direct summand of $\mathbb{D}_{A}(S)$ satisfying the conditions in Lemma 31 (c), so it must coincide with $J_{\Phi} \mathbb{D}_{A}(S)$, which is the image of $J_{\Phi}\left(\mathcal{O}_{K} \otimes T\right)^{n}$ in $\mathbb{D}_{A}(S)$. Therefore the map on the left is also an isomorphism. Then since the vertical maps in the middle and the left are isomorphisms, so is the one on the right.

Corollary 33. Let $S$ be a scheme locally of finite type over $\operatorname{Spec} \mathcal{O}_{L}$, and $(A, \iota, \lambda) \in \mathcal{M}_{\Phi}^{n}(S)$. Then for each $a \in \mathcal{O}_{K}$,

$$
\operatorname{charpoly}\left(\left.\iota(a)\right|_{\operatorname{Lie}_{S}(A)}, X\right)=\prod_{\phi \in \Phi}(X-\phi(a))^{n} .
$$

Proof. The assertion is an identity of global sections of $\mathcal{O}_{S}[X]$, the given polynomial being identified with its image under $\mathcal{O}_{L}[X] \rightarrow \mathcal{O}_{S}[X]$. Since such an identity may be checked at the stalks of $\mathcal{O}_{S}[X]$, we may assume $S=\operatorname{Spec} T$, where $T$ is a local $\mathcal{O}_{L}$-algebra. Furthermore, since $S$ is locally of finite type over $\operatorname{Spec} \mathcal{O}_{L}$, the residue field of $T$ either has characteristic zero, or is a finite extension of the residue field of a closed point in $\operatorname{Spec} \mathcal{O}_{L}$. In either case, it is separable. Now by Lemma 32(b), $\operatorname{Lie}_{S}(A)$ is isomorphic to $\operatorname{Lie}_{\Phi} \otimes_{\mathcal{O}_{L}} T^{n}$ as an $\mathcal{O}_{K} \otimes T$-module. The characteristic polynomial of $a \in \mathcal{O}_{K}$ acting on $\operatorname{Lie}_{\Phi}$ can be seen to equal $\prod_{\phi \in \Phi}(X-\phi(a))$ after extending scalars to $\mathbb{C}$ and using the fact $\operatorname{Lie}_{\Phi} \otimes_{\mathcal{O}_{L}} \mathbb{C} \simeq \prod_{\phi \in \Phi} \mathbb{C}^{(\phi)}$. The image of the same polynomial under $\mathcal{O}_{L}[X] \rightarrow T[X]$ gives the characteristic polynomial of $a \in \mathcal{O}_{K}$ acting on $\operatorname{Lie}_{\Phi} \otimes_{\mathcal{O}_{L}} T$. Therefore $a \in \mathcal{O}_{K}$ acting on $\operatorname{Lie}_{S}(A) \simeq \operatorname{Lie} \otimes_{\mathcal{O}_{L}} T^{n} \cong\left(\operatorname{Lie} \otimes_{\mathcal{O}_{L}} T\right)^{n}$ has characteristic polynomial $\prod_{\phi \in \Phi}(X-\phi(a))^{n}$. 
We now turn to the deformation theory of abelian schemes to prove $\mathcal{M}_{\Phi}^{n}$ has the expected properties. Let us fix a point $y \in \operatorname{Spec} \mathcal{O}_{L}$. The completed étale local ring $\widehat{\mathcal{O}_{L, y}^{\text {sh }}}$ of $\operatorname{Spec} \mathcal{O}_{L}$ at $y$ is the completion of the ring of integers of the maximal unramified extension of $\mathcal{O}_{L, y}$. Following the notation of [10], we denote it by $W_{\Phi}$. Its residue field, a separable closure of $k(y)$, will be denoted $\mathbb{F}$. Let $\mathcal{C}_{\Phi}$ be the category of complete local noetherian $W_{\Phi}$-algebras with residue field $\mathbb{F}$, and $\mathcal{A}_{\Phi}$ its subcategory of artinian rings. The relevant facts from deformation theory of abelian schemes [14, Ch. 2] are summarized as follows.

Let $T^{\prime} \rightarrow T$ be a surjection in $\mathcal{C}_{\Phi}$ with kernel $I$ satisfying $I^{2}=0$. Set $S=\operatorname{Spec} T$ and $S^{\prime}=$ Spec $T^{\prime}$. An abelian scheme $A / S$ always lifts to some abelian scheme $A^{\prime} / S^{\prime}$, and for any such $A^{\prime}$ there's a canonical isomorphism $\mathbb{D}_{A^{\prime}}\left(S^{\prime}\right) \otimes_{T^{\prime}} T \cong \mathbb{D}_{A}(S)$. Furthermore, the $T^{\prime}$-module $\mathbb{D}_{A^{\prime}}\left(S^{\prime}\right)$ is up to canonical isomorphism independent of the choice of the lift. For convenience we hide away the canonical isomorphisms, erasing $A^{\prime}$ from the notation and writing $\widetilde{\mathbb{D}}_{A}\left(S^{\prime}\right)$ instead of $\mathbb{D}_{A^{\prime}}\left(S^{\prime}\right)$. The submodule Fil ${ }^{1} \mathbb{D}_{A^{\prime}}\left(S^{\prime}\right)$ of $\widetilde{\mathbb{D}}_{A}\left(S^{\prime}\right)$ on the other hand does depend on the choice of the lift, and determines it completely. More specifically, there is a bijection between lifts $A^{\prime} / S^{\prime}$ of $A / S$, and projective $T^{\prime}$-submodules $M$ of $\widetilde{\mathbb{D}}_{A}\left(S^{\prime}\right)$ such that $M \otimes_{T^{\prime}} T \cong \mathrm{Fil}^{1} \mathbb{D}_{A}(S)$ via the isomorphism $\widetilde{\mathbb{D}}_{A}\left(S^{\prime}\right) \otimes_{T^{\prime}} T \cong \mathbb{D}_{A}(S)$.

Let $A^{\prime} / S^{\prime}$ be a lift of $A / S$. For an element $\phi \in \operatorname{End}_{S}(A)$, the induced $T$-module endomorphism of $\mathbb{D}_{A}(S)$ lifts canonically to a morphism $\phi_{*}: \widetilde{\mathbb{D}}_{A}\left(S^{\prime}\right) \rightarrow \widetilde{\mathbb{D}}_{A}\left(S^{\prime}\right)$. Then $\phi$ lifts (uniquely) to an endomorphism of abelian schemes $\phi^{\prime}: A^{\prime} \rightarrow A^{\prime}$ if and only if $\phi_{*}$ leaves the corresponding $T^{\prime}$ submodule Fil $\mathbb{D}_{A^{\prime}}\left(S^{\prime}\right)$ invariant. In particular, an $\mathcal{O}_{K}$-action $\iota: \mathcal{O}_{K} \hookrightarrow \operatorname{End}_{S}(A)$ lifts (uniquely) to an $\mathcal{O}_{K}$-action $\iota^{\prime}$ on $A^{\prime}$ if and only if $\mathrm{Fil}^{1} \mathbb{D}_{A^{\prime}}\left(S^{\prime}\right)$ is an $\mathcal{O}_{K}$-submodule of $\widetilde{\mathbb{D}}_{A}\left(S^{\prime}\right)$.

A polarization $\lambda: A \rightarrow A^{\vee}$ induces a symplectic pairing $\langle,\rangle_{\lambda}$ on $\mathbb{D}_{A}(S)$ which lifts to a pairing on $\widetilde{\mathbb{D}}_{A}\left(S^{\prime}\right)$, denoted $\langle,\rangle_{\lambda}^{\prime}$. Given a lift $A^{\prime} / S^{\prime}$ of $A / S, \lambda$ lifts (uniquely) to a map $\lambda^{\prime}: A^{\prime} \rightarrow A^{\prime \vee}$ if and only if the submodule $\mathrm{Fil}^{1} \mathbb{D}_{S^{\prime}}\left(A^{\prime}\right)$ of $\widetilde{\mathbb{D}}_{A}\left(S^{\prime}\right)$ is totally isotropic for $\langle,\rangle_{\lambda}^{\prime}$. In that case $\lambda^{\prime}$ is a polarization for $A^{\prime}$, and it is principal if $\lambda$ is. If $A$ has an $\mathcal{O}_{K^{-}}$action that lifts to $A^{\prime}$ and $\lambda$ is $\mathcal{O}_{K}$-linear, so is $\lambda^{\prime}$. In addition, the Rosati involution induced by $\lambda$ corresponds to the adjoint for the pairing $\langle,\rangle_{\lambda}^{\prime}$, so that $\langle a x, y\rangle_{\lambda}^{\prime}=\left\langle x, a^{\sigma} y\right\rangle_{\lambda}^{\prime}$ for all $x, y \in \widetilde{\mathbb{D}}_{A}\left(S^{\prime}\right), a \in \mathcal{O}_{K}$.

Putting the above facts together, we see that lifting a triple $(A, \iota, \lambda)$ over $S$ to $\left(A^{\prime}, \iota^{\prime}, \lambda^{\prime}\right)$ over $S^{\prime}$ is equivalent to lifting the Hodge filtration $\mathrm{Fil}^{1} \mathbb{D}_{A}(S)$ of $A$ to an $\mathcal{O}_{K}$-stable projective $T^{\prime}$-submodule of $\widetilde{\mathbb{D}}_{A}\left(S^{\prime}\right)$ that is totally isotropic with respect to the pairing $\langle,\rangle_{\lambda}^{\prime}$. Such a lift satisfies the ideal condition if and only if $J_{\Phi} \widetilde{\mathbb{D}}_{A}\left(S^{\prime}\right) \subseteq \operatorname{Fil}^{1} \mathbb{D}_{A^{\prime}}\left(S^{\prime}\right)$.

Proposition 34. Every object in $\mathcal{M}_{\Phi}^{n}(\mathbb{F})$ lifts uniquely to $\mathcal{M}_{\Phi}^{n}(T)$, for all $T \in \mathcal{C}_{\Phi}$.

Proof. Every $T$ in $\mathcal{C}_{\Phi}$ is an inverse limit of its quotients, which are artinian rings in $\mathcal{A}_{\Phi} \subset \mathcal{C}_{\Phi}$. Then it's enough to show the claim for $T \in \mathcal{A}_{\Phi}$, since $\mathcal{M}_{\Phi}^{n}$ is an algebraic stack for which formal deformations are effective. Each such artinian ring has a surjective map to $\mathbb{F}$ which is a composition of finitely many surjections in $\mathcal{A}_{\Phi}$, with square-zero kernels. Therefore it suffices to show that for a surjective map $T^{\prime} \rightarrow T$ in $\mathcal{A}_{\Phi}$ having square-zero kernel, with $S=\operatorname{Spec} T$ and $S^{\prime}=\operatorname{Spec} T^{\prime}$, every object $(A, \iota, \lambda)$ of $\mathcal{M}_{\Phi}^{n}(S)$ lifts uniquely to an object of $\mathcal{M}_{\Phi}^{n}\left(S^{\prime}\right)$. For such an object $(A, \iota, \lambda)$, we have the Hodge filtration of projective $T$-modules (3.5). By Proposition $32, \mathrm{Fil}^{1} \mathbb{D}_{A}(S)$ is the $T$-submodule $J_{\Phi} \mathbb{D}_{A}(S)$ of $\mathbb{D}_{A}(S)$. For the same reason, any lift $\left(A^{\prime}, \iota^{\prime}, \lambda^{\prime}\right)$ of $(A, \iota, \lambda)$ to $S^{\prime}$, if such exists, would have Fil ${ }^{1} \mathbb{D}_{A^{\prime}}\left(S^{\prime}\right)=J_{\Phi} \mathbb{D}_{A^{\prime}}\left(S^{\prime}\right)=J_{\Phi} \widetilde{D}_{A}\left(S^{\prime}\right)$, and would therefore be unique, by the deformation theory outlined above.

Now we claim $M=J_{\Phi} \widetilde{\mathbb{D}}_{A}\left(S^{\prime}\right) \subset \widetilde{\mathbb{D}}_{A}\left(S^{\prime}\right)$ does indeed correspond to a lift of $(A, \iota, \lambda)$ to $S^{\prime}$. It lifts the Hodge filtration since $M \otimes_{T^{\prime}} T=J_{\Phi} \widetilde{\mathbb{D}}_{A}\left(S^{\prime}\right) \otimes_{T^{\prime}} T \cong J_{\Phi} \mathbb{D}_{A}(S)$, which is equal to $\mathrm{Fil}^{1} \mathbb{D}_{A}(S)$ as we have just noted. Since $M$ is $\mathcal{O}_{K}$-stable, the pair $(A, \iota)$ lifts (uniquely) to a pair $\left(A^{\prime}, \iota^{\prime}\right)$. Since $J_{\Phi} \widetilde{\mathbb{D}}_{A}\left(S^{\prime}\right) \subseteq M$, the pair $\left(A^{\prime}, \iota^{\prime}\right)$ satisfies the ideal condition. It remains to show that $M$ is totally 
isotropic for $\langle,\rangle_{\lambda}^{\prime}$. For $x, y \in \widetilde{\mathbb{D}}_{A}\left(S^{\prime}\right)$ and $r, s \in J_{\Phi}$, we have

$$
\langle r x, s y\rangle_{\lambda}^{\prime}=\left\langle s^{\sigma} r x, y\right\rangle_{\lambda}^{\prime}=0,
$$

since $s^{\sigma} r=0$ by Lemma 31(a).

Theorem 35. The stack $\mathcal{M}_{\Phi}^{n}$ is étale and proper over $\operatorname{Spec} \mathcal{O}_{L}$.

Proof. Let $\mathbb{F}$ be a separably closed field, and $\bar{x}: \operatorname{Spec} \mathbb{F} \rightarrow \mathcal{M}_{\Phi}^{n}$ a geometric point of $\mathcal{M}_{\Phi}^{n}$. Let $\bar{y}: \operatorname{Spec} \mathbb{F} \rightarrow \operatorname{Spec} \mathcal{O}_{L}$ be the underlying geometric point, with image $y \in \operatorname{Spec} \mathcal{O}_{L}$. Fix a surjective étale morphism $M \rightarrow \mathcal{M}$ from a scheme $M$. Then $\bar{x}$ lifts to a map Spec $\mathbb{F} \rightarrow M$ with image $x \in M$ lying over $y \in \operatorname{Spec}\left(\mathcal{O}_{L}\right)$, and $M \rightarrow \operatorname{Spec}\left(\mathcal{O}_{L}\right)$ induces a map of étale local rings $\mathcal{O}_{L, y}^{\mathrm{sh}} \rightarrow \mathcal{O}_{M, x}^{\mathrm{sh}}$. To show $\mathcal{M}_{\Phi}^{n} \rightarrow \operatorname{Spec}\left(\mathcal{O}_{L}\right)$ is étale, it's enough to show the induced map on completions

$$
\widehat{\mathcal{O}_{L, y}^{\mathrm{sh}}} \rightarrow \widehat{\mathcal{O}_{M, x}^{\mathrm{sh}}}
$$

is an isomorphism. The ring $\widehat{\mathcal{O}_{L, y}^{\text {sh }}}$, which is the completion of the ring of integers of the maximal unramified extension of $\mathcal{O}_{L, y}$, will be denoted $W_{\Phi}$ as before. The $\operatorname{ring} \widehat{\mathcal{O}_{M, x}^{\mathrm{sh}}}$, which is up to isomorphism independent of the choice of $M \rightarrow \mathcal{M}_{\Phi}^{n}$, will be denoted $R_{\mathcal{M}}$.

The geometric point $\bar{x}$ lifts uniquely to a map $\operatorname{Spec} \mathbb{F} \rightarrow \operatorname{Spec} R_{\mathcal{M}}$, whose image lies over $x \in M$. The image of the corresponding ring homomorphism $R_{\mathcal{M}} \rightarrow \mathbb{F}$ is the separable closure $\mathbb{F}^{\prime}$ of the residue field $k(x)$ of $\mathcal{O}_{M, x}$ in $\mathbb{F}$. Then Spec $\mathbb{F} \rightarrow$ Spec $R_{\mathcal{M}}$ factors uniquely through Spec $\mathbb{F} \rightarrow$ Spec $\mathbb{F}^{\prime}$, so it is harmless to assume $\mathbb{F}$ itself is the residue field of $R_{\mathcal{M}}$. Then since $M \rightarrow \operatorname{Spec} \mathcal{O}_{L}$ is locally of finite type, $\mathbb{F}$ is also the residue field of $W_{\Phi}$.

Let $\mathcal{C}_{\Phi}$ be the category of complete local noetherian $W_{\Phi}$-algebras with residue field $\mathbb{F}$. If $\left(A_{x}, \iota_{x}, \lambda_{x}\right) \in \mathcal{M}_{\Phi}^{n}(\mathbb{F})$ corresponds to $\bar{x}: \operatorname{Spec} \mathbb{F} \rightarrow \mathcal{M}_{\Phi}^{n}$, then for any $T \in \mathcal{C}_{\Phi}$, the set $\operatorname{Hom}_{W_{\Phi}}\left(R_{\mathcal{M}}, T\right)$ corresponds to lifts of $\left(A_{x}, \iota_{x}, \lambda_{x}\right)$ to $\mathcal{M}_{\Phi}^{n}(T)$. By Proposition 34, there is a unique such lift for every $T$, hence a unique morphism $R_{\mathcal{M}} \rightarrow T$ of $W_{\Phi}$-algebras. In other words, $R_{\mathcal{M}}$ is an initial object of $\mathcal{C}_{\Phi}$. Since $W_{\Phi}$ is also an initial object, the map $W_{\Phi} \rightarrow R_{\mathcal{M}}$ must be an isomorphism. This proves $\mathcal{M}_{\Phi}^{n} \rightarrow \operatorname{Spec} \mathcal{O}_{L}$ is étale.

The fact that $\mathcal{M}_{\Phi}^{n}$ is proper over $\operatorname{Spec} \mathcal{O}_{L}$ follows from the valuative criterion of properness. The proof is identical to the quadratic imaginary case in [10, Proposition 2.1.2].

Next we begin the systematic construction of the morphisms and objects of $\mathcal{M}_{\Phi}^{n}$.

3.2. Serre construction of $\mathcal{M}_{\Phi}^{n}$ : the morphisms. Let $(K, \Phi)$ be, as in the previous section, a CM-field of degree $2 g$ over $\mathbb{Q}$, and set $R=\mathcal{O}_{K}$. Let $\operatorname{Herm}_{n}(R)$ denote the category of pairs $(M, h)$ consisting of a projective finitely presented $R$-module $M$ of rank $n$, and a non-degenerate positive-definite $R$-hermitian form $h: M \rightarrow M^{\vee}$. It follows from Theorem 17 that given an object $(A, \iota, \lambda)$ of $\mathcal{M}_{\Phi}^{1}(S)$, and another $(M, h)$ of $\operatorname{Herm}_{n}(R)$, the triple

$$
\left(M \otimes_{R} A, \iota \otimes \mathbb{1}_{A}, h \otimes \lambda\right)
$$

is a well-defined object of $\mathcal{M}_{\Phi}^{n}(S)$. We will denote it by

$$
(M, h) \otimes(A, \iota, \lambda) .
$$

It follows that the 2-group $\operatorname{Herm}_{1}(R)$ acts on $\mathcal{M}_{\Phi}^{1}(S)$ on the left via the Serre construction. It also acts on $\operatorname{Herm}_{n}(R)$ on the right via ordinary tensor product. Thus, as described in $\S 2$, we can form the the tensor product category

$$
\operatorname{Herm}_{n}(R) \otimes_{\operatorname{Herm}_{1}(R)} \mathcal{M}_{\Phi}^{1}(S) .
$$

To avoid confusion with the Serre construction, we denote the objects of this category by

$$
(M, h) \otimes(A, \iota, \lambda) .
$$


Likewise, we denote the pure tensor morphisms in the same category by $f \otimes \phi$. There is then a functor

$$
\Sigma_{S}: \operatorname{Herm}_{n}(R) \otimes_{\operatorname{Herm}_{1}(R)} \mathcal{M}_{\Phi}^{1}(S) \longrightarrow \mathcal{M}_{\Phi}^{n}(S)
$$

given on objects by

$$
\Sigma_{S}:(M, h) \otimes(A, \iota, \lambda) \mapsto(M, h) \otimes(A, \iota, \lambda),
$$

sending morphisms $f \otimes \phi$ to $f \otimes \phi$, and mapping associator isomorphisms to their counterparts. The purpose of most of this section is to prove the following.

Proposition 36. The functor $\Sigma_{S}: \operatorname{Herm}_{n}(R) \otimes_{\operatorname{Herm}_{1}(R)} \mathcal{M}_{\Phi}^{1}(S) \longrightarrow \mathcal{M}_{\Phi}^{n}(S)$ is fully faithful.

We will first characterize the morphisms in $\mathcal{M}_{\Phi}^{n}$ that are in the image of the functor $\Sigma_{S}$, and then compare our result with the description given in $\S 2$ of the morphisms of $\operatorname{Herm}_{n}(R) \otimes_{\operatorname{Herm}_{1}(R)} \mathcal{M}_{\Phi}^{1}(S)$.

For general abelian schemes $A$ and $B$ over a connected base $S$, and a point $s \in S$, by the rigidity lemma of [20, Ch. 6], the map $\operatorname{Hom}_{S}(A, B) \rightarrow \operatorname{Hom}_{k(s)}\left(A_{s}, B_{s}\right)$ given by base change to the fibre at $s$ is injective. For $R$-linear maps of abelian schemes in $\mathcal{M}_{\Phi}^{1}(S)$, we have the following.

Theorem 37. Let $(A, \iota, \lambda),(B, \jmath, \mu) \in \mathcal{M}_{\Phi}^{1}(S)$ with $\operatorname{Hom}_{R}(A, B) \neq 0$. Then for any morphism $T \rightarrow$ $S$ of connected locally noetherian $\mathcal{O}_{L}$-schemes, the map $\operatorname{Hom}_{R}(A, B) \rightarrow \operatorname{Hom}_{R}\left(A_{T}, B_{T}\right)$ induced by base change is a bijection.

Proof. By the rigidity lemma of [20, §6.1], the map in question is injective, so it's enough to show surjection.

First we show the hom sheaf $\mathcal{H}=\underline{\operatorname{Hom}}_{R}(A, B)$ is representable by an étale scheme over $S$. It is well-known that $\mathcal{H}$ is representable by a scheme locally of finite type over $S$ (e.g. [9, §6], or [2, proof of 1.4.4.5]). $\mathcal{H} / S$ is locally of finite presentation, since it's locally of finite type and $S$ is locally noetherian. Then to show $\mathcal{H}$ is étale over $S$ it's enough to show it's formally étale.

Let $T_{0} \hookrightarrow T$ be a closed immersion of $S$-schemes defined by a square-zero sheaf of $\mathcal{O}_{T}$-ideals. Let $u: A_{T_{0}} \rightarrow B_{T_{0}}$ be in $\mathcal{H}\left(T_{0}\right)$. We must show $u$ lifts to an $R$-linear morphism $\widetilde{u}: A_{T} \rightarrow B_{T}$. Uniqueness is automatic by infinitesimal rigidity [14, Lemma 2.2.2.1]. For any such $u$, the induced map $u_{*}: \mathbb{D}_{A}\left(T_{0}\right) \rightarrow \mathbb{D}_{B}\left(T_{0}\right)$ on de Rham homology lifts to a $\widetilde{u}_{*}: \widetilde{\mathbb{D}}_{A}(T) \rightarrow \widetilde{\mathbb{D}}_{B}(T)$ of $\mathcal{O}_{T}$-modules [14, 2.1.6.4]. The morphism $u$ lifts to a $\widetilde{u}: A_{T} \rightarrow B_{T}$ if and only if $\widetilde{u}_{*}$ respects the Hodge filtrations [14, 2.1.6.9]. By Proposition [32, we must show $\widetilde{u}_{*}\left(J_{\Phi} \widetilde{\mathbb{D}}_{A}(T)\right) \subset J_{\Phi} \widetilde{\mathbb{D}}_{B}(T)$. This follows from $J_{\Phi} \subset R \otimes \mathcal{O}_{L}$, and the fact that $\widetilde{u}_{*}$ is $R \otimes \mathcal{O}_{L}$-linear. Thus $\mathcal{H} \rightarrow S$ is formally étale, hence étale. It is also surjective since $\mathcal{H}$ as an $S$-group scheme admits a section.

The valuative criterion of properness implies, as in [9, Corollary 6.9], that every connected component of $\mathcal{H}$ is proper, and hence finite étale, over $S$.

Let $s \rightarrow S$ be a fixed geometric point. Applying the equivalence of [19, Theorem I.5.3] to every connected component of $\mathcal{H}$ equips $\mathcal{H}_{s}$ with an $R$-linear action of $\pi_{1}^{\text {et }}(S, s)$. The fundamental group acts trivially on the image of $\operatorname{Hom}(A, B) \rightarrow \mathcal{H}_{s}$, which has finite index since $\operatorname{Hom}(A, B)$ and $\mathcal{H}_{s}$ are rank-one projective $R$-modules. It follows that the action is trivial. Then by [19, Theorem I.5.3], each connected component of $\mathcal{H}$ is a constant $S$-scheme, isomorphic to $S$ itself.

Hence $\mathcal{H}$ is a disjoint union of copies of $S$, from which the theorem follows.

Let $S \in \operatorname{Sch} / \mathcal{O}_{L}$ be connected, and suppose $(A, \iota, \lambda),(B, \jmath, \mu)$ are in $\mathcal{M}_{\Phi}^{1}(S)$. Any $f \in \operatorname{Hom}_{R}(A, B)$ has a Rosati dual $f^{\prime} \in \operatorname{Hom}_{R}(B, A)$ defined by $f^{\prime}=\lambda^{-1} \circ f^{\vee} \circ \mu$.

Proposition 38. Let $(A, \iota, \lambda),(B, \jmath, \mu) \in \mathcal{M}_{\Phi}^{1}(S)$, with $\operatorname{Hom}_{R}(A, B) \neq 0$. Let $\operatorname{Hom}_{R}(B, A) \otimes_{R} B$ be equipped with the induced $R$-action. Then:

(a) The canonical map $\operatorname{Hom}_{R}(B, A) \otimes_{R} B \rightarrow A$ is an R-linear isomorphism. 
(b) The map

$$
\operatorname{Hom}_{R}(B, A) \otimes_{R} \operatorname{Hom}_{R}(A, B) \rightarrow \operatorname{End}(A), \quad f \otimes g \mapsto f \circ g
$$

is an isomorphism of $R$-modules.

(c) The map

$$
\operatorname{Hom}_{R}(A, B) \times \operatorname{Hom}_{R}(A, B) \rightarrow R, \quad(f, g) \mapsto \iota^{-1}\left(g^{\prime} \circ f\right)
$$

is a positive-definite non-degenerate hermitian form.

Proof. The map in (a) is an isogeny, so its kernel is a finite group scheme. Its triviality can then be checked on geometric points $\operatorname{Spec}(k) \rightarrow S$. By Proposition 34 one can reduce to characteristic zero, and even assume $k=\mathbb{C}$. In that case we have $B(\mathbb{C}) \simeq \mathbb{C}^{g} / \Phi(\mathfrak{b})$, where $\mathfrak{b}$ is a fractional ideal of $K$ and $\Phi(\mathfrak{b})$ is the image of

$$
\mathfrak{b} \rightarrow \mathbb{C}^{g}, \quad b \mapsto\left(\phi_{1}(b), \phi_{2}(b), \cdots, \phi_{g}(b)\right),
$$

with $\left(\phi_{1}, \cdots, \phi_{g}\right)$ a fixed ordering of $\Phi$. Similarly $A(\mathbb{C}) \simeq \mathbb{C}^{g} / \Phi(\mathfrak{a})$ for a fractional ideal $\mathfrak{a}$, and so $\operatorname{Hom}_{R}(B, A) \cong \mathfrak{b}^{-1} \mathfrak{a}$. The canonical map $\operatorname{Hom}_{R}(B, A) \otimes_{R} B \rightarrow A$ is then induced by the multiplication map $\mathfrak{b}^{-1} \mathfrak{a} \otimes_{R} \mathfrak{b} \rightarrow \mathfrak{a}$, which is clearly an isomorphism. This proves (a).

For (b), the given map is the composition of the following canonical isomorphisms

$$
\begin{aligned}
\operatorname{Hom}_{R}(B, A) \otimes_{R} \operatorname{Hom}_{R}(A, B) & \cong \operatorname{Hom}_{R}\left(R, \operatorname{Hom}_{R}(B, A)\right) \otimes_{R} \operatorname{Hom}_{R}(A, B) & & \\
& \cong \operatorname{Hom}_{R}\left(R \otimes_{R} A, \operatorname{Hom}_{R}(B, A) \otimes_{R} B\right) & & \text { (by Prop. 2(c)) } \\
& \cong \operatorname{Hom}_{R}(A, A)=\operatorname{End}_{R}(A) & & \text { (using (a)) }
\end{aligned}
$$

It's clear that the given pairing in (c) is hermitian. For non-zero $f \in \operatorname{Hom}_{R}(A, B)$, we have $\lambda \circ\left(f^{\prime} \circ f\right)=f^{\vee} \circ \mu \circ f$ which is a polarization on $A$, so $\iota^{-1}\left(f^{\prime} \circ f\right)$ is totally positive by Corollary 11. The bilinear map corresponds to the linear map in (b) up to composition with the Rosati dual and the isomorphism $\iota$. Its non-degeneracy is then equivalent to the isomorphism in (b).

The following lemma is a step towards characterizing the morphisms of $\mathcal{M}_{\Phi}^{n}(S)$.

Lemma 39. Let $(M, h),(N, k) \in \operatorname{Herm}_{n}(R)$, and $(A, \iota, \lambda),(B, \jmath, \mu) \in \mathcal{M}_{\Phi}^{1}(S)$. Suppose that $f: M \rightarrow N$ and $\phi: A \rightarrow B$ are $R$-linear homomorphisms, such that $f \otimes \phi$ is a morphism in $\mathcal{M}_{\Phi}^{n}(S)$. Then there exists a totally real unit $r \in R^{\times}$such that

$$
f:(M, h) \rightarrow(N, r \cdot k) \text { and } \phi:(A, \iota, \lambda) \rightarrow\left(B, \jmath, r^{-1} \cdot \mu\right)
$$

are morphisms in $\operatorname{Herm}_{n}(R)$ and $\mathcal{M}_{\Phi}^{1}(S)$, respectively.

Proof. Since $f \otimes \phi$ is a morphism in $\mathcal{M}_{\Phi}^{n}(S)$, we have

$h \otimes \lambda=(f \otimes \phi)^{\vee} \circ(k \otimes \mu) \circ(f \otimes \phi)=\left(f^{\vee} \otimes \phi^{\vee}\right) \circ(k \otimes \mu) \circ(f \otimes \phi)=\left(f^{\vee} \circ k \circ f\right) \otimes\left(\phi^{\vee} \circ \mu \circ \phi\right)$.

As $\lambda$ is principal, it generates the $R$-module $\operatorname{Hom}_{R}\left(A, A^{\vee}\right)$, so for some $r \in R$ we have

$$
\phi^{\vee} \circ \mu \circ \phi=r \cdot \lambda, \quad h=r \cdot\left(f^{\vee} \circ k \circ f\right) .
$$

Now we have

$$
M^{\vee} \supseteq \operatorname{im}\left(f^{\vee} \circ k \circ f\right) \supseteq r \cdot \operatorname{im}\left(f^{\vee} \circ k \circ f\right)=\operatorname{im}(h),
$$

where $\operatorname{im}(\cdot)$ denotes the image of a map. But since $h$ as an isomorphism is surjective, we have $\operatorname{im}(h)=M^{\vee}$, therefore

$$
M^{\vee}=\operatorname{im}(h)=r \cdot \operatorname{im}\left(f^{\vee} \circ k \circ f\right)=r M^{\vee} .
$$

By Nakayama's lemma, $M^{\vee}=r M^{\vee}$ implies $r$ is a unit in $R$. Now $\phi: A \rightarrow B$ is an isogeny, so $\phi^{\vee} \circ \mu \circ \phi=r \cdot \lambda=\lambda \circ \iota(r)$ is a polarization on $A$. By Corollary 11, $r$ must be totally positive. 
On the other hand, since $r$ is a unit and $\lambda$ is an isomorphism, so is $\lambda \circ \iota(r)=\phi^{\vee} \circ \mu \circ \phi$. In particular, the isogeny $\phi$ is injective, and is therefore an isomorphism. The map $R \rightarrow \operatorname{Hom}_{R}(A, B), r \mapsto \phi \circ \iota(r)$ is then an isomorphism of $R$-modules, and by Proposition 2(c), so is

$$
\operatorname{Hom}_{R}(M, N) \rightarrow \operatorname{Hom}_{R}\left(M \otimes_{R} A, N \otimes_{R} B\right), \quad g \mapsto g \otimes \phi .
$$

Since $f$ maps to an isomorphism $f \otimes \phi$ on the right hand side, it must itself be an isomorphism of modules. Finally, as $r \in R$ is a totally positive unit, $(N, r \cdot k)$ and $\left(B, \jmath, r^{-1} \cdot \mu\right)$ are objects of $\operatorname{Herm}_{1}(R)$ and $\mathcal{M}_{\Phi}^{1}(S)$. The relation (3.6) now shows that $f$ and $\phi$ are structure-preserving, hence morphisms in $\operatorname{Herm}_{n}(R)$ and $\mathcal{M}_{\Phi}^{1}(S)$ as claimed.

We will show that a given morphism can always be written as a composition of a pure tensor with a morphism of a particular type, which we now construct.

Let $(\mathfrak{a}, \alpha) \in \operatorname{Herm}_{1}(R),(B, \jmath, \mu) \in \mathcal{M}_{\Phi}^{1}(S)$, and suppose $\left(\mathfrak{a}^{\prime}, \alpha^{\prime}\right) \in \operatorname{Herm}_{1}(R)$ is an inverse of $(\mathfrak{a}, \alpha)$, meaning there exists an isomorphism

$$
\kappa:(\mathfrak{a}, \alpha) \otimes_{R}\left(\mathfrak{a}^{\prime}, \alpha^{\prime}\right) \stackrel{\sim}{\longrightarrow}(R, \mathbb{1}) .
$$

Let $\left(N^{\prime}, k^{\prime}\right)=(N, k) \otimes_{R}(\mathfrak{a}, \alpha)$ and $\left(B^{\prime}, \lambda^{\prime}, \mu^{\prime}\right)=\left(\mathfrak{a}^{\prime}, \alpha^{\prime}\right) \otimes(B, \lambda, \mu)$. Then we have an isomorphism

$$
\omega_{\kappa}:\left(N^{\prime}, k^{\prime}\right) \otimes\left(B^{\prime}, \jmath^{\prime}, \mu^{\prime}\right) \rightarrow(N, k) \otimes(B, \jmath, \mu),
$$

given on $T$-valued points by

$$
\left(\omega_{\kappa}\right)_{T}:\left(N \otimes_{R} \mathfrak{a}\right) \otimes_{R}\left(\mathfrak{a}^{\prime} \otimes_{R} B(T)\right) \rightarrow N \otimes_{R} B(T), \quad(n \otimes x) \otimes(y \otimes t) \mapsto n \otimes \kappa(x \otimes y) \cdot t .
$$

The map $\omega_{\kappa}$ is evidently an $R$-linear isomorphism of abelian schemes. In fact, it is also an isomorphism of triples. Verifying this amounts to showing the dual of an associator map is the expected associator of the duals. This can be checked using the explicit formula in Proposition 5 ,

The morphism $\omega_{\kappa}$ above is in general not a pure tensor. Indeed, assume $\omega_{\kappa}=f \otimes \phi$. By Lemma 39, $\phi: B^{\prime}=\mathfrak{a}^{\prime} \otimes_{R} B \rightarrow B$ must be an $R$-linear isomorphism, which implies $\mathfrak{a}^{\prime} \simeq R$, hence also $\mathfrak{a} \simeq R$. Then if $\mathfrak{a}$ is not a principal ideal $\omega_{k}$ is not a pure tensor.

The following theorem gives an explicit description of all the morphisms between objects produced by Serre's construction in $\mathcal{M}_{\Phi}^{n}$.

Theorem 40. Let $(A, \iota, \lambda),(B, \jmath, \mu) \in \mathcal{M}_{\Phi}^{1}(S)$ and $(M, h),(N, k) \in \operatorname{Herm}_{n}(R)$, and let

$$
\Psi:(M, h) \otimes(A, \iota, \lambda) \stackrel{\sim}{\longrightarrow}(N, k) \otimes(B, \jmath, \mu)
$$

be a morphism in $\mathcal{M}_{\Phi}^{n}$. Then $\Psi=\omega \circ(f \otimes \phi)$ for some $\omega$ as in (3.8), and

$$
f:(M, h) \stackrel{\sim}{\longrightarrow}(N, k) \otimes(\mathfrak{a}, \alpha), \quad \phi:(A, \iota, \lambda) \stackrel{\sim}{\longrightarrow}\left(\mathfrak{a}^{\prime} \otimes_{R} \alpha^{\prime}\right) \otimes(B, \jmath, \mu),
$$

morphisms in $\operatorname{Herm}_{n}(R)$ and $\mathcal{M}_{\Phi}^{1}$ respectively. Here, $\mathfrak{a}=\operatorname{Hom}_{R}(A, B), \mathfrak{a}^{\prime}=\operatorname{Hom}_{R}(B, A)$, and $\alpha$, $\alpha^{\prime}$ are the hermitian forms in Proposition $38(c)$, up to a totally positive unit $r \in R^{\times}$. The map $\omega$ is given on $T$-valued points by

$\omega_{T}:\left(N \otimes_{R} \mathfrak{a}\right) \otimes\left(\mathfrak{a}^{\prime} \otimes_{R} B(T)\right) \stackrel{\sim}{\longrightarrow} N \otimes_{R} B(T), \quad(n \otimes f) \otimes(g \otimes t) \mapsto n \otimes(g \circ f \circ t), \quad T \in \mathrm{Sch}_{/ S}$.

Proof. The map $\omega$ corresponds to $\omega_{k}$ in (3.8) where $\kappa$ is the isomorphism in Proposition 38(b). We put

and $\Psi_{0}=\omega^{-1} \circ \Psi$, so that

$$
\left(B^{\prime}, \jmath^{\prime}, \mu^{\prime}\right)=\left(\mathfrak{a}^{\prime}, \alpha^{\prime}\right) \otimes(B, \jmath, \mu), \quad\left(N^{\prime}, k^{\prime}\right)=(N, k) \otimes(\mathfrak{a}, \alpha),
$$

$$
\Psi_{0}:(M, h) \otimes(A, \iota, \lambda) \stackrel{\sim}{\longrightarrow}\left(N^{\prime}, k^{\prime}\right) \otimes\left(B^{\prime}, \jmath^{\prime}, \mu^{\prime}\right) .
$$

By Proposition [38(a) we have $B^{\prime} \simeq A$. Then $\operatorname{Hom}_{R}\left(A, B^{\prime}\right) \simeq R$, so by Proposition 2(c) every element of $\operatorname{Hom}_{R}\left(M \otimes_{R} A, N^{\prime} \otimes_{R} B^{\prime}\right)$ is a pure tensor, including $\Psi_{0}$. Hence by Lemma 39 there exists a totally positive unit $r \in R^{\times}$and isomorphisms

$$
f:(M, h) \stackrel{\sim}{\longrightarrow}\left(N^{\prime}, r \cdot k^{\prime}\right), \quad \phi:(A, \iota, \lambda) \stackrel{\sim}{\longrightarrow}\left(B^{\prime}, \jmath^{\prime}, r^{-1} \cdot \mu^{\prime}\right),
$$


in $\operatorname{Herm}_{1}(R)$ and $\mathcal{M}_{\Phi}^{1}(S)$, such that $\Psi_{0}=f \otimes \phi$. In particular, $\Psi=\omega \circ(f \otimes \phi)$.

We note that the map $\omega$ from the theorem has the same form as $\omega_{a, X, Y}$ of (2.3) from $\S 2$. Indeed, with notation as in the theorem, put $X=(N, k), Y=(B, \jmath, \mu)$, and $a=(\mathfrak{a}, \alpha)$. The pair $\left(\mathfrak{a}^{\prime}, \alpha^{\prime}\right)$ can be taken as $a^{-1}$, with $I_{a}: a \square a^{-1} \stackrel{\sim}{\longrightarrow} e$ given by $\kappa:(f \otimes g) \mapsto \iota^{-1}(g \circ f)$. Then $\omega_{a, X, Y}: X a \otimes a^{-1} Y \stackrel{\sim}{\longrightarrow} X \otimes Y$ of $(2.3)$ is mapped to $\omega$ of Theorem 40 by the Serre construction functor

$$
\begin{gathered}
\Sigma_{S}: \operatorname{Herm}_{n}(R) \otimes_{\mathrm{Herm}_{1}(R)} \mathcal{M}_{\Phi}^{1}(S) \longrightarrow \mathcal{M}_{\Phi}^{n}(S), \\
(M, h) \otimes(A, \iota, \lambda) \mapsto(M, h) \otimes(A, \iota, \lambda) .
\end{gathered}
$$

We now prove Proposition 36, claimed at the start of this section, that $\Sigma_{S}$ is fully faithful.

Proof of Proposition [36: Surjectivity of $\Sigma_{S}$ on morphisms follows from Theorem 40, since any morphism $\Psi$ in $\mathcal{M}_{\Phi}^{n}(S)$ has the form $\omega \circ(f \otimes \phi)$, and $\omega$ is in the image of $\Sigma_{S}$ as noted above. We show injectivity by comparing Theorem 40 with Proposition 24.

Given a morphism

$$
\tau:(M, h) \otimes(A, \iota, \lambda) \rightarrow(N, k) \otimes(B, \jmath, \mu)
$$

in the domain of $\Sigma_{S}$, by Proposition 24 we can write $\tau=\omega_{0} \circ(f \otimes \phi)$, where $\omega_{0}=\omega_{(N, k),(B, \jmath, \mu),(\mathfrak{a}, \alpha)}$ depends on $(\mathfrak{a}, \alpha),\left(\mathfrak{a}^{\prime}, \alpha^{\prime}\right) \in \operatorname{Herm}_{1}(R)$, and an isomorphism $I_{(\mathfrak{a}, \alpha)}:(\mathfrak{a}, \alpha) \otimes_{R}\left(\mathfrak{a}^{\prime}, \alpha^{\prime}\right) \rightarrow(R, \mathbb{1})$. The map $f \otimes \phi$ is of the form

$$
f \otimes \phi:(M, h) \otimes(A, \iota, \lambda) \rightarrow\left(N^{\prime}, k^{\prime}\right) \otimes\left(B^{\prime}, \jmath^{\prime}, \mu^{\prime}\right),
$$

with $\left(B^{\prime}, \jmath^{\prime}, \mu^{\prime}\right)=\left(\mathfrak{a}^{\prime}, \alpha^{\prime}\right) \otimes(B, \jmath, \mu)$ and $\left(N^{\prime}, k^{\prime}\right)=(N, k) \otimes_{R}(\mathfrak{a}, \alpha)$. We can replace any of the objects $A, B, \mathfrak{a}$ and $\mathfrak{a}^{\prime}$ by isomorphic ones since that does not alter the general form $\omega_{0} \circ(f \otimes \phi)$, as long as we also supply $I_{(\mathfrak{a}, \alpha)}$.

Since $\phi: A \rightarrow B^{\prime}$ is an isomorphism, we have $\mathfrak{a} \simeq \operatorname{Hom}_{R}(A, B)$ and $\mathfrak{a}^{\prime} \simeq \operatorname{Hom}_{R}(B, A)$, equipped with some $\alpha, \alpha^{\prime}$. The hermitian forms on $\mathfrak{a}, \mathfrak{a}^{\prime}$ given by Proposition 38(c) are $r \alpha$ and $r^{-1} \alpha^{\prime}$ for some totally positive $r \in R^{\times}$. Regardless of the value of $r$, the map in Proposition 38(b) can be taken as $I_{(\mathfrak{a}, \alpha)}$. With this choice of $\mathfrak{a}, \mathfrak{a}^{\prime}$ and $I_{(\mathfrak{a}, \alpha)}$, we have $\Sigma_{S}\left(\omega_{0}\right)=\omega$, where $\omega$ is as in Theorem 40.

Now let $\tau_{0}$ be another morphism such that $\Sigma_{S}\left(\tau_{0}\right)=\Sigma_{S}(\tau)$. We must show $\tau_{0}=\tau$. By the same argument as above, $\tau_{0}$ can be written as $\omega_{0} \circ\left(f_{0} \otimes \phi_{0}\right)$ with the same $\omega_{0}$. Then

$$
f \otimes \phi=\omega^{-1} \circ \Sigma_{S}(\tau)=\omega^{-1} \circ \Sigma_{S}\left(\tau_{0}\right)=f_{0} \otimes \phi_{0} .
$$

It remains to show this implies $f \otimes \phi=f_{0} \otimes \phi_{0}$.

Since $\phi_{0}: A \rightarrow B^{\prime}$ and $\phi: A \rightarrow B^{\prime}$ are $R$-linear isomorphisms, we have $\phi_{0}=r \cdot \phi$ for some $r \in R$. From $f \otimes \phi=f_{0} \otimes \phi_{0}$, we get $f=r \cdot f_{0}$. Now $\iota(r)=\phi_{0} \circ \phi^{-1}$ is an automorphism of $(A, \iota, \lambda)$, so $\lambda=\iota(r)^{\vee} \circ \lambda \circ \iota(r)=\lambda \circ \iota\left(r^{\sigma} r\right)$, and $r^{\sigma} r=1$. It follows that $\mu_{r}:(R, \mathbb{1}) \rightarrow(R, \mathbb{1}), x \mapsto r x$ is an automorphism of $(R, \mathbb{1})$. Now, we have a diagram

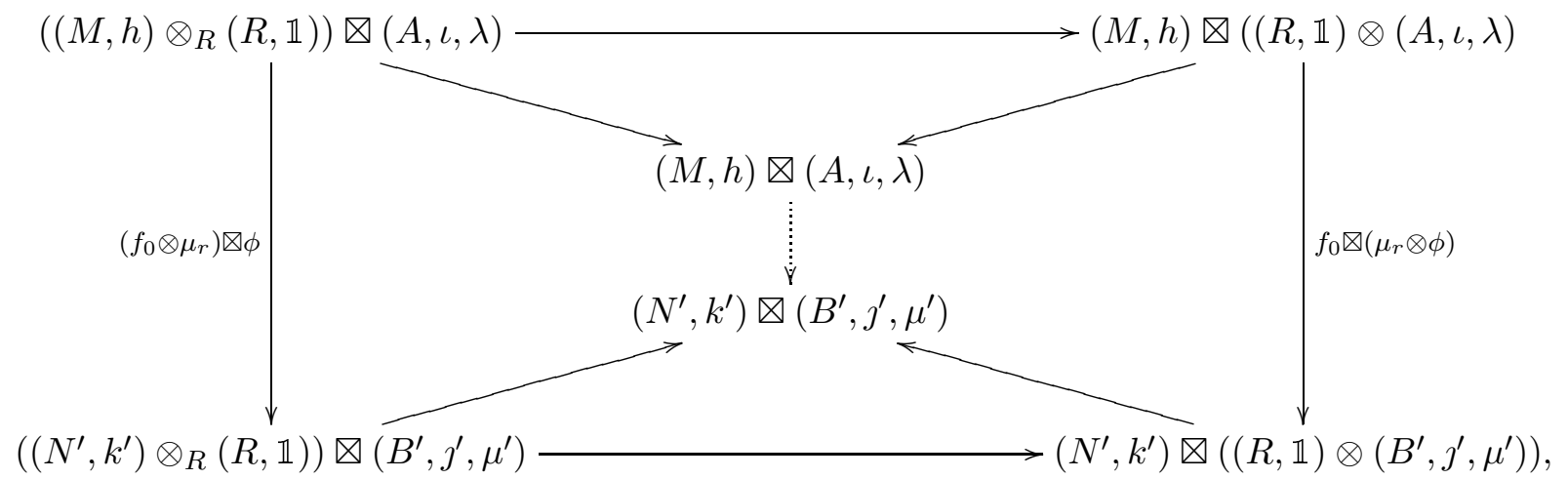


where the oblique arrows are canonical isomorphisms. The square and the two triangles commute by the axioms of the categorical tensor product. The right trapezoid commutes if and only if the dotted arrow is $f_{0} \otimes(r \cdot \phi)=f_{0} \otimes \phi_{0}$, and the left trapezoid commutes if and only if it is $\left(r \cdot f_{0}\right) \otimes \phi=f \otimes \phi$. The commutativity of each trapezoid implies the other, therefore $f \otimes \phi=f_{0} \otimes \phi_{0}$, and $\tau=\tau_{0}$.

3.3. Serre construction of $\mathcal{M}_{\Phi}^{n}$ : the objects. In this section we look for triples in $\mathcal{M}_{\Phi}^{n}(S)$ that come from the Serre construction. When $S=\operatorname{Spec} \mathbb{C}$, every object is Serre constructible using an equivalence between $\mathcal{M}_{\Phi}^{n}(\mathbb{C})$ and a category of linear-algebraic data. For general $S$, every object of $\mathcal{M}_{\Phi}^{n}(S)$ is étale locally on the base Serre constructible. This is done by using Theorem 35 to reduce to the complex case.

The following lemma simplifies the task of detecting Serre constructible triples.

Lemma 41. Suppose $(B, \jmath, \mu)$ and $(A, \iota, \lambda)$ are objects in $\mathcal{M}_{\Phi}^{n}(S)$ and $\mathcal{M}_{\Phi}^{1}(S)$ respectively, $M$ is a projective finitely presented $\mathcal{O}_{K}$-module of rank $n$, and $\Psi: M \otimes_{\mathcal{O}_{K}} A \rightarrow B$ is an $\mathcal{O}_{K}$-linear isomorphism of abelian schemes. Then there exists a unique $h: M \rightarrow M^{\vee}$ such that $(M, h) \in$ $\operatorname{Herm}_{n}\left(\mathcal{O}_{K}\right)$, and $\Psi:(M, h) \otimes(A, \iota, \lambda) \rightarrow(B, \jmath, \mu)$ is an isomorphism of triples.

Proof. The map $\lambda_{M}=\Psi^{\vee} \circ \mu \circ \Psi$ is an $\mathcal{O}_{K}$-linear principal polarization on $M \otimes_{\mathcal{O}_{K}} A$. As $\lambda$ is a basis for $\operatorname{Hom}_{\mathcal{O}_{K}}\left(A, A^{\vee}\right)$, by Proposition 2 (c) we have $\lambda_{M}=h \otimes \lambda$ for a unique $\mathcal{O}_{K}$-linear map $h: M \rightarrow M^{\vee}$. By Proposition [18, $(M, h) \in \operatorname{Herm}_{n}\left(\mathcal{O}_{K}\right)$. Then $(M, h) \otimes(A, \iota, \lambda)$ is an object of $\mathcal{M}_{\Phi}^{n}(S)$, and $\Psi$ is an isomorphism of triples.

We first consider the case $S=\operatorname{Spec} \mathbb{C}$. For any field embedding $\phi: K \rightarrow \mathbb{C}$, let $\mathbb{C}^{(\phi)}$ denote $\mathbb{C}$ as a $K \otimes \mathbb{C}$-algebra, with structure homomorphism $K \otimes \mathbb{C} \rightarrow \mathbb{C}^{(\phi)}, a \otimes z \rightarrow \phi(a) z$. Then $\mathbb{C}^{\Phi}=\bigoplus_{\phi \in \Phi} \mathbb{C}^{(\phi)}$ is also a $K \otimes \mathbb{C}$-algebra. If $V$ is any $K$-vector space, there's a $K$-linear isomorphism

$$
V \otimes \mathbb{R} \cong V \otimes_{K}(K \otimes \mathbb{R}) \simeq V \otimes_{K} \mathbb{C}^{\Phi} \cong \bigoplus_{\phi \in \Phi} V \otimes_{K} \mathbb{C}^{(\phi)} .
$$

Lemma 42. Let $(A, \iota, \lambda) \in \mathcal{M}_{\Phi}^{n}(\mathbb{C})$ and $V=H_{1}(A, \mathbb{Q})$. If $V \otimes \mathbb{R}$ is equipped with the $\mathbb{C}$-vector space structure induced by $A$ as a complex variety, the isomorphism of (3.9) is $K \otimes \mathbb{C}$-linear.

Proof. From the Hodge filtration $\operatorname{Fil}^{1} H_{1}(A, \mathbb{C}) \subset H_{1}(A, \mathbb{C})$, we have

$$
H_{1}(A, \mathbb{R}) \cong H_{1}(A, \mathbb{C}) / \operatorname{Fil}^{1} H_{1}(A, \mathbb{C}) \cong \operatorname{Lie}(A) .
$$

By Proposition [32, Fil $^{1} H_{1}(A, \mathbb{C})=J_{\Phi} H_{1}(A, \mathbb{C})$. Then we have $K \otimes \mathbb{C}$-linear isomorphisms

$$
H_{1}(A, \mathbb{C}) / J_{\Phi} H_{1}(A, \mathbb{C}) \cong H_{1}(A, \mathbb{C}) \otimes_{K \otimes \mathbb{C}}\left(K \otimes \mathbb{C} / J_{\Phi}(K \otimes \mathbb{C})\right) \cong H_{1}(A, \mathbb{C}) \otimes_{K \otimes \mathbb{C}} \mathbb{C}^{\Phi} .
$$

The resulting $K \otimes \mathbb{C}$-linear map $H_{1}(A, \mathbb{R}) \rightarrow H_{1}(A, \mathbb{C}) \otimes_{K \otimes \mathbb{C}} \mathbb{C}^{\Phi}$ is evidently the same as (3.9).

By the general theory of complex abelian varieties, the isomorphism class of a triple $(A, \iota, \lambda)$ over $\mathbb{C}$ is uniquely determined by the $\mathcal{O}_{K}$-module $H=H_{1}(A, \mathbb{Z})$, the Riemann form $E: H \times H \rightarrow \mathbb{Z}$, and the complex structure on $H \otimes \mathbb{R} \cong \operatorname{Lie}(A)$. For $(A, \iota, \lambda) \in \mathcal{M}_{\Phi}^{n}(\mathbb{C})$, the lemma shows the complex structure is determined by the other data.

Let $H$ be a projective finitely presented $\mathcal{O}_{K}$-module. Suppose $E: H \times H \rightarrow \mathbb{Z}$ is alternating, with

$$
E(\alpha x, y)=E\left(x, \alpha^{\sigma} y\right), \quad \alpha \in \mathcal{O}_{K}, x, y \in H .
$$

Then $E=\operatorname{Tr}_{K / \mathbb{Q}} F$ for a unique $\delta_{K}^{-1}$-valued skew-hermitian form $F: H \times H \rightarrow \delta_{K}^{-1}$, where $\delta_{K}^{-1}$ is the inverse different of $K / \mathbb{Q}$. Let $\left\{\alpha_{j}\right\}$ be a $\mathbb{Z}$-basis for $\mathcal{O}_{K}$, and $\left\{\beta_{j}\right\}$ the trace-dual basis for $\delta_{K}^{-1}$. Then

$$
F(x, y)=\sum_{j} E\left(x, \alpha_{j} y\right) \beta_{j}
$$


For any $x \in H, \zeta=F(x, x)$ satisfies $\zeta=-\zeta^{\sigma}$. Since $K$ is a CM field, then also $\phi(\zeta)=-\phi(\zeta)^{\sigma}$ for all $\phi \in \operatorname{Hom}(K, \mathbb{C})$. Hence if $\zeta \neq 0$, there exists a unique CM-type $\Psi \subset \operatorname{Hom}(K, \mathbb{C})$ such that $\operatorname{Im}(\psi(\zeta))<0$ for all $\psi \in \Psi$.

Definition 43. Suppose $\mathfrak{d} \subset K$ is a fractional ideal satisfying $\mathfrak{d}^{\sigma}=\mathfrak{d}$. A $\mathfrak{d}$-valued skew hermitian form $F: H \times H \rightarrow \mathfrak{d}$ is called negative-definite along $\Phi$, if $\operatorname{Im}(\phi(F(x, x)))<0$ for all non-zero $x \in H, \phi \in \Phi$.

Now put $U=H \otimes \mathbb{R}$. Then $U \simeq H_{\mathbb{Q}} \otimes_{K} \mathbb{C}^{\Phi}$ via (3.9) is a $\mathbb{C}$-vector space.

Lemma 44. $E$ is a Riemann form for $U / H$ if and only if $F$ is negative-definite along $\Phi$.

Proof. Let $E_{\mathbb{R}}$ denote the $\mathbb{R}$-linear extension of $E$ to $U \times U$. Let $U^{(\phi)}$ be the subspace of $U=H \otimes \mathbb{R}$ corresponding to $H_{\mathbb{Q}} \otimes_{K} \mathbb{C}^{(\phi)} \subset H_{\mathbb{Q}} \otimes_{K} \mathbb{C}^{\Phi}$ via (3.9). We have an orthogonal decomposition $E_{\mathbb{R}}=\bigoplus_{\phi \in \Phi} E^{(\phi)}$, where $E^{(\phi)}=\left.E_{\mathbb{R}}\right|_{U^{(\phi)} \times U^{(\phi)}}$. Let $F_{\mathbb{R}}=\bigoplus_{\phi \in \Phi} F^{(\phi)}$ be the analogous $\mathbb{R}$-linear extension of $F$. Each $F^{(\phi)}$ is the $\mathbb{C}$-linear extension of $F$ to $U^{(\phi)} \times U^{(\phi)}$, induced by $\phi$, with values in $\mathbb{C}^{(\phi)}$.

For each $\phi \in \Phi$, using (3.11) we have

$$
F^{(\phi)}(x, y)=\left(\sum_{j} \operatorname{Re}\left(\phi\left(\alpha_{j}\right)\right) \phi\left(\beta_{j}\right)\right) E^{(\phi)}(x, y)+\left(\sum_{j} \operatorname{Im}\left(\phi\left(\alpha_{j}\right)\right) \phi\left(\beta_{j}\right)\right) E^{(\phi)}(x, i y),
$$

where $\left\{\alpha_{j}\right\},\left\{\beta_{j}\right\}$ are trace-dual bases for $\mathcal{O}_{K}$ and $\delta_{K}^{-1}$, respectively. As $K$ is CM, we have

$$
\sum_{j} \alpha_{j} \beta_{j}=1, \quad \sum_{j} \alpha_{j}^{\sigma} \beta_{j}=0
$$

from which

$$
\sum_{j} \operatorname{Re}\left(\phi\left(\alpha_{j}\right)\right) \phi\left(\beta_{j}\right)=\frac{1}{2}, \quad \sum_{j} \operatorname{Im}\left(\phi\left(\alpha_{j}\right)\right) \phi\left(\beta_{j}\right)=\frac{i}{2}
$$

It follows that

$$
F^{(\phi)}(x, y)=\frac{1}{2} E^{(\phi)}(x, y)+\frac{i}{2} E^{(\phi)}(x, i y), \quad \forall x, y \in U^{(\phi)},
$$

and in particular $F^{(\phi)}(x, x)=-\frac{i}{2} E^{(\phi)}(i x, x)$. Then by the orthogonal decompositions of $F$ and $E$, $F$ is negative-definite along $\Phi$ if and only if $E(i x, y)$ is positive-definite.

Now put $H^{*}=\operatorname{Hom}_{\mathcal{O}_{K}}\left(H, \delta_{K}^{-1}\right) \cong H^{\vee} \otimes_{\mathcal{O}_{k}} \delta_{K}^{-1}$. A $\delta_{K}^{-1}$-valued sesquilinear form $F$ corresponds to an $\mathcal{O}_{K}$-linear map $f: H \rightarrow H^{*}$ via $f(x)(y)=F(x, y)$. We identify $f$ with $F$ in this way, and speak of skew-hermitian forms $f: H \rightarrow H^{*}$. We have $\left(H^{*}\right)^{*} \cong H$ canonically, so that $H \mapsto H^{*}$ is a duality on the category of projective finitely presented $\mathcal{O}_{K}$-modules. Then $f$ is skew-hermitian if and only if $f^{*}=-f$. As usual, $f$ is non-degenerate if it is an isomorphism, and that's the case if and only if $E=\operatorname{Tr}_{K / \mathbb{Q}} F$ is non-degenerate.

More generally, suppose $\mathfrak{d}$ is a fractional ideal of $K$ such that $\mathfrak{d}^{\sigma}=\mathfrak{d}$. For $\epsilon= \pm 1$, a $\mathfrak{d}$-valued sesquilinear form $G: H \times H \rightarrow \mathfrak{d}$ is called $\epsilon$-hermitian if $H(x, y)^{\sigma}=\epsilon H(y, x)$. Such $G$ correspond to $\mathcal{O}_{K}$-linear maps $g: H \rightarrow \operatorname{Hom}_{\mathcal{O}_{K}}(H, \mathfrak{d}) \cong H^{\vee} \otimes_{\mathcal{O}_{K}} \mathfrak{d}$. If $(H, g)$ is $\mathfrak{d}$-valued $\epsilon$-hermitian, and $\left(H^{\prime}, g^{\prime}\right)$ is $\mathfrak{d}^{\prime}$-valued $\epsilon^{\prime}$-hermitian, then $(H, g) \otimes\left(H^{\prime}, g^{\prime}\right)=\left(H \otimes \mathcal{O}_{K} H^{\prime}, g \otimes g^{\prime}\right)$ is $\mathfrak{d} \mathfrak{d}^{\prime}$-valued $\epsilon \epsilon^{\prime}$-hermitian. In particular, $\left(H \otimes H^{\prime}, g \otimes g^{\prime}\right)$ is skew-hermitian if one of $(H, g)$ and $\left(H^{\prime}, g^{\prime}\right)$ is hermitian, and the other skew-hermitian. If $(H, g)$ and $\left(H^{\prime}, g^{\prime}\right)$ are either both skew-hermitian or both hermitian, $\left(H \otimes H^{\prime}, g \otimes g^{\prime}\right)$ is hermitian. If $(H, g)$ is $\mathfrak{d}$-valued skew-hermitian and negative-definite along $\Phi$ (Definition 43), and $\left(H^{\prime}, g^{\prime}\right)$ is $\mathfrak{d}^{-1}$-valued skew-hermitian and negative-definite along $\Phi \sigma$, then $(H, g) \otimes\left(H^{\prime}, g^{\prime}\right)$ is $\mathcal{O}_{K}$-hermitian and positive-definite. 
Let $\operatorname{Skew}_{\Phi}^{n}\left(\mathcal{O}_{K}\right)$ denote the category of pairs $(H, f)$, where $H$ is a projective finitely presented $\mathcal{O}_{K}$-module of rank $n$, and $f: H \rightarrow H^{*}$ is non-degenerate $\delta_{K}^{-1}$-valued skew-hermitian, and negativedefinite along $\Phi$. The morphisms are isomorphisms of $\mathcal{O}_{K}$-modules which preserve the forms. The 2-group $\operatorname{Herm}_{1}\left(\mathcal{O}_{K}\right)$ acts on $\operatorname{Skew}_{\Phi}^{n}\left(\mathcal{O}_{K}\right)$ via ordinary tensor product. In other words, if $(H, f) \in \operatorname{Skew}_{\Phi}^{n}\left(\mathcal{O}_{K}\right)$ and $(\mathfrak{a}, \alpha) \in \operatorname{Herm}_{1}\left(\mathcal{O}_{K}\right)$, then $\left(H \otimes_{\mathcal{O}_{K}} \mathfrak{a}, f \otimes \alpha\right) \in \operatorname{Skew}_{\Phi}^{n}\left(\mathcal{O}_{K}\right)$.

For each $(A, \iota, \lambda) \in \mathcal{M}_{\Phi}^{n}(\mathbb{C})$, Let $\Theta(A, \iota, \lambda)=(H, f)$, where $H=H_{1}(A, \mathbb{Z})$ and $f$ is

$$
H_{1}(A, \mathbb{Z}) \stackrel{H_{1}(\lambda)}{\longrightarrow} H_{1}\left(A^{\vee}, \mathbb{Z}\right) \cong H_{1}(A, \mathbb{Z})^{*} .
$$

Then we have a functor

$$
\Theta=\Theta_{n}: \mathcal{M}_{\Phi}^{n}(\mathbb{C}) \longrightarrow \operatorname{Skew}_{\Phi}^{n}\left(\mathcal{O}_{K}\right)
$$

Proposition 45. $\Theta$ is an equivalence of categories. It is $\operatorname{Herm}_{1}\left(\mathcal{O}_{K}\right)$-equivariant up to canonical isomorphism.

Proof. This follows from the basic theory of complex abelian varieties. A quasi-inverse to $\Theta$ is given by

$$
\Xi: \operatorname{Skew}_{\Phi}^{n}\left(\mathcal{O}_{K}\right) \longrightarrow \mathcal{M}_{\Phi}^{n}(\mathbb{C}), \quad \Xi(H, f)=(H \otimes \mathbb{R}) / H
$$

where $(H \otimes \mathbb{R}) / H$ is a complex torus via (3.9), polarized by $E(x, y)=\operatorname{Tr}_{K / \mathbb{Q}}(f(x)(y))$. The isomorphism of functors $\mathbb{1}_{\mathcal{M}_{\Phi}^{n}(\mathbb{C})} \cong \Xi \circ \Theta$ corresponds to the canonical uniformization of a complex abelian variety. The other direction $\mathbb{1}_{\text {Skew }_{\Phi}^{n}\left(\mathcal{O}_{K}\right)} \simeq \Theta \circ \Xi$ is induced by $H \cong H_{1}(H \otimes \mathbb{R} / H)$. It's straight-forward to check these are isomorphisms.

Checking $\Xi$ is equivariant implies the same for $\Theta$. For $A=(H \otimes \mathbb{R}) / H$, that corresponds to $\mathfrak{a} \otimes_{\mathcal{O}_{K}} A \cong\left(H_{\mathfrak{a}} \otimes \mathbb{R}\right) / H_{\mathfrak{a}}$ with $H_{\mathfrak{a}}=\mathfrak{a} \otimes_{\mathcal{O}_{K}} H$. That the isomorphism preserves polarizations can be checked using the Appell-Humbert data of the corresponding Poincaré bundles. Ultimately one must check that canonical duality isomorphisms are compatible with associators. This follows from the explicit form of the duality isomorphism in Theorem 5 .

For the domain of the functor

$$
\Sigma_{\mathbb{C}}: \operatorname{Herm}_{n}\left(\mathcal{O}_{K}\right) \otimes_{\operatorname{Herm}_{1}\left(\mathcal{O}_{K}\right)} \mathcal{M}_{\Phi}^{1}(\mathbb{C}) \rightarrow \mathcal{M}_{\Phi}^{n}(\mathbb{C})
$$

to be non-empty, $\mathcal{M}_{\Phi}^{1}(\mathbb{C})$ must contain objects. The following theorem shows this is almost always the case.

Theorem 46. $\mathcal{M}_{\Phi}^{1}(\mathbb{C}) \neq \emptyset$ for all $C M$ types $\Phi$, unless $K / F$ is unramified at every finite place. In that case, $\mathcal{M}_{\Phi}^{1}(\mathbb{C}) \neq \emptyset$ for exactly half the types.

Proof. Assume that for some CM type $\Phi_{0}, \mathcal{M}_{\Phi_{0}}^{1}(\mathbb{C})$ is non-empty. As explained in [26, IV] that means we can find a fractional ideal $\mathfrak{a} \subset K$ and an element $\zeta \in K$ such that

$$
\mathfrak{a a}^{\sigma} \delta_{K}=(\zeta), \quad \zeta^{\sigma}=-\zeta, \quad \operatorname{Im} \phi(\zeta)<0 \quad \forall \phi \in \Phi_{0},
$$

where $\delta_{K}$ is the different ideal of $K$. If $\mathcal{M}_{\Phi}^{1}(\mathbb{C}) \neq \emptyset$ for some other CM type $\Phi$, we get another pair $(\mathfrak{b}, \xi)$ with the corresponding properties. If we put $\mathfrak{c}=\mathfrak{b a}^{-1}$ and $r=\xi \zeta^{-1}$, then $r \in F, \mathfrak{c c}^{\sigma}=(r)$, and for any $\psi: F \hookrightarrow \overline{\mathbb{Q}}$, we have $\psi(r)>0$ if and only if $\Phi \cap \Phi_{0}$ contains an element of $\operatorname{Hom}(K, \overline{\mathbb{Q}})$ extending $\psi$.

Let $N_{0}(K)$ denote the group of pairs $(\mathfrak{c}, r)$, where $\mathfrak{c}$ is a non-zero fractional ideal of $K, r \in F^{\times}$, and $\mathfrak{c c}^{\sigma}=(r)$. Given $(\mathfrak{c}, r) \in N_{0}(K)$, we may put $\mathfrak{b}=\mathfrak{a c}$ and $\xi=r \zeta$. Then $\mathfrak{b}^{\sigma} \delta_{K}=(\xi)$, $\xi^{\sigma}=-\xi$, and there exists a unique CM type $\Phi$ such that $(\mathfrak{b}, \xi)$ defines an object of $\mathcal{M}_{\Phi}^{1}(\mathbb{C})$. Then $(\mathfrak{c}, r) \cdot \Phi_{0}=\Phi$ defines a transitive action of $N_{0}(K)$ on the set of CM types $\Phi$ for which $\mathcal{M}_{\Phi}^{1}(\mathbb{C})$ is non-empty. Let $N_{0}^{+}(K)$ denote the kernel of this action. It coincides with the stabilizer of any $\Phi_{0}$, and consists of all pairs $(\mathfrak{c}, r)$ such that $\mathfrak{c c}^{\sigma}=(r)$ and $r \in F^{\times}$is totally positive. Then the number of CM types $\Phi$ such that $\mathcal{M}_{\Phi}^{1}(\mathbb{C}) \neq \emptyset$ is equal to

$$
\left|N_{0}(K) / N_{0}^{+}(K)\right| \text {. }
$$


Let $U_{K}, I_{K}$, and $P_{K}$ denote the units of $\mathcal{O}_{K}$, the non-zero fractional ideals of $K$, and its subgroup of principal ideals, so that $C_{K}=I_{K} / P_{K}$ is the ideal class group. We also use the corresponding notation for $F$. Let $N_{K} \subset I_{K}$ consist of $\mathfrak{c}$ such that $\mathrm{Nm}_{K / F}(\mathfrak{c})=\mathfrak{c c}^{\sigma}$ is principal and generated by an element of $F$. We have a surjective map $N_{0}(K) \rightarrow N_{K},(\mathfrak{c}, r) \mapsto \mathfrak{c}$ and an exact sequence

$$
0 \rightarrow U_{F} \rightarrow N_{0}(K) \rightarrow N_{K} \rightarrow 0
$$

where $u \in U_{F}$ is identified with $\left(\mathcal{O}_{K}, u\right) \in N_{0}(K)$.

Let $P_{F}^{+} \subset P_{F}$ denote the subgroup of principal ideals that admit a totally positive generator, so that $C_{F}^{+}=I_{F} / P_{F}^{+}$is the narrow class group of $F$. We also have a subgroup $N_{K}^{+} \subset I_{K}$ consisting of $\mathfrak{c}$ such that $\mathrm{Nm}_{K / F}^{+}(\mathfrak{c})=\mathfrak{c c}^{\sigma}$ is in $P_{F}^{+}$. We get another exact sequence

$$
0 \rightarrow U_{F}^{+} \rightarrow N_{0}^{+}(K) \rightarrow N_{K}^{+} \rightarrow 0,
$$

where $U_{F}^{+} \subset U_{F}$ consists of totally positive units.

From the two exact sequences and the nine lemma we obtain another exact sequence

$$
0 \rightarrow U_{F} / U_{F}^{+} \rightarrow N_{0}(K) / N_{0}^{+}(K) \rightarrow N_{K} / N_{K}^{+} \rightarrow 0 .
$$

Note that $N_{K}$ and $N_{K}^{+}$both contain $P_{K}$, so that $N_{K} / N_{K}^{+}=\bar{N}_{K} / \bar{N}_{K}^{+}$, with $\bar{N}_{K}=N_{K} / P_{K}$ and $\bar{N}_{K}^{+}=N_{K}^{+} / P_{K}$ considered as subgroups of $C_{K}$. We then have an exact diagram

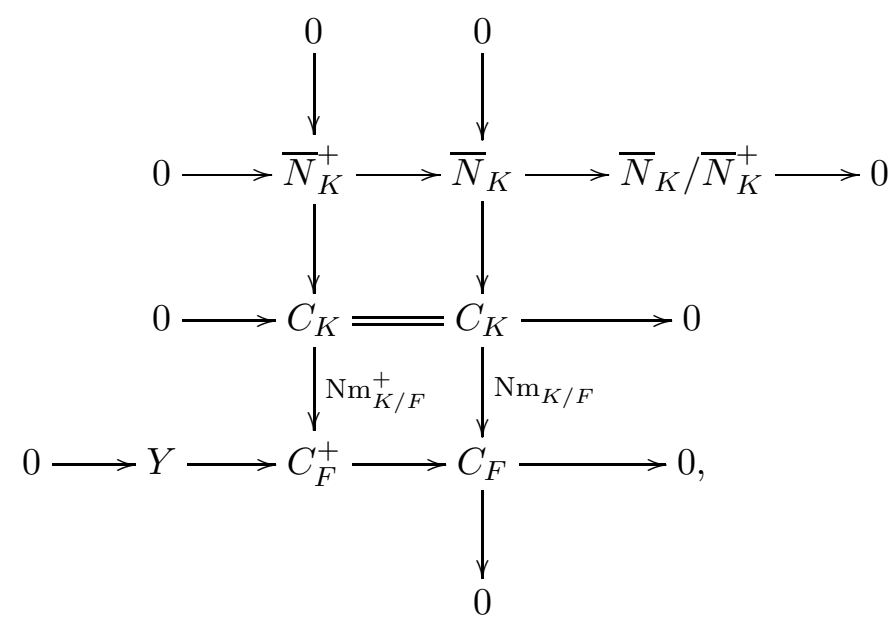

where $Y$ is kernel of $C_{F}^{+} \rightarrow C_{F}$. To see that the norm map $\operatorname{Nm}_{K / F}: C_{K} \rightarrow C_{F}$ is surjective, note that under the reciprocity isomorphism it corresponds to the restriction map $\operatorname{Gal}\left(H_{K} / K\right) \rightarrow$ $\operatorname{Gal}\left(H_{F} / F\right)$. Here $H_{K}$ and $H_{F}$ are the Hilbert class fields of $K$ and $F$, and $H_{F} \subset H_{K}$.

For the narrow Hilbert class fields $H_{K}^{+}$and $H_{F}^{+}$, we have $H_{K}^{+}=H_{K}$ since $K$ is totally imaginary, and so $H_{F}^{+} \subset H_{K}$. Again the map $\mathrm{Nm}_{K / F}^{+}: C_{K} \rightarrow C_{F}^{+}$corresponds to the restriction $\operatorname{Gal}\left(H_{K} / K\right) \rightarrow$ $\operatorname{Gal}\left(H_{F}^{+} / F\right)$. The latter is surjective if $K \cap H_{F}^{+}=F$, otherwise the image has index 2, with the quotient isomorphic to $\operatorname{Gal}(K / F)$. By a standard diagram chase we get an injection $\bar{N}_{K} / \bar{N}_{K}^{+} \rightarrow Y$, induced by $\mathrm{Nm}_{K / F}^{+}$, which is an isomorphism if $K / F$ is ramified at any finite prime, and an injection onto a subgroup of index 2 otherwise.

Now fix an ordering $\left(\psi_{1}, \cdots, \psi_{g}\right)$ of $\operatorname{Hom}(F, \overline{\mathbb{Q}})$, put $S=\{ \pm 1\}^{[F: \mathbb{Q}]}$, and consider the homomorphism $U_{F} \rightarrow S, u \mapsto s=\left(s_{1}, \cdots, s_{g}\right)$, where $s_{i}=\psi_{i}(u) /\left|\psi_{i}(u)\right|$. The kernel of this map is $U_{F}^{+}$, hence its image is isomorphic to $U_{F} / U_{F}^{+}$. The cokernel of this map is in fact isomorphic to $Y$, the kernel of $C_{F}^{+} \rightarrow C_{F}$, and also to $\operatorname{Gal}\left(H_{F}^{+} / H_{F}\right)$ [3, p. 47, Lemma 11.2]. Indeed, let $F^{+}$denote the totally positive elements of $F^{\times}$. By the (weak) approximation theorem the map $F^{\times} \rightarrow S$ extending $U_{F} \rightarrow S$ is surjective, so that $S \cong F^{\times} / F^{+}$. Then the cokernel of $U_{F} \rightarrow S$ is isomorphic 
to $\left(F^{\times} / F^{+}\right) /\left(U_{F} / U_{F}^{+}\right) \cong F^{\times} / U_{F} F^{+} \cong P_{F} / P_{F}^{+} \cong Y$. It follows that $|Y| \cdot\left|U_{F} / U_{F}^{+}\right|=|S|$, and so from $\bar{N}_{K} / \bar{N}_{K}^{+}=N_{K} / N_{K}^{+}$and (3.14) we obtain

$$
\left|N_{0}(K) / N_{0}^{+}(K)\right|= \begin{cases}2^{g-1} & \text { if } K / F \text { is unramified at all finite primes } \\ 2^{g} & \text { otherwise }\end{cases}
$$

Now note the surjectivity of $\mathrm{Nm}_{K / F}: C_{K} \rightarrow C_{F}$ implies our initial assumption that $\mathcal{M}_{\Phi_{0}}^{1}(\mathbb{C}) \neq \emptyset$ for some $\Phi_{0}$. Indeed, for an arbitrary totally imaginary $\zeta_{0} \in K^{\times}$, the fractional ideal $\zeta_{0} \delta_{K}^{-1}$ descends to a fractional ideal of $F$. Then there exists $\mathfrak{a} \in I_{K}$ such that $N_{K / F}(\mathfrak{a}) \zeta_{0}^{-1} \delta_{K}$ is principal, generated by some $r \in F$. In other words $\mathfrak{a a}^{\sigma} \delta_{K}=(\zeta)$, where $\zeta=r \zeta_{0}$ is totally imaginary. Letting $\Phi_{0}$ be the unique CM type such that $\operatorname{Im}(\phi(\zeta))<0$ for all $\phi \in \Phi_{0}$, we obtain a pair $(\mathfrak{a}, \zeta)$ corresponding to an object of $\mathcal{M}_{\Phi_{0}}^{1}(\mathbb{C})$.

Thus we have shown that if $K / F$ is ramified at any finite prime, the number of CM types $\Phi$ such that $\mathcal{M}_{\Phi}^{1}(\mathbb{C}) \neq \emptyset$ is $2^{g}$, which is the number of all CM types. If $K / F$ is unramified at every finite prime, then $\mathcal{M}_{\Phi}^{1}(\mathbb{C}) \neq \emptyset$ for $2^{g-1} \mathrm{CM}$ types, which is exactly half of them.

We note that if $\mathcal{M}_{\Phi}^{1}(\mathbb{C}) \neq \emptyset$ for some $\Phi$, there exists a number field $L_{0}$ such that $\mathcal{M}_{\Phi}^{1}\left(\operatorname{Spec} \mathcal{O}_{L_{0}}\right) \neq$ $\emptyset$. This follows the fact that $\mathrm{CM}$ abelian varieties have potential good reduction everywhere, using Néron models.

If $n$ is odd, we offer an explicit way to construct an object of $\mathcal{M}_{\Phi}^{1}(\mathbb{C})$ given one in $\mathcal{M}_{\Phi}^{n}(\mathbb{C})$, via Proposition 45 and the following.

Proposition 47. Assume that $n=2 m+1$, and $(H, f) \in \operatorname{Skew}_{\Phi}^{n}\left(\mathcal{O}_{K}\right)$. Then $(\mathfrak{a}, \alpha) \in \operatorname{Skew}_{\Phi}^{1}\left(\mathcal{O}_{K}\right)$, where

$$
\mathfrak{a}=\operatorname{det}(H) \otimes_{\mathcal{O}_{K}} \delta_{K}^{m}, \quad \alpha=(-1)^{m}(\operatorname{det}(f) \otimes \sigma) .
$$

Proof. Since $H$ has rank $n, \operatorname{det}(H)=\bigwedge_{\mathcal{O}_{K}}^{n} H$ is projective of rank one. Since $f$ is an isomorphism, so is $\operatorname{det}(f): \operatorname{det}(H) \rightarrow \operatorname{det}\left(H^{*}\right)$.

Letting $\mathfrak{a}=\operatorname{det}(H) \otimes_{\mathcal{O}_{K}} \delta_{K}^{m}$, we have canonical isomorphisms

$\operatorname{det}\left(H^{*}\right) \otimes_{\mathcal{O}_{K}} \delta_{K}^{m} \cong \operatorname{det}\left(H^{\vee} \otimes_{\mathcal{O}_{K}} \delta_{K}^{-1}\right) \otimes_{\mathcal{O}_{K}} \delta_{K}^{m} \cong \operatorname{det}(H)^{\vee} \otimes_{\mathcal{O}_{K}} \delta_{K}^{m-n} \cong\left(\operatorname{det}(H) \otimes_{\mathcal{O}_{K}} \delta_{K}^{m}\right)^{\vee} \otimes_{\mathcal{O}_{K}} \delta_{K}^{-1} \cong \mathfrak{a}^{*}$, where we have used $\operatorname{det}\left(H^{\vee} \otimes_{\mathcal{O}_{K}} \delta_{K}^{-1}\right) \cong \operatorname{det}\left(H^{\vee}\right) \otimes_{\mathcal{O}_{K}} \delta_{K}^{-n}$ and $\delta_{K}^{\vee} \cong \delta_{K}^{-1}$ in the middle, along with $m-n=-m-1$.

Using the above isomorphism, we obtain $\alpha: \mathfrak{a} \rightarrow \mathfrak{a}^{*}$, corresponding to

$$
(-1)^{m}(\operatorname{det}(f) \otimes \sigma): \operatorname{det}(H) \otimes_{\mathcal{O}_{K}} \delta_{K}^{m} \rightarrow \operatorname{det}\left(H^{*}\right) \otimes_{\mathcal{O}_{K}} \delta_{K}^{m}
$$

Note that $\sigma: \delta_{K}^{m} \rightarrow \delta_{K}^{m}$ corresponds to a non-degenerate positive-definite $\delta_{K}^{n-1}$-valued hermitian form on $\delta_{K}^{m}$ given by $(x, y) \mapsto x^{\sigma} y$. It follows that $\alpha$ is skew-hermitian and negative-definite along $\Phi$, if and only if $(-1)^{m} \operatorname{det}(f)$ is so. This we can verify after tensoring with $\mathbb{Q}$.

Choosing an orthogonal basis $\left\{h_{i}\right\}_{i=1}^{n}$ of $H_{\mathbb{Q}}$ with respect to $F_{\mathbb{Q}}, \operatorname{det}\left(H_{\mathbb{Q}}\right)$ is spanned by $\eta=$ $h_{1} \wedge \cdots \wedge h_{n}$. It follows that $\operatorname{det}\left(f_{\mathbb{Q}}\right)(\eta)(\eta)=\zeta=\prod_{i} \zeta_{i}$, where $\zeta_{i}=F_{\mathbb{Q}}\left(h_{i}, h_{i}\right)$. Since $(H, f)$ is skew-hermitian each $\zeta_{i}$ is purely imaginary. Since $n$ is odd so is $\zeta$, which shows $\operatorname{det}(f)_{\mathbb{Q}}$ is skewhermitian. As $(H, f)$ is negative-definite along $\Phi$, we have $\operatorname{Im}\left(\phi\left(\zeta_{i}\right)\right)<0$ for all $\phi \in \Phi$. Then $\operatorname{Im}(\phi(\zeta))=(-1)^{m} \prod_{i=1}^{n} \operatorname{Im}\left(\phi\left(\zeta_{i}\right)\right)$, which shows $(-1)^{m} \operatorname{det}(f)_{\mathbb{Q}}$ is negative-definite along $\Phi$. Since $\operatorname{det}(f): \operatorname{det}(H) \rightarrow \operatorname{det}\left(H^{*}\right)$ and $\sigma: \delta_{K}^{m} \rightarrow \delta_{K}^{m}$ are non-degenerate, with values in $\delta_{K}^{-n}$ and $\delta_{K}^{n-1}$ respectively, $\alpha$ is non-degenerate and $\delta_{K}^{-1}$-valued.

The following proposition is a special case of the main theorem in the next section.

Proposition 48. Suppose $\mathcal{M}_{\Phi}^{1}(\mathbb{C}) \neq \emptyset$ and $k$ is an algebraically closed field of characteristic zero. The functor

$$
\Sigma_{k}: \operatorname{Herm}_{n}\left(\mathcal{O}_{K}\right) \otimes \mathcal{M}_{\Phi}^{1}(k) \longrightarrow \mathcal{M}_{\Phi}^{n}(k)
$$

induced by Serre's construction is an equivalence of categories. 
Proof. We can again assume $k=\mathbb{C}$ by the standard descent argument. By Proposition [36, the functor $\Sigma_{\mathbb{C}}$ is fully faithful, so we must show essential surjectivity.

We have a diagram

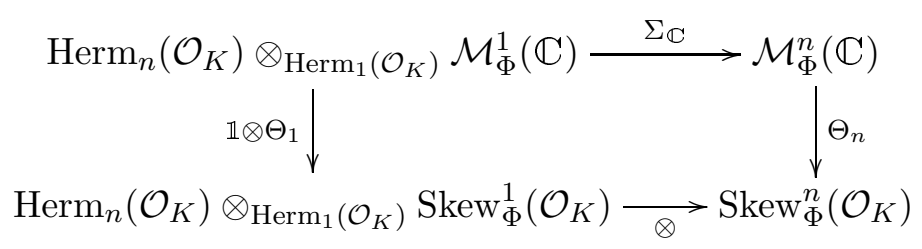

which is commutative up to isomorphism. The vertical arrows are equivalences of categories by Proposition 45. To show the top arrow is essentially surjective, it's enough to show the same for the bottom arrow. In other words, that every $(H, f) \in \operatorname{Skew}_{\Phi}^{n}\left(\mathcal{O}_{K}\right)$ is isomorphic to $\left(h \otimes \alpha, M \otimes_{\mathcal{O}_{K}} \mathfrak{a}\right)$ for some $(M, h) \in \operatorname{Herm}_{n}\left(\mathcal{O}_{K}\right)$ and $(\mathfrak{a}, \alpha) \in \operatorname{Skew}_{\Phi}^{1}\left(\mathcal{O}_{K}\right)$.

Let $(\mathfrak{a}, \alpha) \in \operatorname{Skew}_{\Phi}^{1}\left(\mathcal{O}_{K}\right)$, which is possible since $\mathcal{M}_{\Phi}^{1}(\mathbb{C}) \neq \emptyset$. We assume $\mathfrak{a}$ is a fractional ideal, identify $\mathfrak{a}^{*}$ with $\left(\mathfrak{a}^{\sigma}\right)^{-1} \delta_{K}^{-1}$, and $\alpha$ with multiplication by some $\zeta \in K$. Now let $\mathfrak{b}=\mathfrak{a}^{-1}$ and define $\beta: \mathfrak{b} \rightarrow \mathfrak{b}^{*} \cong \mathfrak{a}^{\sigma} \delta_{K}^{-1}$ by $\beta(x)=\zeta^{-1} x$. Since $(\mathfrak{a}, \alpha)$ is skew-hermitian and non-degenerate, so is $(\mathfrak{b}, \beta)$. Since $(\mathfrak{a}, \alpha)$ is $\delta_{K}^{-1}$-valued and negative-definite along $\Phi,(\mathfrak{b}, \beta)$ is $\delta_{K}$-valued and negative-definite along $\Phi \sigma$. We have $\left(\mathfrak{a} \otimes_{\mathcal{O}_{K}} \mathfrak{b}, \alpha \otimes \beta\right) \simeq\left(\mathcal{O}_{K}, \mathbb{1}\right) \in \operatorname{Herm}_{1}\left(\mathcal{O}_{K}\right)$.

For $(H, f) \in \operatorname{Skew}_{\Phi}^{n}\left(\mathcal{O}_{K}\right)$, let $(M, h)=(H, f) \otimes_{\mathcal{O}_{K}}(\mathfrak{b}, \beta)$. Since $f$ and $\beta$ are non-degenerate and skew-hermitian, with values in $\delta_{K}^{-1}$ and $\delta_{K}$ respectively, $h$ is $\mathcal{O}_{K}$-hermitian and non-degenerate. As $f$ and $\beta$ are negative definite along $\Phi$ and $\Phi \sigma$ respectively, $h$ is positive-definite. Thus $(M, h) \in$ $\operatorname{Herm}_{n}\left(\mathcal{O}_{K}\right)$. Now the bottom arrow of the diagram above takes the object $(M, h) \otimes(\mathfrak{a}, \alpha)$ to $(M, h) \otimes_{\mathcal{O}_{K}}(\mathfrak{a}, \alpha)$ which is evidently isomorphic to $(H, f)$. This shows the bottom arrow is essentially surjective, which was enough to prove the proposition.

Now let $k$ be a finite extension of the reflex field $L$, and $S$ a connected locally noetherian scheme over $\operatorname{Spec}(k)$. Suppose $(A, \iota, \lambda) \in \mathcal{M}_{\Phi}^{n}(S)$.

Proposition 49. For any $s \in S$, there exists an étale neighbourhood $U$ of s such that the triple $\left(A_{U}, \iota_{U}, \lambda_{U}\right) \in \mathcal{M}_{\Phi}^{n}(U)$, obtained from $(A, \iota, \lambda)$ by base change, arises from the Serre construction. In other words $\left(A_{U}, \iota_{U}, \lambda_{U}\right)$ lies in the essential image of the functor

$$
\Sigma_{S}: \operatorname{Herm}_{n}\left(\mathcal{O}_{K}\right) \otimes \mathcal{M}_{\Phi}^{1}(U) \longrightarrow \mathcal{M}_{\Phi}^{n}(U)
$$

Proof. Let $\mathcal{M}_{k}=\mathcal{M}_{\Phi}^{n} \otimes k \rightarrow \operatorname{Spec}(k)$ be the Deligne-Mumford stack over $\operatorname{Spec}(k)$ obtained by base change. Since $\mathcal{M}_{\Phi}^{n} \rightarrow \operatorname{Spec} \mathcal{O}_{L}$ is étale and proper by Proposition 35, so is $\mathcal{M}_{k} \rightarrow \operatorname{Spec}(k)$. Let $M \rightarrow \mathcal{M}_{k}$ be a surjective étale morphism from a scheme $M$ to $\mathcal{M}_{k}$. Then the composition $M \rightarrow \operatorname{Spec}(k)$ is an étale morphism of schemes, so $M$ is isomorphic to a disjoint union $\coprod_{\alpha} \operatorname{Spec}\left(k_{\alpha}\right)$, with each $k_{\alpha}$ a finite (separable) extension of $k$.

Let $S \rightarrow \mathcal{M}_{\Phi}^{n}$ be the morphism corresponding to the triple $(A, \iota, \lambda) \in \mathcal{M}_{\Phi}^{n}(S)$. It lifts uniquely to a morphism $S \rightarrow \mathcal{M}_{k}$, since $S \rightarrow \operatorname{Spec}\left(\mathcal{O}_{L}\right)$ factors through $\operatorname{Spec}(k) \rightarrow \operatorname{Spec}\left(\mathcal{O}_{L}\right)$. Let $S^{\prime}=$ $S \times \mathcal{M}_{k} M$ and consider the morphism $S^{\prime} \rightarrow M$ lying over $S \rightarrow \mathcal{M}_{k}$. Since $M \rightarrow \mathcal{M}_{k}$ is étale and surjective, so is $S^{\prime} \rightarrow S$.

Let $s \in S$ be a point. Let $U$ be a connected component of $S^{\prime}$ containing a point $u$ mapping to $s \in S$, so that $(U, u)$ is an étale neighbourhood of $s$. The base change triple $\left(A_{U}, \iota_{U}, \lambda_{U}\right)$ corresponds to a morphism $U \rightarrow \mathcal{M}_{\Phi}^{n}$ which factors as a composition $U \rightarrow M \rightarrow \mathcal{M}_{\Phi}^{n}$. Since $U$ is connected and $M$ is a disjoint union of $\operatorname{Spec}\left(k_{\alpha}\right)$, the map $U \rightarrow M$ further factors through some $\operatorname{Spec}\left(k^{\prime}\right) \hookrightarrow M$, where $k^{\prime}$ is a finite extension of $k$. Thus, $U \rightarrow \mathcal{M}_{\Phi}$ can be written as a composition $U \rightarrow \operatorname{Spec} k^{\prime} \rightarrow \mathcal{M}_{\Phi}^{n}$. That means there exists some triple $\left(A_{k^{\prime}}, \iota_{k^{\prime}}, \lambda_{k^{\prime}}\right)$ such that $\left(A_{U}, \iota_{U}, \lambda_{U}\right)$ is the constant triple obtained from it by base change through $U \rightarrow \operatorname{Spec}\left(k^{\prime}\right)$. Now, by Proposition 48, the triple $\left(A_{k^{\prime}}, \iota_{k^{\prime}}, \lambda_{k^{\prime}}\right)$ can be obtained by Serre's construction, after passing to a finite extension $k^{\prime \prime}$ of $k^{\prime}$. By replacing the étale neighbourhood $(U, u)$ of $s$ with a smaller one, we can assume $k^{\prime \prime}=k^{\prime}$. 
Then $\left(A_{k^{\prime}}, \iota_{k^{\prime}}, \lambda_{k^{\prime}}\right) \simeq(M, h) \otimes\left(A_{0}, \iota_{0}, \lambda_{0}\right)$ for some $(M, h) \in \operatorname{Herm}_{n}\left(\mathcal{O}_{K}\right),\left(A_{0}, \iota_{0}, \lambda_{0}\right) \in \mathcal{M}_{\Phi}^{1}\left(k^{\prime}\right)$. Therefore $\left(A_{U}, \iota_{U}, \lambda_{U}\right) \simeq(M, h) \otimes\left(A_{0 U}, \iota_{0 U}, \lambda_{0 U}\right)$, where $\left(A_{0 U}, \iota_{0 U}, \lambda_{0 U}\right) \in \mathcal{M}_{\Phi}^{1}(U)$ is the triple obtained from $\left(A_{0}, \iota_{0}, \lambda_{0}\right)$ by base change along $U \rightarrow \operatorname{Spec}\left(k^{\prime}\right)$.

Proposition 49 says if $S$ is locally noetherian over a finite extension of $L$, each triple $(A, \iota, \lambda) \in$ $\mathcal{M}_{\Phi}^{n}(S)$ can be obtained étale locally on the base $S$ by Serre's construction. In the next section this is generalized to any locally noetherian $S$ over $\operatorname{Spec} \mathcal{O}_{L}$, and interpreted in terms of stacks on the big étale site $\left(\mathrm{Sch} / \mathcal{O}_{L}\right)$ ét .

3.4. Stackification. We assume that $\mathcal{M}_{\Phi}^{1}(\mathbb{C})$ is non-empty. In Theorem 46 we showed this is almost always the case. In particular, $\mathcal{M}_{\Phi}^{1}\left(\operatorname{Spec} \mathcal{O}_{L_{0}}\right)$ is non-empty for some number field $L_{0}$.

Recall some of the constructions from $\S 2$. The 2-group $\operatorname{Herm}_{1}\left(\mathcal{O}_{K}\right)$ acts on $\operatorname{Herm}_{n}\left(\mathcal{O}_{K}\right)$ via ordinary tensor product. It also acts fibrewise on $\mathcal{M}_{\Phi}^{1}$ (Definition 25), as a category fibred in groupoids over $\operatorname{Sch} / \mathcal{O}_{L}$. Put $\mathcal{T}=\operatorname{Herm}_{n}\left(\mathcal{O}_{K}\right) \otimes_{\operatorname{Herm}_{1}\left(\mathcal{O}_{K}\right)} \mathcal{M}_{\Phi}^{1}$ in the notation of $\S 2$. We then have a functor $\mathcal{T} \rightarrow \mathrm{Sch}_{/ \mathcal{O}_{L}}$ coming from the $\mathcal{M}_{\Phi}^{1}$ factor.

Lemma 50. The category $\mathcal{T}$ is fibred in groupoids over $\mathrm{Sch} / \mathcal{O}_{L}$.

Proof. We apply Proposition 29 to deduce this, for which we need to show the action of $\operatorname{Herm}_{1}\left(\mathcal{O}_{K}\right)$ on $\mathcal{M}_{\Phi}^{1}$ is free on objects (Definition 26), and that $\mathcal{T}$ is left-cancellative over $\mathrm{Sch}_{\mathcal{O}_{L}}$ (Definition 27).

For $(\mathfrak{a}, \alpha)$ and $(\mathfrak{b}, \beta)$ in $\operatorname{Herm}_{1}\left(\mathcal{O}_{K}\right)$ and $(A, \iota, \lambda) \in \mathcal{M}_{\Phi}^{1}(S)$, suppose $(\mathfrak{a}, \alpha) \otimes(A, \iota, \lambda)$ is isomorphic to $(\mathfrak{b}, \beta) \otimes(A, \iota, \lambda)$. We claim $(\mathfrak{a}, \alpha) \simeq(\mathfrak{b}, \beta)$. By considering $\mathfrak{a} \otimes_{\mathcal{O}_{K}} A \simeq \mathfrak{b} \otimes_{\mathcal{O}_{K}} A$ on $A$-valued points we get $\mathfrak{a} \simeq \mathfrak{b}$, so without loss we can assume $\mathfrak{a}=\mathfrak{b}$. An isomorphism $(\mathfrak{a}, \alpha) \otimes(A, \iota, \lambda) \simeq$ $(\mathfrak{a}, \beta) \otimes(A, \iota, \lambda)$ is in particular an $\mathcal{O}_{K}$-linear automorphism of the abelian scheme $\mathfrak{a} \otimes_{\mathcal{O}_{K}} A$, hence of the form $\mathbb{1}_{\mathfrak{a}} \otimes \iota(r)$ for some $r \in \mathcal{O}_{K}{ }^{\times}$. To be an isomorphism of triples it must satisfy

$\alpha \otimes \lambda=\left(\mathbb{1}_{\mathfrak{a}} \otimes \iota(r)\right)^{\vee} \circ(\beta \otimes \lambda) \circ\left(\mathbb{1}_{\mathfrak{a}} \otimes \iota(r)\right)=(\beta \otimes \lambda) \circ\left(\mathbb{1} \otimes \iota\left(r^{\sigma} r\right)=\beta \otimes\left(\lambda \circ \iota\left(r^{\sigma} r\right)\right)=\left(r^{\sigma} r \cdot \beta\right) \otimes \lambda\right.$, which implies $r^{\sigma} r \cdot \beta=\alpha$. In that case the map $\mu_{r}: \mathfrak{a} \rightarrow \mathfrak{a}, x \mapsto r x$ gives an isomorphism $(\mathfrak{a}, \alpha) \simeq(\mathfrak{a}, \beta)$. This shows the action of $\operatorname{Herm}_{1}\left(\mathcal{O}_{K}\right)$ on $\mathcal{M}_{\Phi}^{1}$ is free on objects.

To show $\mathcal{T}$ is left-cancellative with respect to $\operatorname{Sch}_{/ \mathcal{O}_{L}}$, consider the functor $\mathcal{T} \rightarrow \mathcal{M}_{\Phi}^{n}$ over Sch $/ \mathcal{O}_{L}$ induced by the Serre tensor construction, which over the fibres $\mathcal{T}_{S}$ coincides with $\Sigma_{S}$. We first show this functor is faithful. By Proposition 36 it is fully faithful on each fibre. Now suppose $\alpha: T \rightarrow S$ is a morphism in $\operatorname{Sch} / \mathcal{O}_{L}$, and $\xi_{1}, \xi_{2}$ are maps $(M, h) \otimes(A, \iota, \lambda) \rightarrow(N, k) \otimes(B, \jmath, \mu)$ in $\mathcal{T}$ lying over $\alpha$, which are mapped to the same morphism in $\mathrm{Sch} / \mathcal{O}_{L}$. Any such map factors through $\gamma=\mathbb{1}_{(N, k)} \otimes p_{T}:(N, k) \otimes\left(B_{T}, \jmath_{T}, \mu_{T}\right) \rightarrow(N, k) \otimes(B, \jmath, \mu)$, where $p_{T}:\left(B_{T}, \jmath_{T}, \mu_{T}\right) \rightarrow(B, \jmath, \mu)$ is the base change map in $\mathcal{M}_{\Phi}^{1}$. Then $\xi_{1}=\gamma \circ \eta_{1}, \xi_{2}=\gamma \circ \eta_{2}$, for morphisms $\eta_{1}, \eta_{2}$ lying over $\mathbb{1}_{T}$. The image of $\mathbb{1}_{(N, k)} \otimes p_{T}$ under $\mathcal{T} \rightarrow \mathcal{M}_{\Phi}^{n}$ is the base change map in $\mathcal{M}_{\Phi}^{n}$, so it is in particular strongly cartesian. That implies $\Sigma_{T}\left(\eta_{1}\right)=\Sigma_{T}\left(\eta_{2}\right)$, which implies $\eta_{1}=\eta_{2}$, since $\Sigma_{T}$ is faithful. This shows $\mathcal{T} \rightarrow \mathcal{M}_{\Phi}^{n}$ is also faithful. Now, since $\mathcal{M}_{\Phi}^{n}$ is fibred in groupoids over $\mathrm{Sch}_{/ \mathcal{O}_{L}}$, by Lemma 28 it is left-cancellative over $\mathrm{Sch}_{/ \mathcal{O}_{L}}$. Then $\mathcal{T}$ is also left-cancellative over $\mathrm{Sch} / \mathcal{O}_{L}$, by faithfulness of $\mathcal{T} \rightarrow \mathcal{M}_{\Phi}^{n}$. Thus by Proposition 29 , $\mathcal{T}$ is also fibred in groupoids over $\mathrm{Sch} / \mathcal{O}_{L}$.

By Proposition [36, for each $S \in \operatorname{Sch}_{\mathcal{O}_{L}}$ the functor $\Sigma_{S}: \mathcal{T}(S) \rightarrow \mathcal{M}_{\Phi}^{n}(S)$ is fully faithful. Since $\mathcal{T}$ is fibred in groupoids over $\mathrm{Sch}_{/ \mathcal{O}_{L}}$, the morphism $\mathcal{T} \rightarrow \mathcal{M}_{\Phi}^{n}$ induced by the Serre construction is also fully faithful.

We define $\operatorname{Herm}_{n}\left(\mathcal{O}_{K}\right) \otimes \mathcal{M}_{\Phi}^{1}$ to be the stack associated to the presheaf $\mathcal{T}$ on the big étale site over $\operatorname{Spec} \mathcal{O}_{L}$. Here we have suppressed the subscript $\operatorname{Herm}_{1}\left(\mathcal{O}_{K}\right)$ from the notation to differentiate it from $\mathcal{T}$. Since the functor $\mathcal{T} \rightarrow \mathcal{M}_{\Phi}^{n}$ is fully faithful and $\mathcal{M}_{\Phi}^{n}$ is a stack, the presheaf $\mathcal{T}$ is already separated. It follows that $\operatorname{Herm}_{n}\left(\mathcal{O}_{K}\right) \otimes \mathcal{M}_{\Phi}^{1}$ is obtained from $\mathcal{T}$ by one application of the plus construction. In other words, $\left(\operatorname{Herm}_{n}\left(\mathcal{O}_{K}\right) \otimes \mathcal{M}_{\Phi}^{1}\right)(S)$ consists of descent data relative to étale coverings of $S$, with the appropriate morphisms [15, Ch. 3, Lemma 3.2]. 
We thus obtain a commutative diagram of categories fibred in groupoids

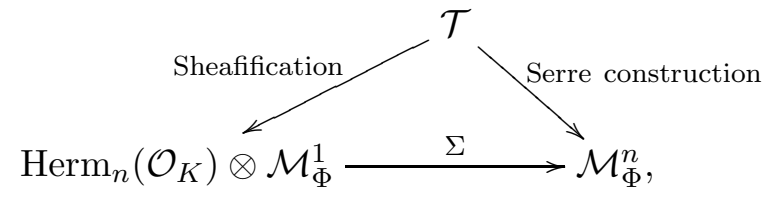

where $\Sigma$ is the functor induced by the universal property of sheafication.

Our results so far can be summarized as follows: the functor $\Sigma$ in the diagram $(\Delta)$ identifies $\operatorname{Herm}_{n}\left(\mathcal{O}_{K}\right) \otimes \mathcal{M}_{\Phi}^{n}$ with a full subcategory of $\mathcal{M}_{\Phi}^{n}$. Furthermore, when $S$ is locally noetherian over a finite extension of $L$, the induced functor $\left(\operatorname{Herm}_{n}\left(\mathcal{O}_{K}\right) \otimes \mathcal{M}_{\Phi}^{n}\right)(S) \rightarrow \mathcal{M}_{\Phi}^{n}(S)$ is an equivalence of categories, by Proposition 49. The fact that $\mathcal{M}_{\Phi}^{n}$ is étale over $\operatorname{Spec} \mathcal{O}_{L}$ allows us to extend this to characteristic $p$.

Proposition 51. Let $S=\operatorname{Spec}(k)$ for $k$ a perfect field of characteristic $p$. The functor $\Sigma$ in $(\triangle)$ induces an equivalence of categories on the fibre over $S$.

Proof. Let $W_{k}$ be the ring of Witt vectors of $k$, with fraction field $W_{k}\left[p^{-1}\right]$. Since $\mathcal{M}_{\Phi}^{n}$ is smooth, an object $\left(A_{0}, \iota_{0}, \lambda_{0}\right) \in \mathcal{M}_{\Phi}^{n}(k)$ lifts to $(A, \iota, \lambda) \in \mathcal{M}_{\Phi}^{n}\left(W_{k}\right)$. By Proposition 48, after base changing to a finite extension $F$ of $W_{k}\left[p^{-1}\right]$, we have an isomorphism $\left(A_{F}, \iota_{F}, \lambda_{F}\right) \simeq(M, h) \otimes(B, \jmath, \mu)$ for some $(B, \jmath, \mu) \in \mathcal{M}_{\Phi}^{1}(F)$, and $(M, h) \in \operatorname{Herm}_{n}\left(\mathcal{O}_{K}\right)$. Since $B$ has CM, after possibly enlarging $F$ again it has good reduction, and its Néron model $\mathscr{B}$ over $\mathcal{O}_{F}$ is an abelian scheme [25].

By the Néron mapping property, the action of $\mathcal{O}_{K}$ and the polarization $\mu$ also lift to $\mathscr{B}$, so we have a triple $\left(\mathscr{B}, \jmath_{\mathscr{B}}, \mu_{\mathscr{B}}\right) \in \mathcal{M}_{\Phi}^{1}\left(\mathcal{O}_{F}\right)$, and we can form $(M, h) \otimes_{\mathcal{O}_{K}}\left(\mathscr{B}, \jmath_{\mathscr{B}}, \mu_{\mathscr{B}}\right) \in \mathcal{M}_{\Phi}^{n}\left(\mathcal{O}_{F}\right)$. Now it is easy to verify that Serre's construction commutes with taking Néron models, so $M \otimes_{\mathcal{O}_{K}} \mathscr{B}$ is the Néron model of $M \otimes_{\mathcal{O}_{K}} B$. On the other hand, the base change $\mathscr{A}$ of $A$ to $\operatorname{Spec} \mathcal{O}_{F}$ is also a Néron model for its generic fibre, which is again $M \otimes_{\mathcal{O}_{K}} B$, so we have $\mathscr{A} \simeq M \otimes_{\mathcal{O}_{K}} \mathscr{B}$ by uniqueness of the model, which implies $\left(\mathscr{A}, \iota_{\mathcal{O}_{F}}, \lambda_{\mathcal{O}_{F}}\right) \simeq(M, h) \otimes\left(\mathscr{B}, \jmath_{\mathscr{B}}, \mu_{\mathscr{B}}\right)$ by the mapping property.

Let $k^{\prime}$ denote the residue field of $\mathcal{O}_{F}$, a finite extension of $k$. The isomorphism $\left(\mathscr{A}, \iota_{\mathcal{O}_{F}}, \lambda_{\mathcal{O}_{F}}\right) \simeq$ $(M, h) \otimes(\mathscr{B}, \jmath, \mu)$ reduces modulo the prime of $\mathcal{O}_{F}$ to an isomorphism $\left(A^{\prime}, \iota^{\prime}, \lambda^{\prime}\right) \simeq(M, h) \otimes\left(B^{\prime}, \jmath^{\prime}, \mu^{\prime}\right)$ over $k^{\prime}$. Since $\left(A^{\prime}, \iota^{\prime}, \lambda^{\prime}\right)$ is the base change of $\left(A_{0}, \iota_{0}, \lambda_{0}\right)$ to $k^{\prime}$, we have shown that an arbitrary triple $\left(A_{0}, \iota_{0}, \lambda_{0}\right) \in \mathcal{M}_{\Phi}^{n}(k)$ arises from Serre's construction after passing to an étale cover $\operatorname{Spec}\left(k^{\prime}\right) \rightarrow$ $\operatorname{Spec}(k)$. Thus on the fibre over $\operatorname{Spec}(k)$ the functor $\Sigma$ is essentially surjective, and being fully faithful by Proposition 36, is an equivalence of categories.

We can now prove the main theorem.

Theorem 52. If $\mathcal{M}_{\Phi}^{1}(\mathbb{C}) \neq \emptyset$, the functor $\Sigma: \operatorname{Herm}_{n}\left(\mathcal{O}_{K}\right) \otimes \mathcal{M}_{\Phi}^{1} \rightarrow \mathcal{M}_{\Phi}^{n}$ is an isomorphism of stacks.

Proof. We will prove this by showing that $\Sigma$ induces equivalences of categories on the stalks of the geometric points of the big étale site on $\operatorname{Spec}\left(\mathcal{O}_{L}\right)$. By definition these are the geometric points $\bar{s} \rightarrow S$, where $S$ is a scheme locally of finite type over $\operatorname{Spec}\left(\mathcal{O}_{L}\right)$. Since $\mathcal{O}_{L}$ is a Jacobson ring, it's enough to only consider geometric points $\bar{s} \rightarrow S$ having image $s \in S$ lying over a closed point $t \in \operatorname{Spec}\left(\mathcal{O}_{L}\right)$ [19, II, Remark 2.17(b)]. In that case since $S$ is locally of finite type, $s \in S$ is closed and $k(t) \subset k(s)$ is a finite extension. As $k(t)$ is a finite field, $k(s)$ is perfect, and in particular Proposition 51 applies to $k=k(s)$.

Let $(A, \iota, \lambda) \in \mathcal{M}_{\Phi}^{n}(S)$ be a triple corresponding to a morphism $S \rightarrow \mathcal{M}_{\Phi}^{n}$ and suppose $\bar{s} \rightarrow S$ is a geometric point whose image $s \in S$ is closed, with $k(s)$ perfect. We want to show there exists a finite étale morphism $U \rightarrow S$ through which $\bar{s} \rightarrow S$ factors, such that the base change $\left(A_{U}, \iota_{U}, \lambda_{U}\right)$ of $(A, \iota, \lambda)$ to $U$ is obtained by the Serre construction.

Let $\left(A_{s}, \iota_{s}, \lambda_{s}\right) \in \mathcal{M}_{\Phi}^{n}(k(s))$ denote the fibre of $(A, \iota, \lambda)$ over $s$, which corresponds to the composition Spec $k(s) \rightarrow S \rightarrow \mathcal{M}_{\Phi}^{n}$. By Proposition 51 there exists a finite extension $k^{\prime}$ of $k(s)$ contained 
in $k(\bar{s})$, such that the base change $\left(A_{s^{\prime}}, \iota_{s^{\prime}}, \lambda_{s^{\prime}}\right)$ of $\left(A_{s}, \iota_{s}, \lambda_{s}\right)$ to $s^{\prime}=\operatorname{Spec}\left(k^{\prime}\right)$ is isomorphic to $(M, h) \otimes\left(B_{0}, \jmath_{0}, \mu_{0}\right)$ for some $(M, h) \in \operatorname{Herm}_{n}\left(\mathcal{O}_{K}\right)$ and $\left(B_{0}, \jmath_{0}, \mu_{0}\right) \in \mathcal{M}_{\Phi}^{1}\left(k\left(s^{\prime}\right)\right)$.

Now, let $\mathcal{S}_{(M, h)}: \mathcal{M}_{\Phi}^{1} \rightarrow \mathcal{M}_{\Phi}^{n}$ be the morphism of stacks over $\operatorname{Spec}\left(\mathcal{O}_{L}\right)$ given on sections by $(B, \jmath, \mu) \mapsto(M, h) \otimes(B, \jmath, \mu)$. Let $S^{\prime}=S \times_{\mathcal{M}_{\Phi}^{n}} \mathcal{M}_{\Phi}^{1}$. We have a commutative diagram,

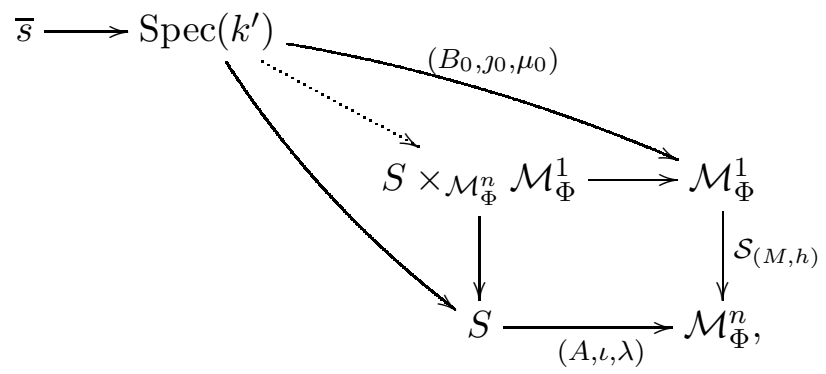

where $(A, \iota, \lambda)$ and $\left(B_{0}, \jmath_{0}, \mu_{0}\right)$ label their corresponding morphisms into $\mathcal{M}_{\Phi}^{n}$ and $\mathcal{M}_{\Phi}^{1}$, respectively.

By the universal property of the fibre product, for any morphism $U \rightarrow S$, the base change triple $\left(A_{U}, \iota_{U}, \lambda_{U}\right)$ arises from Serre's construction with $(M, h)$, if and only if the map $U \rightarrow S$ factors through $S^{\prime} \rightarrow S$. Thus $\operatorname{Spec}\left(k^{\prime}\right) \rightarrow S$ factors as in the diagram, since $\left(A_{s^{\prime}}, \iota_{s^{\prime}}, \lambda_{s^{\prime}}\right) \simeq$ $(M, h) \otimes\left(B_{0}, \jmath_{0}, \mu_{0}\right)$. We wish to find a finite étale morphism of schemes $U \rightarrow S$, which factors through $S^{\prime} \rightarrow S$, and through which $\bar{s} \rightarrow S$ factors. The former condition means that $\left(A_{U}, \iota_{U}, \lambda_{U}\right)$ arises from Serre's construction with $(M, h)$, and the latter that $U \rightarrow S$ is an étale neighbourhood of $\bar{s} \rightarrow S$. We claim that $S^{\prime} \rightarrow S$ itself is an étale morphism of schemes, so that we can take $U=S^{\prime}$.

We first note that $\mathcal{S}_{(M, h)}: \mathcal{M}_{\Phi}^{1} \rightarrow \mathcal{M}_{\Phi}^{n}$ is étale, proper, and representable by algebraic spaces. Indeed, by Theorem [35] the stacks $\mathcal{M}_{\Phi}^{n}$ and $\mathcal{M}_{\Phi}^{1}$ are étale and proper $\operatorname{over} \operatorname{Spec}\left(\mathcal{O}_{L}\right)$, so any $\mathcal{O}_{L^{-}}$ morphism $\mathcal{M}_{\Phi}^{n} \rightarrow \mathcal{M}_{\Phi}^{1}$ is étale and proper. The morphism $\mathcal{S}_{(M, h)}$ is also representable by algebraic spaces, because tensoring with $(M, h)$ is a faithful functor $\mathcal{M}_{\Phi}^{1}(T) \rightarrow \mathcal{M}_{\Phi}^{n}(T)$ for any $T \in\left(\operatorname{Sch}_{/ \mathcal{O}_{L}}\right)$. It follows that $S^{\prime} \rightarrow S$ is an étale and proper morphism of algebraic spaces. Now any separated and locally quasi-finite morphism of algebraic spaces, in particular $S^{\prime} \rightarrow S$, is representable by schemes. Then since $S$ itself is a scheme, so is $S^{\prime}$.

Then if $U=S^{\prime}$, and $u$ is the composition $\bar{s} \rightarrow \operatorname{Spec}\left(k^{\prime}\right) \rightarrow S^{\prime}$, we have the desired étale neighbourhood $(u, U)$ of $\bar{s} \rightarrow S$. In other words, we have shown that for every triple $(A, \iota, \lambda) \in$ $\mathcal{M}_{\Phi}^{n}(S)$, and every geometric point $\bar{s} \rightarrow S$ whose image $s \in S$ is closed, there exists an étale neighbourhood of $\bar{s} \rightarrow S$ such that the triple $\left(A_{U}, \iota_{U}, \lambda_{U}\right)$ obtained by base change is in the essential image of $\Sigma_{U}$. Hence $\Sigma$ induces an essentially surjective functor $\Sigma_{\bar{s} / S}$ on the étale stalks at $\bar{s} \rightarrow S$. Since $\Sigma$ is fully faithful by Proposition $36, \Sigma_{\bar{s} / S}$ is an equivalence of categories. As the points $\bar{s} \rightarrow S$ form a very dense subset of all geometric points of the big étale site over Spec $\mathcal{O}_{L}$, it follows that $\Sigma$ is an isomorphism of étale sheaves, hence an isomorphism of stacks.

To prevent language from obscuring content, we restate the results in plainer terms below.

Theorem 53. Let $S$ be a connected locally noetherian scheme over $\operatorname{Spec} \mathcal{O}_{L}$.

(a) For every object $(A, \iota, \lambda)$ of $\mathcal{M}_{\Phi}^{n}(S)$, and every point $s \in S$, there exists an étale neighbourhood $U \rightarrow S$ of $s$, as well as objects $(M, h) \in \operatorname{Herm}_{n}\left(\mathcal{O}_{K}\right),\left(A_{0}, \iota_{0}, \lambda_{0}\right) \in \mathcal{M}_{\Phi}^{1}(U)$, such that there is an isomorphism of triples

$$
\left(A_{U}, \iota_{U}, \lambda_{U}\right) \stackrel{\sim}{\longrightarrow}(M, h) \otimes_{\mathcal{O}_{K}}\left(A_{0}, \iota_{0}, \lambda_{0}\right) .
$$

(b) For a morphism $\phi:(A, \iota, \lambda) \rightarrow(B, \jmath, \mu)$ of $\mathcal{M}_{\Phi}^{n}(S)$, let $\left\{U_{i} \rightarrow S\right\}_{i \in I}$ be a cover of $S$ by étale morphisms such that, as in (a), there are isomorphisms

$$
\psi_{i}:\left(A_{U_{i}}, \iota_{U_{i}}, \lambda_{U_{i}}\right) \stackrel{\sim}{\longrightarrow}\left(M_{i}, h_{i}\right) \otimes\left(A_{i}, \iota_{i}, \lambda_{i}\right), \quad \psi_{i}^{\prime}:\left(B_{U_{i}}, \jmath_{U_{i}}, \mu_{U_{i}}\right) \stackrel{\sim}{\longrightarrow}\left(N_{i}, k_{i}\right) \otimes\left(B_{i}, \jmath_{i}, \lambda_{i}\right) .
$$


Then there exist $\left(\mathfrak{a}_{i}, \alpha_{i}\right) \in \operatorname{Herm}_{1}\left(\mathcal{O}_{K}\right)$, where $\mathfrak{a}_{i}=\operatorname{Hom}_{\mathcal{O}_{K}}\left(A_{i}, B_{i}\right)$, and isomorphisms

$$
f_{i}:\left(M_{i}, h_{i}\right) \stackrel{\sim}{\longrightarrow}\left(N_{i}, k_{i}\right) \otimes_{\mathcal{O}_{K}}\left(\mathfrak{a}_{i}, \alpha_{i}\right), \quad \phi_{i}:\left(A_{i}, \iota_{i}, \lambda_{i}\right) \stackrel{\sim}{\longrightarrow}\left(\mathfrak{a}_{i}^{\vee}, \alpha_{i}^{\vee}\right) \otimes\left(B_{i}, J_{i}, \mu_{i}\right),
$$

such that $\psi_{i}^{-1} \circ \phi \circ \psi_{i}=\omega_{i} \circ\left(f_{i} \otimes \phi_{i}\right)$ for each $i \in I$, and $\omega_{i}$ is a canonical isomorphism

$$
\left(\left(N_{i}, k_{i}\right) \otimes \mathcal{O}_{K}\left(\mathfrak{a}_{i}, \alpha_{i}\right)\right) \otimes\left(\left(\mathfrak{a}_{i}^{\vee}, \alpha_{i}^{\vee}\right) \otimes\left(A_{i}, \iota_{i}, \lambda_{i}\right)\right) \stackrel{\sim}{\longrightarrow}\left(N_{i}, k_{i}\right) \otimes\left(A_{i}, \iota_{i}, \lambda_{i}\right) .
$$

3.5. A simple example. Let $(K, \Phi)$ consist of a quadratic imaginary extension $K / \mathbb{Q}$ and an embedding $K \subset \mathbb{C}$. Let $H$ be the Hilbert class field of $K$, and $E$ an elliptic curve with CM by $\mathcal{O}_{K}$, defined over $H$. Let $F$ be a quadratic extension of $H$, and put $A=\operatorname{Res}_{H}^{F} E_{F}$. Then $A_{F} \simeq E_{F} \times E_{F} \simeq \mathcal{O}_{K}^{2} \otimes_{\mathcal{O}_{K}} E_{F}$. We claim $A$ itself is not isomorphic to any $M \otimes_{\mathcal{O}_{K}} E^{\prime}$ over $H$.

Assume $A \simeq M \otimes_{\mathcal{O}_{K}} E^{\prime}$. Since $E^{\prime}$ also has $\mathrm{CM}$ by $\mathcal{O}_{K}, E^{\prime} \simeq E^{\tau}$ for some $\tau \in \operatorname{Gal}(H / K)$. Then $E^{\prime} \simeq \mathfrak{a} \otimes \mathcal{O}_{K} E$ for some fractional ideal $\mathfrak{a}$ by the main theorem of complex multiplication. Hence $A \simeq M_{\mathfrak{a}} \otimes_{\mathcal{O}_{K}} E$ with $M_{\mathfrak{a}}=M \otimes_{\mathcal{O}_{K}} \mathfrak{a}$, so $A_{F} \simeq M_{\mathfrak{a}} \otimes_{\mathcal{O}_{K}} E_{F}$. On the other hand $A_{F} \simeq \mathcal{O}_{K}^{2} \otimes_{\mathcal{O}_{K}} E_{F}$, so $M_{\mathfrak{a}} \simeq \mathcal{O}_{K}^{2}$ and $A \simeq E^{2}$. This is a contradiction, since $\operatorname{Res}_{H}^{F} E_{F}$ is not isomorphic to $E \times E$.

Now fix $\jmath$ and the canonical $\mu$ such that $(E, \jmath, \mu) \in \mathcal{M}_{\Phi}^{1}(H)$. The product polarization $\mu_{F} \times$ $\mu_{F}$ commutes with the automorphism of $E_{F} \times E_{F}$ that switches the factors, so it descends to a polarization $\lambda$ on $A$. Likewise, the $\mathcal{O}_{K}$-action on $E_{F} \times E_{F}$ descends to an $\iota$ on $A$, and we have $(A, \iota, \lambda) \in \mathcal{M}_{\Phi}^{2}(H)$. We saw that $(A, \iota, \lambda)$ is not given by the Serre construction. However, the base change $\left(A_{F}, \iota_{F}, \lambda_{F}\right)$ is isomorphic to $\left(\mathcal{O}_{K}^{2}, h\right) \otimes\left(E_{F}, \jmath_{F}, \mu_{F}\right)$, where $h: \mathcal{O}_{K}^{2} \rightarrow\left(\mathcal{O}_{K}^{2}\right)^{\vee}$ corresponds to $H\left(\left(x_{1}, x_{2}\right),\left(y_{1}, y_{2}\right)\right)=x_{1}^{\sigma} y_{1}+x_{2}^{\sigma} y_{2}$.

Then $(A, \iota, \lambda) \in \mathcal{M}_{\Phi}^{2}(H)$ arises from the Serre construction only after passing to the étale cover Spec $F \rightarrow$ Spec $H$. Equivalently, $A$ is obtained by gluing two copies of $\mathcal{O}_{K}^{2} \otimes_{\mathcal{O}_{K}} E$ non-trivially along the self-intersection of the étale neighbourhood $\operatorname{Spec} F \rightarrow \operatorname{Spec} H$. The corresponding automorphism is given by $(x, y) \mapsto(y, x)$ on the $\mathcal{O}_{K}^{2}$ factor. Since that is not induced by an automorphism of $E$, the same gluing can not happen in $\mathcal{M}_{\Phi}^{1}(H)$. Thus sheafifying $S \mapsto \operatorname{Herm}_{n}\left(\mathcal{O}_{K}\right) \otimes_{\mathcal{O}_{K}} \mathcal{M}_{\Phi}^{1}(S)$ accounts for objects glued together by automorphisms coming from $\operatorname{Herm}_{n}\left(\mathcal{O}_{K}\right)$.

\section{FinAL REMARKS}

The results of $\S 1$, in particular Theorem 17 and Proposition 18, are more general than the use we've made of them. They may have further applications, for instance to moduli spaces of abelian schemes with action by an order in a quaternion algebra.

Since Serre's construction commutes with base change, Proposition 48 allows a description of the action of $\operatorname{Aut}(\mathbb{C})$ on $\mathcal{M}_{\Phi}^{n}$, by relating it to the description of the action on $\mathcal{M}_{\Phi}^{1}$ given by the theory of complex multiplication [2, appx A]. We hope to further explore this in another article.

\section{REFERENCES}

[1] H. Braun and M. Koecher. Jordan-Algebren, volume 128 of Die Grundlehren Der Mathematischen Wissenschaften In Einzeldarstellungen. Springer-Verlag, 1966.

[2] Ching-Li Chai, Brian Conrad, and Frans Oort. Complex multiplication and lifting problems, volume 195 of Mathematical Surveys and Monographs. American Mathematical Society, Providence, RI, 2014.

[3] P. E. Conner and J. Hurrelbrink. Class number parity, volume 8 of Series in Pure Mathematics. World Scientific Publishing Co., Singapore, 1988.

[4] Brian Conrad. Gross-Zagier revisited. In Heegner points and Rankin L-series, volume 49 of Math. Sci. Res. Inst. Publ., pages 67-163. Cambridge Univ. Press, Cambridge, 2004. With an appendix by W. R. Mann.

[5] P. Deligne. Catégories tannakiennes. In The Grothendieck Festschrift, Vol. II, volume 87 of Progr. Math., pages 111-195. Birkhäuser Boston, Boston, MA, 1990.

[6] Gerd Faltings and Ching-Li Chai. Degeneration of abelian varieties, volume 22 of Ergebnisse der Mathematik und ihrer Grenzgebiete (3) [Results in Mathematics and Related Areas (3)]. Springer-Verlag, Berlin, 1990. With an appendix by David Mumford.

[7] Daniel Grayson. Higher algebraic K-theory. II (after Daniel Quillen). In Algebraic K-theory (Proc. Conf., Northwestern Univ., Evanston, Ill., 1976), pages 217-240. Lecture Notes in Math., Vol. 551. Springer, Berlin, 1976. 
[8] A. Grothendieck and M. Demazure. Schémas en groupes (SGA 3). Tome I. Propriétés générales des schémas en groupes. Documents Mathématiques (Paris) [Mathematical Documents (Paris)], 7. Société Mathématique de France, Paris, 2011. Séminaire de Géométrie Algébrique du Bois Marie 1962-64. [Algebraic Geometry Seminar of Bois Marie 1962-64], A seminar directed by M. Demazure and A. Grothendieck with the collaboration of M. Artin, J.-E. Bertin, P. Gabriel, M. Raynaud and J-P. Serre, Revised and annotated edition of the 1970 French original.

[9] Haruzo Hida. p-adic automorphic forms on Shimura varieties. Springer Monographs in Mathematics. SpringerVerlag, New York, 2004.

[10] Benjamin Howard. Complex multiplication cycles and Kudla-Rapoport divisors. Ann. of Math. (2), 176(2):10971171, 2012.

[11] Benjamin Howard. Complex multiplication cycles and Kudla-Rapoport divisors, II. Amer. J. Math., 137(3):639698, 2015.

[12] Stephen Kudla and Michael Rapoport. Special cycles on unitary Shimura varieties I. Unramified local theory. Invent. Math., 184(3):629-682, 2011.

[13] Stephen Kudla and Michael Rapoport. Special cycles on unitary Shimura varieties II: Global theory. J. Reine Angew. Math., 697:91-157, 2014.

[14] Kai-Wen Lan. Arithmetic compactifications of PEL-type Shimura varieties, volume 36 of London Mathematical Society Monographs Series. Princeton University Press, Princeton, NJ, 2013.

[15] Gérard Laumon and Laurent Moret-Bailly. Champs algébriques, volume 39 of Ergebnisse der Mathematik und ihrer Grenzgebiete. 3. Folge. A Series of Modern Surveys in Mathematics [Results in Mathematics and Related Areas. 3rd Series. A Series of Modern Surveys in Mathematics]. Springer-Verlag, Berlin, 2000.

[16] Kristin Lauter. The maximum or minimum number of rational points on genus three curves over finite fields. Compositio Math., 134(1):87-111, 2002. With an appendix by Jean-Pierre Serre.

[17] Saunders Mac Lane. Categories for the working mathematician, volume 5 of Graduate Texts in Mathematics. Springer-Verlag, New York, second edition, 1998.

[18] Kevin McCrimmon. A taste of Jordan algebras. Universitext. Springer-Verlag, New York, 2004.

[19] James S. Milne. Étale cohomology, volume 33 of Princeton Mathematical Series. Princeton University Press, Princeton, N.J., 1980.

[20] D. Mumford, J. Fogarty, and F. Kirwan. Geometric invariant theory, volume 34 of Ergebnisse der Mathematik und ihrer Grenzgebiete (2) [Results in Mathematics and Related Areas (2)]. Springer-Verlag, Berlin, third edition, 1994.

[21] David Mumford. Abelian varieties, volume 5 of Tata Institute of Fundamental Research Studies in Mathematics. Published for the Tata Institute of Fundamental Research, Bombay, 2008. With appendices by C. P. Ramanujam and Yuri Manin, Corrected reprint of the second (1974) edition.

[22] Georgios Pappas. On the arithmetic moduli schemes of PEL Shimura varieties. J. Algebraic Geom., 9(3):577-605, 2000.

[23] M. Rapoport. Compactifications de l'espace de modules de Hilbert-Blumenthal. Compositio Math., 36(3):255$335,1978$.

[24] Neantro Saavedra Rivano. Catégories Tannakiennes. Lecture Notes in Mathematics, Vol. 265. Springer-Verlag, Berlin, 1972.

[25] Jean-Pierre Serre and John Tate. Good reduction of abelian varieties. Ann. of Math. (2), 88:492-517, 1968.

[26] Goro Shimura. Abelian varieties with complex multiplication and modular functions, volume 46 of Princeton Mathematical Series. Princeton University Press, Princeton, NJ, 1998.

[27] D. Tambara. A duality for modules over monoidal categories of representations of semisimple Hopf algebras. J. Algebra, 241(2):515-547, 2001.

[28] Charles A. Weibel. The K-book, volume 145 of Graduate Studies in Mathematics. American Mathematical Society, Providence, RI, 2013. An introduction to algebraic $K$-theory. 